\title{
User's Guide for the PHIGs Validation Tests (Version 2)
}

\author{
John Cugini \\ Mary T. Gunn \\ Lynne S. Rosenthal
}

U.S. DEPARTMENT OF COMMERCE Technology Administration

National Institute of Standards and Technology

Computer Systems Laboratory

Gaithersburg, MD 20899 



\section{User's Guide for the PHIGS Validation Tests (Version 2)}

\author{
John Cugini \\ Mary T. Gunn \\ Lynne S. Rosenthal
}

\section{U.S. DEPARTMENT OF COMMERCE Technology Administration National Institute of Standards and Technology \\ Computer Systems Laboratory Gaithersburg, MD 20899}

October 1992

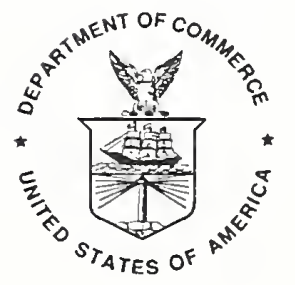

U.S. DEPARTMENT OF COMMERCE Barbara Hackman Franklin, Secretary

TECHNOLOGY ADMINISTRATION

Robert $M$. White, Under Secretary for Technology

NATIONAL INSTITUTE OF STANDARDS

AND TECHNOLOGY

John W. Lyons, Director 

USER'S GUIDE FOR THE

PHIGS VALIDATION TESTS

(Version 2)

by

John Cugini

Mary T. Gunn

Lynne S. Rosenthal

ABSTRACT: The PHIGS Validation Tests (PVT), developed by NIST, consist of a large set of Fortran programs which may be used to test how well implementations of PHIGS conform to the standard. The tests are organized into a hierarchical structure of modules which corresponds to the conceptual overview of the standard. The tests are associated with the standard via a set of semantic requirements which are derived directly from the standard. Cross-reference tables allow the user to find tests relating to specific PHIGS functions and data structures. Directions for installation and operation of the tests are included.

KEYWORDS: conformance testing; graphics standards; PHIGS; testing of software; validation of software 

CONTENTS

INTRODUCTION . . . . . . . . . . . . . . . . . . 1

2

2.1

2.2

2.3

2.4

2.5

2.5 .1

2.5 .2

2.5 .2 .1

2.5.2. 2

2.5.2.3

2.5.2. 4

2.5.2. 5

2.5 .2 .6

2.5 .3

2.5. 4

2.5 .5

2.5 .6

2.5 .6 .1

2.5 .6 .2

2.6

2.6 .1

2.6 .2

2.6 .3

2.6 .4

2.6 .5

2.6 .6

2.7

2.7 .1

2.7 .2

2.7 .3

2.7 .4

3

3.1

3.2

3. 2.1

3.2 .2

3.2 .3

3.2 .4

3. 2.5

3.2 .6

3. 2.7

3. 2.8

3.2 .9

3.3
STRUCTURE AND FORMAT OF PVT SUITE . . . . . . . . 2

Tree Structure . . . . . . . . . . . . . . . . 2

Modules . . . . . . . . . . . . . . . . . . 2

Subroutine Libraries . . . . . . . . . . . . . 3

Order . . . . . . . . . . . . . . . . . 4 4

Module Documentation . . . . . . . . . . . . 4

Introductory Headings . . . . . . . . . . . . 4

Semantic Requirements . . . . . . . . . . . . 4

Related Functions . . . . . . . . . . 6

Related Data Structures . . . . . . . . 6

Basis In The Standard . . . . . . . . 6

Test Cases... . . . . . . . . . . 7

Module Cross-references . . . . . . . . . 7

Comments . . . . . . . . . . . . . . 7

Local Dictionary . . . . . . . . . . . . . 7

Semantic Cross-references . . . . . . . . . 7

Local Subroutines . . . . . . . . . . . . 7

Program Design . . . . . . . . . . . . 8

Headings . . . . . . . . . . . . . . . 8

Logic And TCs . . . . . . . . . . . . . 9

Source Code Conventions . . . . . . . . . . . 9

Language . . . . . . . . . . . . . . 10

All Variables Declared . . . . . . . . . 10

Standard PHIGS Names . . . . . . . . . . 10

Dummy Parameters . . . . . . . . . . . 10

Program Banner . . . . . . . . . . . . 10

Common . . . . . . . . . . . . . . • • . . 11

Ubiquitous Subroutines . . . . . . . . . . 11

Initialization And Clean-up of Test Programs . 11

TC Subroutines . . . . . . . . . . . . . 11

Message Subroutines . . . . . . . . . . 12

CHKINQ . . . . . . . . . . . . . . . 12

INSTALLATION . . . . . . . . . . . . . . 13

File Hierarchy . . . . . . . . . . . . . . . 13

Customization of Code . . . . . . . . . . . 14

Naming PVT Configuration File . . . . . . . 14

Special Processing For Opening PHIGS . . . . 15

Random Number Generator . . . . . . . . 15

Naming Individual Message File... . . . . 15

Resolution of Parameters For <Open Workstation> 15 Providing Valid Names For Archive Files . . . 16

Time-stamping Message Files . . . . . . . 16

Operator Communication . . . . . . . . . 16

End of File ..... . . . . . . . . . 16

Linking Subroutines . . . . . . . . . . . 16 


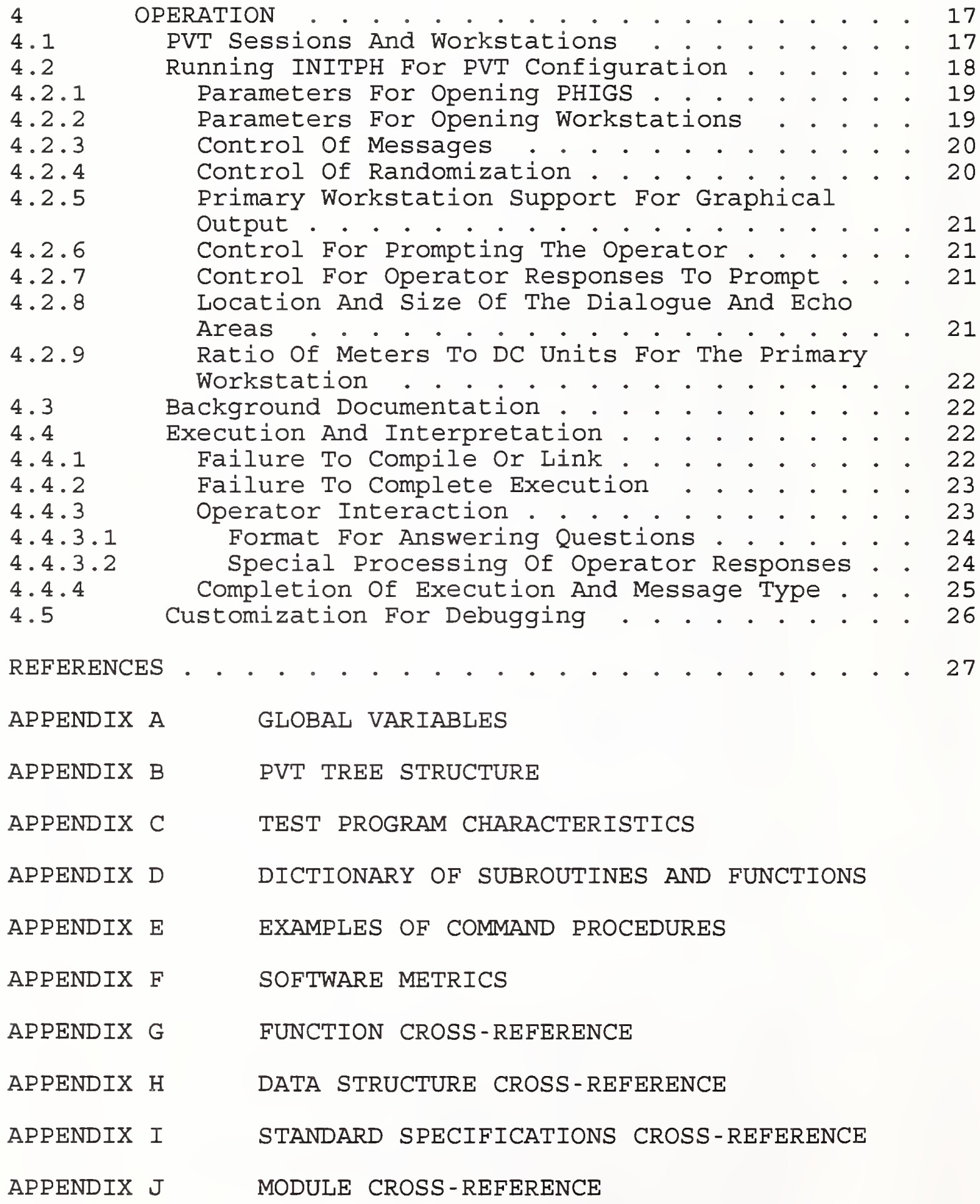


PHIGS stands for Programmer's Hierarchical Interactive Graphics System. The PHIGS standard defines a set of functions to be used by a programmer to manipulate and display 3-D graphical objects. For a full description of the features of PHIGS, see [PHIGS89]. The standard has been approved by the American National Standards Institute (ANSI) as ANSI X3.144-1988, by the International Organization for Standards (ISO) as ISO 9592-1:1989, and by the Federal government as Federal Information Processing Standard (FIPS) 153.

The PHIGS Validation Test (PVT) suite is a product of the Computer Systems Laboratory (CSL) of the National Institute of Standards and Technology (NIST). The function of this suite is to test whether implementations of PHIGS conform to the PHIGS standard. The PVT is available to individuals and organizations for use in developing or testing PHIGS implementations. CSL uses version 2 of this suite to validate PHIGS implementations which have been submitted for testing.

There are some aspects of the PHIGS standard which can reasonably be construed in various ways. The PVT suite embodies CSL's best technical judgment concerning the standard's requirements for conforming implementations. CSL intends to update the PVT suite periodically to reflect official ANSI or ISO interpretations that conflict with the assumptions upon which the current version of the PVT is based.

This document describes the general rules and procedures for using the PVT suite. Detailed information for specific tests may be found in the module documentation, as described in section 2.5. For a general discussion of PVT design issues, see [CUGI90] and [CUGI91].

We welcome any comments or suggestions regarding the PVT. Such comments may include reports of errors in the PVT, suggestions for additional test cases, interpretation questions, or any other ideas on how to improve the PVT. Please send all correspondence, including questions about PHIGS validation and obtaining the PVT, to:

Project Leader, PHIGS Validation Tests

Computer Systems Laboratory

National Institute of Standards and Technology

Bldg. 225, Room A-266

Gaithersburg, MD 20899

This report identifies certain commercial software products in order to illustrate some of the concepts discussed herein. Such identification does not imply recommendation or endorsement by NIST. 


\section{STRUCTURE AND FORMAT OF PVT SUITE}

The PVT suite is implemented as a set of documentation files and source code files. This section describes the relationship among these files, and the format conventions they follow.

\subsection{Tree structure}

The PVT suite is organized in a hierarchical, or tree, structure. This tree closely resembles the organization of section 4 of the generic ISO PHIGS standard, which contains the basic conceptual description of PHIGS. See Appendix B for the complete description of the PVT tree.

Concretely, the tree structure is expressed by storing the modules of the PVT system in a hierarchical file directory, such as that supported by VAX/VMS, Unix, and MS-DOS. For illustrative purposes we shall adopt a neutral naming convention, wherein the levels of a file structure are separated by the " " character, for example: PVT 05 03 DOC.TXT as the name of the documentation file for module 05 03. As distributed, the root of the PVT tree contains a number of system files:

a. USER GUIDE.PRT: User's Guide for the PVT; the file you are now reading

b. SYS DICT.PRT: Dictionary file to specify the numbering of PHIḠS functions and data structures

c. PGM CHAR.PRT: Contains a list of all programs, explicitly numbered, together with their characteristics

d. INITPH.FOR: Source code for initializer of PVT configuration file

e. OPRCMT.FOR: Source code for program to allow entry of operator comments outside the usual context of a test program (see section 4.4 )

f. SUBLIB.FOR: Source code for global subroutine library

9. TRANS_SUBLIB.FOR: Source code for transformation subroūtine library

\subsection{Modules}

There is at most one PVT module per node in the tree. Every leaf node contains a module. A non-leaf node may contain a module, or may be empty, serving simply to organize the system. Every 
module contains exactly one documentation file, conventionally named DOC.TXT, and one or more program files, named P01.FOR, P02.FOR, etc. The documentation file contains the set of semantic requirements (SRS) for the module, together with the design for each of the module's programs. The programs contain the test cases (TCs) for the module. Format details are explained below. See Figure 1 for a schematic diagram of the structure of a module.

\author{
Module \\ DOC.TXT (Documentation file) \\ Semantic Requirements \\ SR1 \\ SR2 \\ ... \\ P01 design \\ P02 design \\ $\cdots$ \\ P01.FOR (Program file) \\ TC \#1 \\ TC \#2 \\ … \\ P02.FOR (Program file) \\ ...
}

Figure 1: File Structure of a Module

\title{
2.3 Subroutine Libraries
}

The only other entities of the PVT system not in the root, besides the documentation and source code files, are the subroutine libraries (also in source code) used by the main programs. These libraries are in the tree structure, and are associated not with individual modules, but with the set of modules below them in the tree or at the same level. Thus, if programs in modules 05 01 01, 05 01 03, 05 02, and 05 04 all use a library of common subroutines, this library will reside in PVT 05. Libraries are named SUBLIB.FOR. There is a global subroutine library, at the PVT level, containing routines used throughout the PVT system. Finally, there is a special-purpose library, anomalously named TRANS_SUBLIB.FOR in the root node, containing routines that simulāte the geometric transformation utility functions of PHIGS and perform other mathematical functions.

The subroutines are self-documented; each starts with a brief description of its purpose and of the meaning of its parameters. See Appendix $D$ for a dictionary of the names and locations of all PVT subroutines. 


\subsection{Order}

There is never any order-dependence between modules, nor is there a required order for program execution within a module. Following the PVT tree structure, depth-first, may be the most natural way to proceed through the modules, but there is no requirement to do so.

\subsection{Module Documentation}

Module documentation is written for a reasonably knowledgeable PHIGS user. Questions about PHIGS itself must be answered by reference to the standard [PHIGS89].

Figure 2 contains a schematic outline of the module documentation. Refer to it, or to an actual example of module documentation when reading this section.

\subsubsection{Introductory Headings -}

The first heading, "TITLE", contains a brief phrase describing the main topic of the module. The second heading, "MODULE\#" contains the identifying number of the module, which may be simply related to its location in the file hierarchy, e.g., the files of MODULE\# 02.01 .02 are in the PVT 02 01 02 sub-directory. All level numbers are two-digit. The third heading, "DESCRIPTION" contains a free-format paragraph explaining the scope of the module.

\subsubsection{Semantic Requirements -}

Next comes the list of semantic requirements associated with the PHIGS feature under test. These are assigned sequential identifying numbers, which will be referred to by the relevant TCs. Each SR is a simple declarative sentence, stating some requirement on the behavior of conforming implementations. Although intended to be clear and unambiguous, these have to be read in a "reasonable" way, with appropriate assumptions about context. The SRs are not "conditionalized" into absolute truths by explicitly stating all the normal pre-conditions that may apply (e.g., if a structure is open and if the system has space left to allocate, and if there is no power failure, then ...).

The SRs use the terminology of the standard when referring to functions or data structures. By convention, angle brackets are used when referring to the generic names of functions, e.g., <set element pointers. Note that the words "valid" and "realizable" have a technical meaning: the former indicates simply that a parameter is accepted by a function without generating an error, 


\section{TITLE:}

MODULE\# :

DESCRIPTION :

SEMANTIC REQUIREMENTS:

SR1:

\#F functions

\#D data structures

\#S references to standard

\#T test cases

$\# \mathrm{X}$ module cross-references

\#C comments

SR2 :

\#F

\#D

\#S

LOCAL DICTIONARY:

SEMANTIC CROSS-REFERENCES: (if applicable)

LOCAL SUBROUTINES: (if applicable)

PROGRAM 1

CHARACTERISTICS :

OPERATOR SCRIPT:

DESIGN :

TEST: \#SR

"Text of test case message."

...

TEST: \#SR

END PROGRAM I

PROGRAM 2

...

END PROGRAM 2

Figure 2: Format of Module Documentation

while the latter indicates that the implementation must actually be able to render the graphical attribute in question. The wording of the SR applies to the generic standard, and is not language-dependent.

Immediately following each SR there are a number of associated fields, one per line, each prefaced by a "\#". The first four of these, \#F, \#D, \#S, and \#T are mandatory. 


\subsubsection{Related Functions -}

\#F is used to list the functions associated with this SR, i.e., those whose behavior is at least partially constrained by the SR. Functions are referred to by an identifying number (1-321) which corresponds to the order in which they appear in section 5 of the standard. The SYS_DICT.PRT file contains a complete table of function numbers.

\subsubsection{Related Data Structures -}

The \#D entry lists the data structures which are relevant to this SR, i.e., those whose contents are changed or inspected in the course of checking the SR. The data structures are referred to by an identifying hierarchical number which reflects the organization of section 6 of the standard. The SYS DICT.PRT file contains a complete table of data structure numbers. Note that the data structure number need not be a leaf node. If the SR affects everything under a non-leaf node, then an entry for that node implicitly covers all those below it.

\subsubsection{Basis In The Standard -}

The \#S entry lists those parts of the standard upon which the $\mathrm{SR}$ is logically based. These references are in the form: <section-number> / <page-number> / <paragraph-number>, all of which refer to the 1989 ANSI/ISO PHIGS standard, [PHIGS89]. The rules for counting paragraphs within a page of the standard are as follows. Whatever partial chunk of text is at the top of the page is number 1. Subsequent paragraphs are deemed to start by a blank line (even if half-height) followed by text at extreme left hand margin (not indented), but not counting section titles. Thus, page 38 has 7 paragraphs: 1 partial completing section 4.5 .1 , and 2-7 in section 4.5.2. Figures are associated with the preceding paragraph. For example, page 46 has 3 paragraphs with figure 7 as part of the first.

The page numbers of the relevant functions, as listed under \#F, are not included; if a function is relevant, it is to be assumed that its description in section 5 of the standard will have some bearing on the SR. If there is no basis in the standard for the SR besides the description of the relevant functions, an "n" appears in the \#S entry. 


\subsubsection{Test Cases -}

Each SR, under the \#T entry, lists the TCs which depend on it. The format of each TC reference is: $\mathrm{P}$ <program-number> / <test-case-number>. The test case number simply refers to its sequential position in the text of the program. Note that not all TCs are always executed; under certain conditions specific to the implementation, some may be skipped.

\subsubsection{Module Cross-references -}

In some cases an SR may be significantly related to the topics of several modules. When this happens, we assign the SR to the most strongly related module, and use the \#X entry to list the other relevant modules.

\section{5 .2 .6 Comments -}

The \#C entry is for free-form comments to allow explanation of any unusual aspect of the SR. For instance, if the support in the standard for the SR is obscure or indirect, the comment field might be used to explain the validity of the SR.

\subsubsection{Local Dictionary -}

Since the \#F and \#D entries under the sRs are not self-explanatory, the documentation supplies the subset of the global function and data structure dictionary needed to decode the entries of this module.

\subsubsection{Semantic Cross-references -}

If this module is pointed to by an entry in the \#x field of another module, that pointer is noted here in the format <module-number>/SR<sr-numbers. For example, if SR4 in module 02.01 contains a \#X entry for module 04.01.01.01, then the latter module will have "02.01/SR4" as a semantic cross-reference entry.

\subsubsection{Local Subroutines -}

If the programs of this module use any local subroutines (i.e. any besides those in the root-level global library), it is noted here. For detailed documentation of the logic and parameters of subroutines, please see the source code. 


\subsubsection{Program Design -}

The second major part of the documentation of the module is a description of the programs and TCs which actually test the SRs of the module. The programs are numbered sequentially. The design is intended to be language-independent, relying only on the generic standard and not on language binding details.

\subsubsection{Headings -}

Each program design starts with a "PROGRAM" header, which includes the ordinal number of the program within this module and a descriptive title. The next entry, "CHARACTERISTICS" contains a four-character code (" $y$ " or " $n$ " for yes or no) to indicate various properties of the program:

1. requires graphical output features of the primary workstation, which must be of category OUTIN or OUTPUT,

2. requires graphical input features of the primary workstation, which must be of category OUTIN or INPUT,

3. the presence of incidental non-graphical input, i.e., the need to gather information at run-time from the operator, but where the input facilities themselves are not under test

4. the need for visual (or other human) interpretation, i.e., the program cannot completely determine pass/fail results, but must rely to some degree on the operator

The CHARACTERISTICS are useful in setting up procedures to run the test suite. Programs with "--nn" are passive tests and may be run without operator intervention. Programs with "nn--" are workstation independent tests and need not be repeated when testing various workstation types (see section 4.1).

The last heading is "OPERATOR SCRIPT." This contains the instructions to be followed by the operator when running the test. If there is no need for operator intervention, then this entry will say "passive test." See section 4.4 .3 for general operator instructions.

Each program design is terminated explicitly by an "END PROGRAM" heading, followed by the identifying ordinal number of the program. 


\subsubsection{Logic And TCs -}

The core of each program design is a body of pseudo-code which describes the flow of logic and data representation within the program. It should be clear from this pseudo-code why the embedded TCs are supposed to work. The pseudo-code describes only the logic of the program relevant to the TCs; incidental processing, such as opening PHIGS, or opening a structure is not included. The goal is to give the user an understanding of the basic logic of the code, not to depict all the programming details. For the latter, one can consult the code itself.

The style of the pseudo-code is meant to be informal and self-explanatory. Only a few common control structures are used, such as looping, if-then-else, and goto. Labels (the object of goto statements) begin in column 1 and terminate with a colon. The heading "TEST:" heralds the beginning of a test case. This is followed on the same line by "\#SR" and then a list of the SRs upon which this TC is logically based. Beginning on the next line is the textual statement of the expected (correct) result of the test, surrounded by double-quotes. The text is unique within the program and thus serves as the identifier of the TC.

For interactive tests, prompts to the operator are indicated by "OPQA" (for operator question and answer), followed by a slash, a topical heading in upper case, and then the question itself, e.g:

OPQA/EDGE FLAG INDICATOR: Which triangles have visible edges?

The result of each test is recorded by executing either a "pass" or "fail" procedure, denoted in the pseudo-code simply by those words. Every TC should cause execution of either one or the other (but not both, of course) of these. In many cases, where the result depends directly on a single condition, instead of coding:

$$
\begin{aligned}
& \text { if (condition) then } \\
& \text { pass } \\
& \text { else } \\
& \text { fail } \\
& \text { endif }
\end{aligned}
$$

we use the short-hand form:

pass/fail depending on (condition)

\subsection{Source Code Conventions}

In this section we discuss characteristics which pertain to the source code throughout the PVT system. The code itself is written to be comprehensible by a PHIGS- and Fortran-literate reader. 


\subsubsection{Language -}

The language of version 2 of the PVT system is full ANSI Fortran (ANSI X3.9-1978) as defined in [FORT78]. The language binding to PHIGS is the full Fortran binding [PHFOR90].

\subsubsection{All Variables Declared -}

Although not required by the Fortran standard, the code explicitly declares the type of all program variables. We believe this is useful for several purposes: it helps avoid certain programming errors (e.g. misspelled variables can be detected by having the compiler flag undeclared variables), it enhances self-documentation of the code, and it may prove useful for automatic conversion to other languages, such as C.

\subsubsection{Standard PHIGS Names -}

Where appropriate, the code uses the standard symbolic constant names recommended in section 6 (Enumeration Types) of the Fortran binding standard [PHFOR90].

\subsubsection{Dummy Parameters -}

When the code invokes a PHIGS function some of whose output parameters are not used in the subsequent logic, these parameters are assigned names in the format: <type>DUM<digit>, where <type> is "I" for integer, "R" for real, "L" for logical, and "C" for character, and where <digit> is some differentiating digit. This tells the reader which parameters are relevant to the logic of the program and which are incidental.

\subsubsection{Program Banner -}

Each test program starts with comment lines forming a banner. This banner identifies the program with a unique "TEST NUMBER" and a "TEST TITLE". These appear in a box of asterisks. The format of the test number is <module-numbers / <program-number>. For example, "04.04.01.02/03" identifies program number 3 in module 04.04.01.02. Thus, the program's file name is P03.FOR in directory $\mathrm{PVT} \sim 04 \sim 04 \sim 01 \sim 02$. 


\section{6 .6 Common -}

Each test program contains a set of identical declarations for certain variables in the common areas GLOBNU and GLOBCH. These are used to convey information within the system. For a full description of each of these global variables, see Appendix A.

\subsection{Ubiquitous Subroutines}

In this section, we discuss briefly the function of some of the more commonly-used subroutines. Since these are used throughout the PVT system, anyone wishing to understand the code should be familiar with their purpose. There are many other subroutines, however. All PVT subroutine libraries are self-documented, so when questions arise, the code itself should be consulted.

\subsubsection{Initialization And Clean-up of Test Programs -}

Normally, the first executable statement within a program will be a call to INITGL. This routine performs all the work needed to set up the environment for the program; in particular, it initializes the values in the common areas GLOBNU and GLOBCH so that they may be used freely (e.g., workstation type needed to open a workstation). It does this by reading certain constant values from the PVT configuration file (see section 4.2 on Running INITPH). The program supplies its identifying TEST NUMBER (see section above on Banner) as the single parameter.

For interactive programs, the SETDLG subroutine sets up the dialogue area as requested in the configuration file, and initializes the DIALOG common area.

The last executable statement is a call to ENDIT. This closes all open workstations, closes the currently open structure, and closes PHIGS. It then calls WINDUP to write out summary results, close files, and perform any other processing needed to finish the test program.

\section{7 .2 TC Subroutines -}

There are a number of subroutines needed to implement a given TC in the code. These correspond closely to the way a TC is set up in the pseudo-code. The SETMSG subroutine sets up a "current TC message" for the condition about to be tested, which contains both the SR references and the text describing the condition under test. If a test case is currently being checked for adherence to the standard, the test message will include "[UNDER REVIEW]". 
Depending on the results of the TC, the program then executes either the PASS or FAIL subroutine, which records the result. The IFPF subroutine is a shorthand form, which accepts a single logical expression as a parameter and invokes PASS if it evaluates as true, and FAIL if false.

\subsubsection{Message Subroutines -}

The only output of each test program is a series of messages. The following subroutines generate messages of the indicated type:

\begin{tabular}{|c|c|c|}
\hline Subroutine & Message-type & Function \\
\hline INITGL & SY & initialize program \\
\hline ENDIT & SY & finalize program \\
\hline PASS & OK & reçord TC passed \\
\hline FAIL & FA & record TC failed \\
\hline IFPF & OK or FA & record TC result \\
\hline INMSG & IN & information \\
\hline UNMSG & UN & abort \\
\hline NCMSG & $\mathrm{NC}$ & abort \\
\hline CHKINQ & NC & continue or abort \\
\hline
\end{tabular}

See section 4.4 .4 on for the interpretation of messages and message-types. The subroutines UNMSG and NCMSG are used when the program must be aborted, as opposed to normal program conclusion which is done via ENDIT. No further code is executed after either of these is encountered in the flow of control.

\subsubsection{CHKINQ -}

It is very common within the PVT system for an inquire function to be incidental to the main purpose of the test. Since the test relies on the result of the inquire, we wish to ensure that it has completed successfully. Every incidental use of an inquire, therefore, is followed by CHKINQ, which simply checks that the error indicator from the function is zero. If not, CHKINQ invokes NCMSG and therefore aborts the program. Otherwise there is no effect.

of course, when an inquire function is being purposefully tested, its error indicator is checked explicitly as part of the usual PASS/FAIL determination. 
This section covers the steps needed to install the PVT code on a typical computer system. The advice is necessarily general, since many aspects of installation are system-dependent. Installation consists of the following steps:

1. Copy files into hierarchical directory

2. Customize code, especially naming of configuration file

3. Set up procedures for automatic execution of source code

\subsection{File Hierarchy}

Copy the PVT files from the medium on which they are delivered to the hosting system. If the medium is magnetic tape, there is an accompanying information sheet to describe the physical format of the files.

Store the files in a hierarchical directory as described in section 2.1 and Appendix B. In particular, use the same two-digit level numbers as in the files' own self-identification. The name of the PVT root level is arbitrary; a name like USER PVT ... is a reasonable choice. See section 2.1 for a list of the files that belong in the PVT root directory. The global and module-level documentation need not be stored on-line, although this may be convenient for automatic searching for entities within the PVT suite.

Assign the source code filenames in the format "Pxx.FOR" where "xx" indicates the ordinal number within the module, and ".FOR" indicates the source language. For example, the program with the header

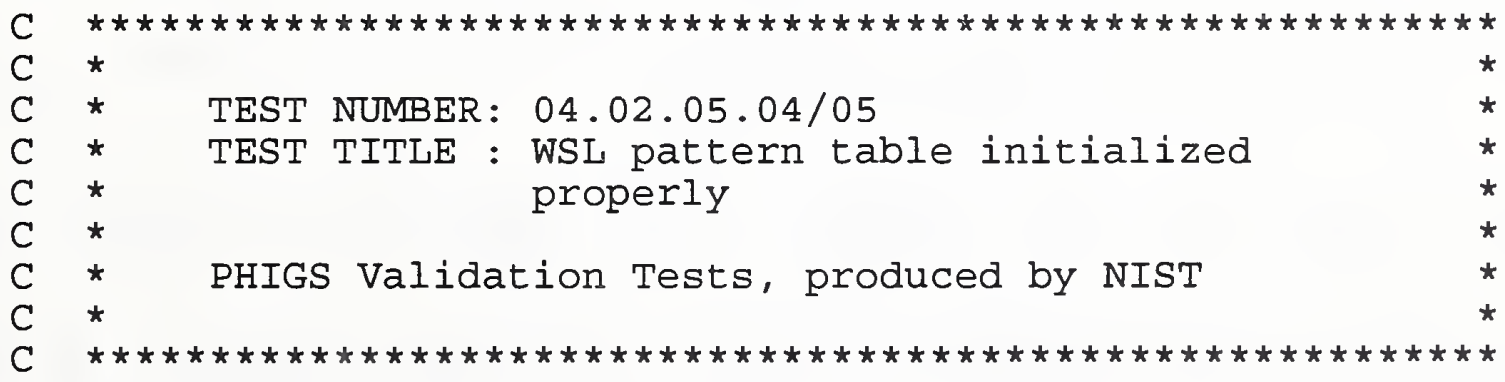

should be stored as "USER PVT 04 02 05 04 P05.FOR". Different operating systems have various conventions for the suffix to denote Fortran source code, such as ".f" or ".FTN". Any such convention is acceptable as long as it is consistently applied. It is strongly recommended, however, that the initial part of the name be in the format "Pxx". Throughout the PVT documentation, programs 
are always identified according to this format, and it avoids confusion if the program's physical name matches its logical identification.

Likewise, copy subroutine libraries into the appropriate tree nodes. The banner of each subroutine identifies the correct node for the library. The recommended file name is SUBLIB.FOR.

When done, check your file hierarchy against the PVT tree structure as described in appendix $B$ to ensure that all the files have been copied.

\subsection{Customization of Code}

Some of the source code may need to be changed in order to run on your system. These places are noted in the code by a comment line beginning with "CMOD". Use any text editor to perform these changes. The first change, naming the PVT configuration file, is mandatory for all systems. All the other changes are optional.

\subsubsection{Naming PVT Configuration File -}

First, choose a name for the PVT configuration file. The name you pick must be absolute, i.e., it must be valid when used from any part of the hierarchy. We recommend locating the PVT configuration file in the PVT root.

This name must be inserted in three locations all of which are in the PVT root:

1. the INITPH.FOR program which writes the file

2. the INITGL subroutine which reads the file and is located in the global SUBLIB.FOR file

3. the MULTWS subroutine which reads the file, also located in the global SUBLIB. FOR file

In all three cases, search for the string "INITPH\$DAT" (the name we used) in these routines to locate the insertion point.

The PVT configuration report file is a human-readable version of the PVT configuration file. Pick a name for it as well, such as "INITPH.PRT", and insert it into the INITPH.FOR program. 


\subsubsection{Special Processing For Opening PHIGS -}

All the test cases call XPOPPH to open PHIGS, rather than the standard POPPH. The XPOPPH subroutine, as delivered, simply calls POPPH. If your system has special processing requirements for accessing PHIGS, these may be addressed by additions to XPOPPH. Note, however, that for validation purposes, such changes are subject to approval by NIST.

\subsubsection{Random Number Generator -}

The last routine in the global subroutine library, RND01, is written in non-standard Fortran, since the standard provides no way to randomly initialize a seed for a random number generator. You may alter RNDO1 (possibly using a local non-standard time function) so that the seed is different for each execution. This is the preferred mode of execution. All the other random-number routines are built on RND01, so if it works, the others need not and should not be changed.

\subsubsection{Naming Individual Message File -}

If you request that the test programs generate individual message files (see section 4.2.3) INITGL will, by default, form the name of the file by using "P" as a prefix, the two-digit ordinal number of the program, and ".MSG" as a suffix; e.g., P04.FOR will write to P04.MSG. If you prefer another naming convention, search for ".MSG" in the INITGL routine and change the code accordingly.

\subsubsection{Resolution of Parameters For <Open Workstation> -}

The INITGL and MULTWS subroutines read the PVT configuration file in order to determine the parameter values needed to open the primary and secondary workstations (workstation identifier, connection identifier, and workstation type), and report these back to the calling program. INITGL sets the value of three variables in COMMON to do this, while MULTWS returns the values in its output parameters. In both cases, the assumption is that the correct values are static and can be set once by the INITPH procedure (see section 4.2 , below). If your system is such that this information can be determined only at run-time, you must re-code the relevant sections of INITGL and MULTWS, so that they still deliver the required values. 


\subsubsection{Providing Valid Names For Archive Files -}

The subroutine AVARNM, in node 03 of the PVT tree, must return to the caller an integer representing the valid name of an available empty archive file. The code assumes that this name should be interpreted as a Fortran logical unit number. If your system has a different interpretation, or has special requirements for opening an archive file, you must modify this subroutine accordingly.

\subsubsection{Time-stamping Message Files -}

The Fortran standard provides no function for determining time or date. If, however, your implementation does provide such a feature and you wish to include this information in the PVT output, we suggest you alter the INITGL subroutine at the point where it formulates the header system message. This is done at the very end of the subroutine, in the last call to BRDMSG.

\subsubsection{Operator Communication -}

The OPMSG and OPYN routines in the global SUBLIB.FOR library write messages to and read messages from the operator. Because a workstation may not be open at the time these are executed, the PVT code resorts to the use of Fortran's print and read statements. If there is a better way to send a character string to and from the operator in your system, you may re-code these routines accordingly. If print and read work well within your system no change is necessary.

\subsubsection{End of File -}

If you specify a global message file (see section 4.2.3), the INITGL routine in SUBLIB.FOR must position the file pointer at end of file so as to append new messages. In standard Fortran, the only way to do this is to read through the whole file. If your Fortran system provides a more efficient (though non-portable) way to do this, you may wish to substitute that method in INITGL. Otherwise, no change is needed.

\subsection{Linking Subroutines}

In most systems, you will wish to compile all the subroutine files (TRANS_SUBLIB.FOR and SUBLIB.FOR in the root and all the local SUBLIB.F.F files) so as to create subroutine libraries, which may then be linked in with each test program. Of course not all 
the test programs use all the subroutines, so do whatever your system requires such that only needed subroutines are linked in.

We strongly recommend setting up a command procedure to compile, link, and execute a test program solely by referring to the name of the program. In particular, all local subroutine libraries (those above the program in the directory tree) and the global libraries must be made available, as well as linking to the code of the PHIGS implementation. Note that the global SUBLIB.FOR contains a PERHND subroutine - the standard PHIGS-Fortran name for an error-handler. Some PHIGS implementations may require special treatment to link in PERHND. Since most linkers prefer to be given access to libraries in order from caller to called procedure, you should arrange access first to the local PVT libraries, then the global PVT libraries, and finally to the PHIGS library. See Appendix E for two examples of how this might be done.

The local subroutine libraries are assigned to nodes of the tree only for the purpose of clarifying the logical relationship among the test programs and subroutines. If linking in several libraries is difficult in your system, you can simply concatenate all the subroutine source code together as one large file, and compile it as one library, presumably in the root. No two PVT subroutines have the same name, so no name clashes will occur.

\section{OPERATION}

In this section, we cover the steps needed to run and interpret the PVT system. Like other conformance test systems, the PVT is not, nor can it be, a totally automated process. The PVT code and documentation are best seen as components of an integrated and interdependent system, which includes the operator as its active, directing component. We include in the notion of operation the process of interpreting the behavior of the programs, particularly with regard to conformance.

\subsection{PVT Sessions And Workstations}

Let us refer to the execution of a set of PVT programs as a session. For each session, a primary workstation must be specified, together with an optional set of secondary workstations. The primary workstation is the one whose features are thoroughly tested by the PVT programs. Secondary workstations are tested only by certain special-purpose programs such as those dealing with multiple workstations. Thus, for each workstation to be tested in depth, the operator must run a distinct session.

In order to conform, a PHIGS implementation must support at least one workstation of category OUTIN. No other workstations need be supported. If other workstations are accessible, however, 
they must have the capabilities associated with their categories.

Thus, a typical scenario for conformance testing is first, a session in which an OUTIN workstation is designated as primary and in which all the PVT programs are executed. Such a session checks the minimum requirements for conformance.

Subsequent sessions deal with the capabilities of other workstations of whatever category. Each of these workstations is designated in turn as primary and an appropriate subset of the PVT is executed for it. Since there is no need to re-run tests which are not relevant to the capabilities of these other workstations, not all PVT programs need be executed in these subsequent sessions. Use the information in the CHARACTERISTICS entry of the program design documentation (summarized in appendix $C$ and in the PGM_CHAR.PRT file) to decide which programs are pertinent, according to the table below.

\begin{tabular}{ll} 
Workstation & \\
Category & CHARACTERISTICS \\
\hline OUTIN & yn-- or ny-- or yY-- \\
OUTPUT & yn-- \\
INPUT & ny--
\end{tabular}

Programs with a CHARACTERISTIC entry of "--nn" require no operator interaction and may be run in batch mode; otherwise there must be an operator for the test. By convention, within a module, all the passive programs precede the interactive ones (e.g. P01-P05 are passive, P06-P09 are interactive).

It may prove useful to construct a batch procedure for the passive tests of each category of workstation, so that you can automatically invoke the correct subset of programs.

\subsection{Running INITPH For PVT Configuration}

The PVT Configuration file contains the information which is specific to the PHIGS implementation being tested, but the same for all test programs within the PVT session. Its purpose is to allow an operator to specify such information only once at the beginning of each session, rather than repeating it for each program. The INITPH program creates this file, based on the operator's responses. The program is stored as INITPH.FOR in the PVT root. It uses some subroutines from the global subroutine library, and also from PHIGS itself, so these libraries must be available as it is compiled and linked. Execute INITPH, and respond as prompted. Most responses are in the form of an integer. You should be prepared to supply the following information to INITPH: 
1. parameters for <open phigs> (error file and memory units)

2. number of workstations accessible in this session

3. <open workstation> parameters for each accessible workstation (workstation identifier, connection identifier, workstation type)

4. whether to suppress "pass" messages

5. choice of destination(s) for messages (screen, individual files, or global file)

6. logical unit numbers for individual message files (if used)

7. file name for global message file (if used)

8. maximum line length for messages

9. whether to control the tests with a pseudo-random or true random number sequence

10. whether the primary workstation is capable of visual output

11. method for prompting the operator, and maximum line length for prompts

12. method for operator responses to prompt

13. location and size of the dialogue and echo areas

14. ratio of meters to DC units for the primary workstation

\subsubsection{Parameters For Opening PHIGS -}

The first two questions concern the input parameters to be passed to the <open phigs> function, whenever that function is needed in a PVT program. Simply supply the values your implementation requires.

\subsubsection{Parameters For Opening Workstations -}

The next questions concern the workstations accessible to this implementation. Tell INITPH the total number of accessible workstations (primary and secondary). Then, for each of these, supply the three parameters by which <open workstation> can open it: workstation identifier, connection identifier, and workstation 
type. Be sure that the first set of parameters refers to the primary workstation.

\subsubsection{Control of Messages -}

Next, INITPH will ask you whether you want a message to be generated whenever the implementation successfully passes a TC in a test program. You can specify either that such messages are always suppressed, always generated, or that each program asks the operator what to do, so that he or she can selectively suppress pass-messages at run-time. No other type of message can be suppressed.

The next questions deal with the destination of messages as they are generated. First, indicate whether or not messages are to be sent to the operator (typically on the screen). Next, indicate to which files messages should be written. Individual message files are created once per execution of a test program. By default they are given the same name as the program, but with a "MSG" suffix, rather than "FOR". The global message file is a cumulative file to which messages are appended whenever a test program is run.

These are independent choices; messages can be sent to any combination of the three destinations: operator, individual file, or global file. Each enabled destination receives exactly the same set of messages.

If you specify a global file, you must then provide an absolute name for this file, so that all programs can write to it. You may want to specify a distinct name for the global message file of each PVT session. This response is, of course, not in the form of an integer, as are the others. Since some operating systems have reserved logical unit numbers in Fortran, you are also asked to provide these for the individual and/or global file, if they have been designated as destinations.

You must specify the maximum number of characters per line which should be generated when the PVT system formats a message. Some messages may be quite long and would not fit on a reasonably-sized single line. Message text is never truncated; rather, it is simply broken into lines of the specified size.

\subsubsection{Control of Randomization -}

Many of the interactive tests randomize the choices presented to the operator so that the correct responses are not predictable (see section below on operator interaction). For some purposes, however, it is desirable that the tests execute with repeatable displeys and operator prompts. If you want to get repeatable behavior, enter a real number between 0.1 and 0.9 . This value will 
be used as the seed for a random number sequence. Thus, re-initializing to a distinct value between 0.1 and 0.9 will cause repeatable behavior within the new session, but distinct from that of the previous session.

Entering any value outside the range of $0.1-0.9$ causes the system to use a time function to set the seed for the random sequence, and thus generates truly random operator choices.

\subsubsection{Primary Workstation Support For Graphical Output -}

If the primary workstation is capable of visual output, answer yes, otherwise no. The normal answer is "yes". The question is here to allow for testing of INPUT-only workstations and metafile workstations in later versions of the PVT.

\subsubsection{Control For Prompting The Operator -}

When running the interactive tests, the system poses questions to the operator. This option lets you choose the mechanism for transmitting those questions: 1-Fortran print, 2-PHIGS <message>, or 3-PHIGS <text>. In many systems, Fortran PRINT will write to another window. PHIGS <text> will use some of the PHIGS display space for questions, possibly leaving less room for the picture under examination.

If using Fortran print or PHIGS <message>, you must specify the maximum number of characters per line in interactive prompts.

\subsubsection{Control For Operator Responses To Prompt -}

You may specify the means by which the operator is to respond to prompts: either Fortran-read or PHIGS <request string (and the device number for <request string>).

\subsubsection{Location And Size of The Dialogue And Echo Areas -}

If you are using PHIGS for operator input or output, you must specify where the dialogue area (containing operator prompts and responses) should be. The choices are: 1-dialogue at bottom of screen, 2-dialogue at right, or 3-toggle picture and dialogue so that the screen can alternate betweem the two. Since the picture area will use the largest square remaining on the screen after the dialogue area has been reserved, it is recommended that the dialogue area be on the right for wide screens, and on the bottom for tall screens. 
You must then specify what percentage of the screen should be reserved for the dialogue area. Something in the range of 15-30 percent is usually a reasonable choice.

Finally, if you are using PHIGS for operator input, specify what percentage of the dialogue area is to be reserved for the echo of operator responses (the remaining area is used for prompts). Since prompts are usually larger than the responses, something like 10-20 percent is a good choice.

\title{
4.2.9 Ratio of Meters To DC Units For The Primary Workstation -
}

You must tell the system the ratio of meters to DC units for this workstation. You may either enter the number directly, or physically measure a diagonal line on the screen, and INITPH will compute the result.

At the successful conclusion of INITPH, the operator receives a report on the names of the files to which the PVT configuration file and PVT configuration report file (the human-readable version) have been written.

\subsection{Background Documentation}

\begin{abstract}
In order to understand the significance of the programs' output for conformance, you should read over the module documentation and those sections of the standard referred to in the \#S entries of the SRs. You will then have the appropriate background information to allow you to focus on the details of each test result.
\end{abstract}

\subsection{Execution And Interpretation}

Invoke the system-dependent command(s) needed to compile, link and execute each program in the session. There are a number of possible outcomes, each of which are dealt with below. We assume throughout the following sections that the PVT programs are themselves valid. If you encounter some aspect of a program which you believe is incorrect, please submit a comment to that effect as described in section 1 .

\subsubsection{Failure To Compile Or Link -}

If your compiler does not support some feature of ANSI Fortran [FORT78] used by a PVT program, you must find a different way to perform the equivalent operation. Note, however, that such lack of 
support constitutes non-conformance, since the Fortran binding [PHFOR90] incorporates the Fortran standard [FORT78]. Similarly, problems in the system software which performs linking of subroutines must be resolved by you. Any problem with system software which prevents a valid invocation of a PHIGS function by a program renders the implementation non-conforming.

Uncorrected failure to begin execution of a program should be noted by running the OPRCMT program. This will record operator comments as messages in the specified destination files. Therefore the results of all tests (even non-executing tests) are saved in a uniform way. Even though the OPRCMT program resides in the PVT root, you should be in the sub-directory of the appropriate module when executing OPRCMT so that the individual message file will be written in the correct location. For example, suppose program 06.01.02/01 cannot be executed. Then, while still in that sub-directory, execute OPRCMT, enter the identifier of the module and program ("06.01.02/01"), and then enter your comment(s) as prompted by the program.

\subsubsection{Failure To Complete Execution -}

The PVT programs are written conservatively so as to fall well within the operational limits of any reasonable PHIGS implementation. Furthermore, the PERHND subroutine should handle any errors signalled by PHIGS functions. Therefore, it is to be expected that every PVT program, once started, will proceed until completion under the control of the appropriate PVT subroutine, such as ENDIT for successful completion, or UNMSG for aborted completion.

If the PHIGS system itself aborts execution, it means that the implementation fails the test and does not conform, since no unhandled PHIGS error conditions should arise within the PVT code. If not otherwise evident, this outcome is indicated by the absence of two SY-type messages (the first containing the error count, the second to identify PVT completion) at the end of the message file $(s)$.

You may wish to use the OPRCMT program (see preceding section) to enter an operator comment on the cause of failure, but be aware that the individual message file generated by the original program may be over-written. The cumulative message file will contain the output of both the aborted program and OPRCMT.

\subsubsection{Operator Interaction -}

The interactive tests all present a graphical display to the operator in the picture area of the screen and then ask him/her a question about it. See the OPERATOR SCRIPT entries in the DOC.TXT 
files for detailed instructions if the screen is not self-explanatory.

\subsubsection{Format For Answering Questions -}

In general, the format of the response should be obvious from the question. Most questions are single integer choices, such as "Which fill area is different?" referring to a display of perhaps 8 numbered fill areas. Answer such questions with a single integer, in the example given, 1 through 8. If there is no unique correct answer (e.g. all fill areas are the same, or several differ from each other, or the screen is blank) respond with "0" (see below).

When a list is called for (e.g. "Which primitives are highlighted?"), enter a list of integers separated by commas, e.g. $" 2,3,5,6 "$. If the order of the list is significant, the prompt will so indicate. The single character "n" denotes the empty list (e.g. your response should be "n" if none of the displayed primitives were highlighted).

Other response types are: real numbers, "y" or "n" for yes/no questions, and character strings. If a response has an incorrect format, PVT issues a warning and lets you re-enter the response.

\subsubsection{Special Processing of Operator Responses -}

Except for yes/no questions, which require " $y$ " or "n", a response of "O" is always valid and means that the question is unanswerable. After a "0" response, the operator is given an opportunity to enter a free-form comment explaining the nature of the problem. The comment is then recorded in the output message file. An operator comment is entered as several text lines, and terminated by a line containing a single period. If the first line of the comment is the termination line, no message is generated. Usually a "0" response causes the associated test case to fail.

Also, the operator may respond to any prompt with "@" (at-sign). This allows him/her to enter a comment pertaining to the current picture and prompt, but without causing failure as would the "o" response. The comment is made before actually answering the question. As with "0", the comment is recorded as a message and terminated by a line with a single period. After entering the comment, the original question is put to the operator again and must still be answered.

When the operator has indicated to INITPH that the dialogue and picture area are to be toggled (see section 4.2.7), the toggling is controlled by entering "t" or "T". The effect is to switch between displaying the dialogue area and the picture area. The actual answer may be entered when either area is visible. 


\subsubsection{Completion of Execution And Message Type -}

The PVT programs sometimes examine whether they can proceed to execute, or whether anomalous conditions have arisen such that further execution is futile. In the latter case, before stopping, the program will issue a message of type UN or NC, indicating the reason for aborting further testing. Normally, all the TCs within the program that are appropriate for the implementation are executed.

In either case, the output of a completed PVT program consists of a sequence of messages. These messages are sent to the destinations specified in the PVT configuration file. They are followed by three records each consisting of a single period. This serves to separate visually the output of each test in the global message file.

The messages are categorized into six types in order to help the operator assess their significance. The output is formatted so that these types appear in the leftmost columns, with the text of the messages indented. The following table describes the circumstances associated with each of the message types.

\section{Message Types}

SY: System message, generated by INITGL and ENDIT. These messages herald the beginning and completion of execution of a PVT program. The next-to-last message contains a summary of the number of TCs executed and failures detected.

OK: The standard message resulting from a passed test case, generated by PASS or IFPF. INITPH allows the operator to suppress messages of this type.

FA: The standard message resulting from a failed test case, generated by FAIL or IFPF.

IN: Informational message, generated by INMSG or SIGTST.

Some situation was encountered which, while not erroneous, is worth noting, for example, the implementation is such that a certain condition cannot be tested. Also, this type of message can be used for the purpose of conveying useful but not conformance-related information about the implementation, such as support of optional PHIGS features.

OP: Message as entered by the operator, either as a result of entering "0", or "@" in response to a prompt. Typically, this message is used to record some unusual or unexpected visual feature of the display.

UN: Unanticipated error message, generated by UNMSG. This message is generated when the program detects some 
anomalous condition that prevents further processing. No inference is to be drawn concerning implementation conformance.

NC: Unanticipated non-conformance error message, generated by NCMSG or CHKINQ. The program must abort because a feature of PHIGS which is not under test, but needed for the test, has failed. Since the incidental feature is mandated by the standard, its failure indicates non-conformance. Most commonly, this message type is generated by CHKINQ when a required inquj.re function fails.

The operator may inspect the output at several levels of detail. In the simplest case, he or she may simply look at the error count at the end, to see whether or not all tests were passed. By scanning the left column for FA or NC type messages, the operator can focus on problems of non-conformance and ignore the other output. Finally, one can note the content of informational messages, which tests were passed or failed, which SRs are implicated in the result, and what the precise conditions of the test were.

\subsection{Customization For Debugging}

Users who are interested in debugging as well as conformance checking may wish to augment the tests so as to produce more detailed information. We recommend that the extra output from such enhancements follow the usual conventions of the PVT system. In particular, we recommend that the information be formatted into a character variable (using Fortran's WRITE statement) and then written out as an IN type message with the INMSG subroutine. There are many examples of this in the normal code. See these if you need further guidance. 
[CUGI90] John Cugini, Mary T. Gunn, Lynne S. Rosenthal, PHIGS Validation Tests (Version 1.0): Design Issues, NIST SP 500-181, National Institute of standards and Technology, Gaithersburg, MD, 1990.

[CUGI91] John Cugini, Interactive Conformance Testing for PHIGS, Eurographics' 91 (ed. F. H. Post and W. Barth), Elsevier Science, New York, NY, 1991.

[FORT78] Programming Language FORTRAN, ANSI X3.9-1978, American National Standards Institute, New York, NY, 1978.

[GKS85] Computer Graphics = Graphical Kernel System (GKS) Functional Description, ANSI ${ }^{-} \overline{X 3.124-1985,}$ American National Standards Institute, New York, NY, 1985.

[GKST89] GKS Validation Test Suite, Version 2.1, National Institute of standards and Technology, Gaithersburg, MD.

[PHFOR90] Information processing systems - Computer Graphics Programmer's Hierarchical Interactive Graphics system (PHIGS) language bindings = Part 1: FORTRAN, ISO/IEC 9593-1:1990, American National Standards Institute, New York, NY, 1990.

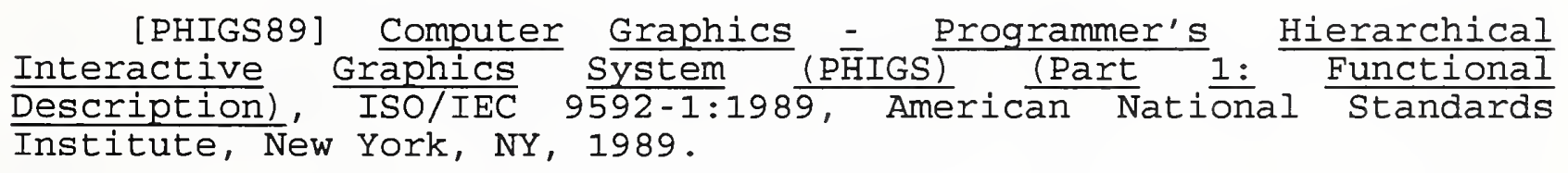


APPENDIX A

GLOBAL VARIABLES

Below is a description of the variables in COMMON in the Fortran source code. They are used globally throughout PVT.

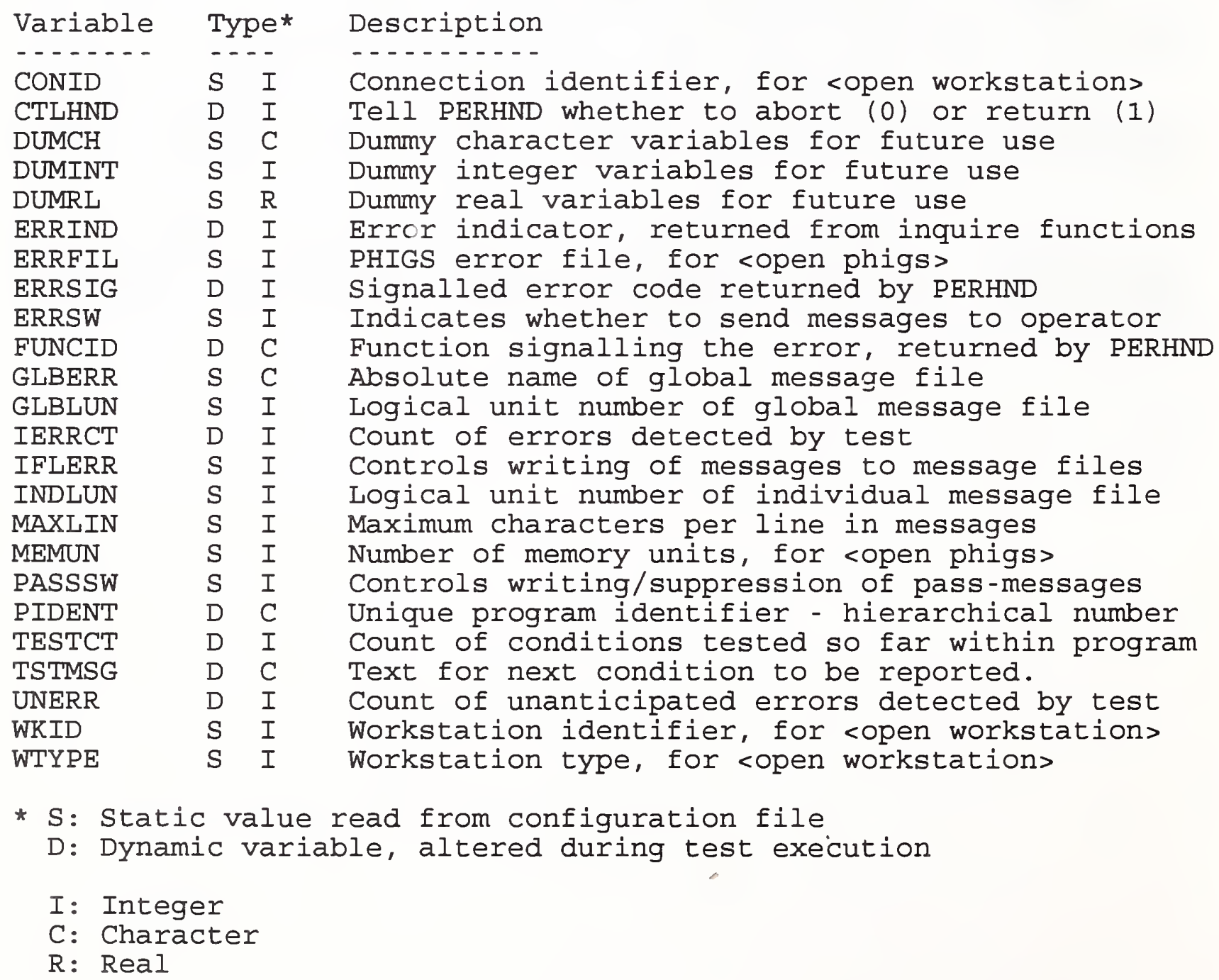


APPENDIX B

PVT TREE STRUCTURE

This appendix describes the tree structure of the PVT system. The status of each node of the tree is specified in the left column, and is one of the following:

a. Node does not contain a module. Denoted by "-".

b. Node contains a module. Denoted by a positive integer, $n$, indicating the number of programs in the module. The node contains exactly one DOC.TXT file, and n program files, numbered 1 through $\mathrm{n}$.

c. Future module(s); the node, or its descendants will contain modules in future versions of the PVT. Denoted by "f".

The second column indicates whether the node contains a local subroutine library, named SUBLIB.FOR, to be used by modules within that node's subtree. If so, an "s" appears in the column. 
0101 Opening and closing PHIGS

- 02 Manipulating the CSS

$03 \mathrm{~s} 02.01$ Creation and inquiry of CSS networks.

$10 \quad 02.01 .01$ Individual structure creation

- $\quad 02.01 .02$ Structure deletion functions

$06 \quad 02.01 .02 .01$ Individual structure deletion

14 s 02.01 .02 .02 structure network deletion

$02 \quad 02.01 .02 .03$ Global structure deletion

- s 02.01 .03 structure identification and references

03

03

03

$-$

02

03

02

05

01

$-$

02

02

07

$-$

01

01

08 02.01 .03 .01 02.01 .03 .02 Change structure identifier Change structure references 02.01 .03 .03 Change structure identifier and references 02.02 Element-level Operations

02.02 .01 Opening and closing structures

02.02 .02 Miscellaneous CSS elements

02.02 .03 Set edit mode and control of element pointer. 02.02 .04 Deletion of structure elements 02.02 .05 Copy all elements from structure 02.03 Examining CSS structures 02.03 .01 Inquiring element type and size, and content 02.03 .02 Element search

s 02.03 .03 Incremental spatial search

s 03 Archive files

03.01 Opening and closing archive files 03.02 Conflict resolution flags

s 03.03 Archiving and retrieving structures

s 03.04 Examining structures in an archive

04 s 03.05 Deleting archived structures

- s 04 Graphical Output

01 S 04.01 Primitives

$02 \quad 04.01 .01$ Polyline

$02 \quad 04.01 .02 \quad$ Polymarker

0204.01 .03 Text

0204.01 .04 Annotation text relative

$0304.01 .05 \quad$ Fill area

0304.01 .06 Fill area set

$02 \quad 04.01 .07$ Cell array

0104.01 .08 Generalized drawing primitive 


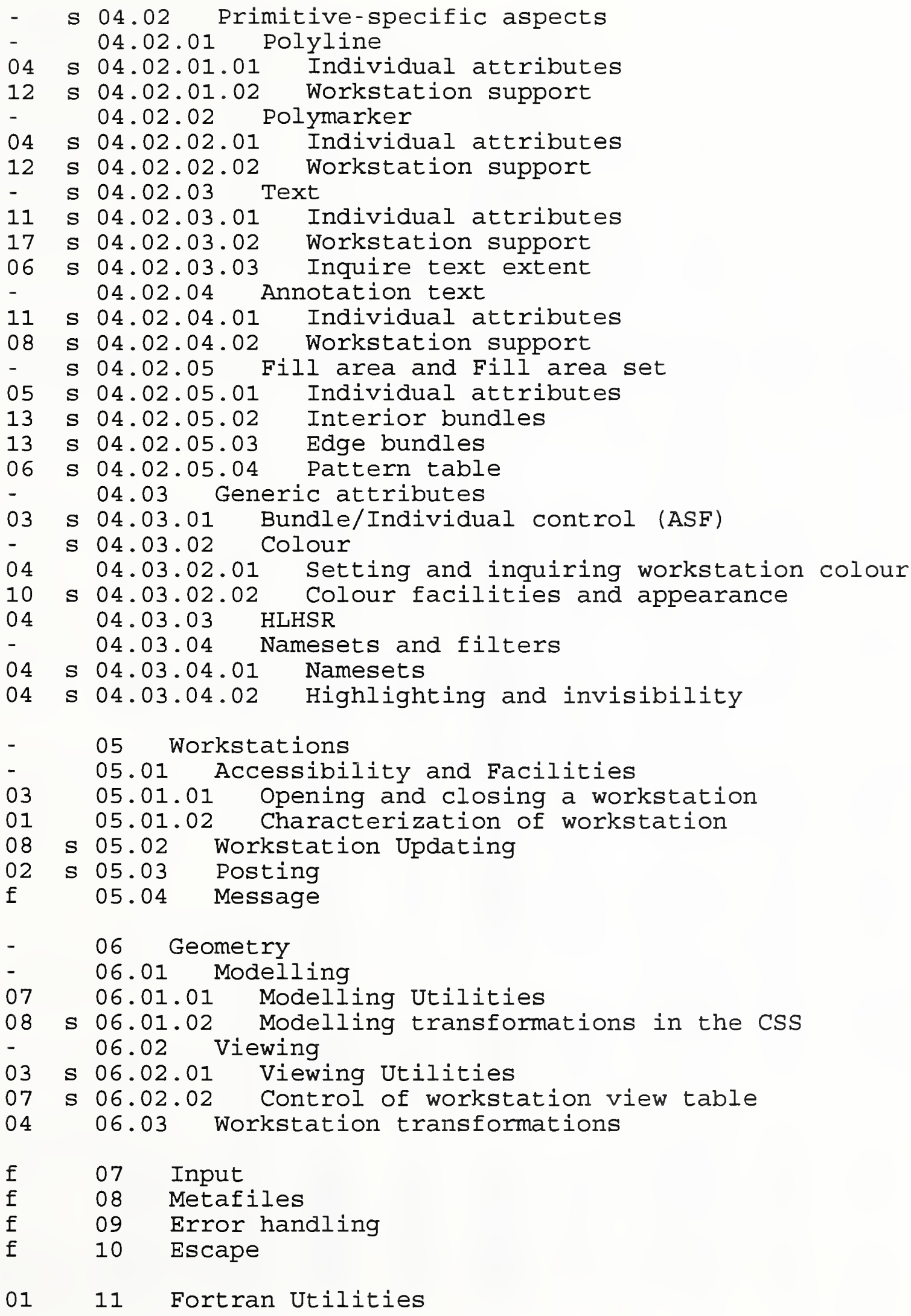




\section{APPENDIX C \\ TEST PROGRAM CHARACTERISTICS}

The following table summarizes the CHARACTERISTICS entry for all of the PVT programs. See section 2.5.6.1 for an explanation of the codes.

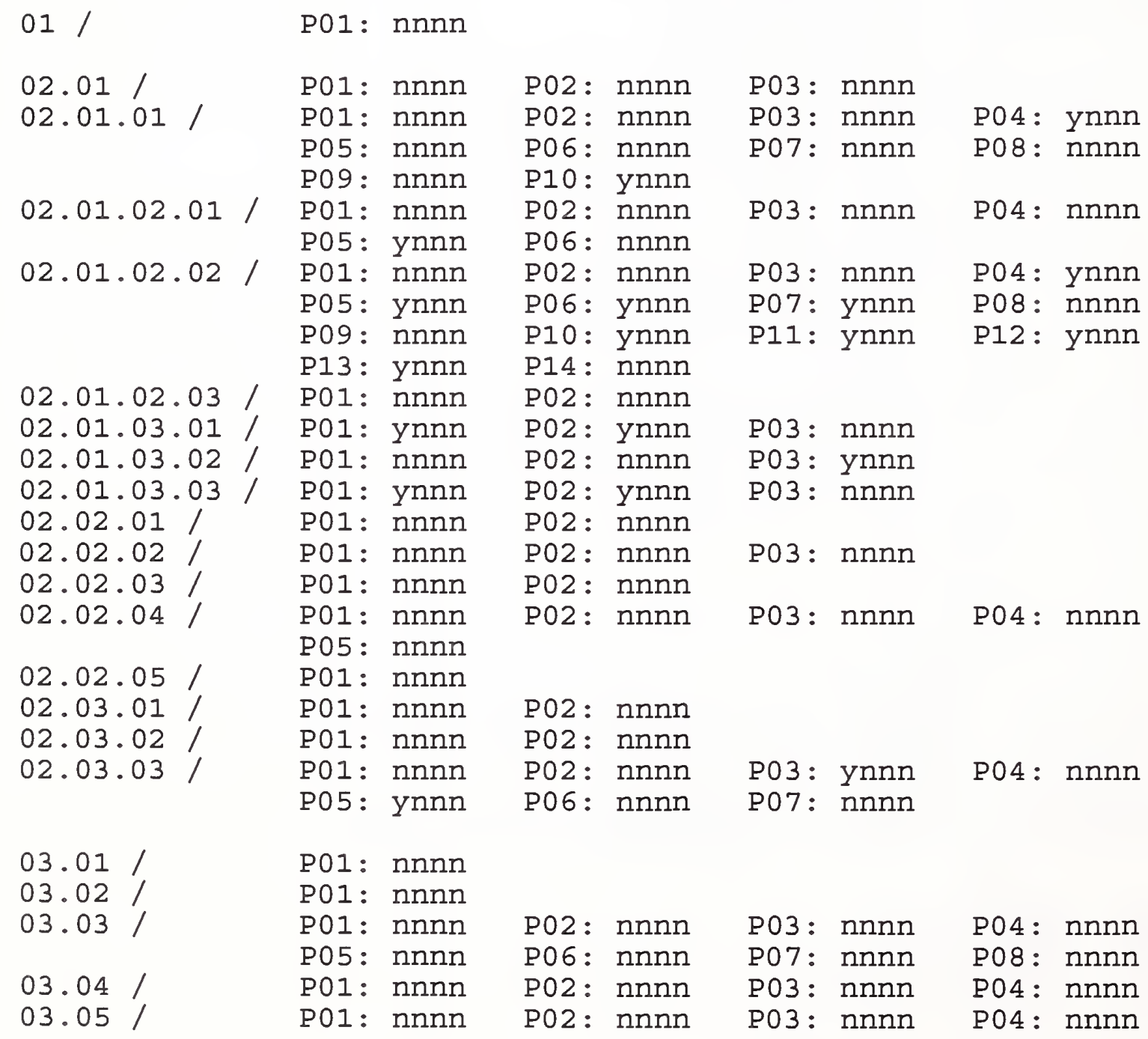




\begin{tabular}{|c|c|c|c|c|c|c|c|c|}
\hline $04.01 /$ & P01: & ynny & & & & & & \\
\hline $04.01 .01 /$ & P01: & nnnn & P02: & ynny & & & & \\
\hline $04.01 .02 /$ & P01: & nnnn & P02: & ynny & & & & \\
\hline $04.01 .03 /$ & P01: & nnnn & P02: & ynny & & & & \\
\hline $04.01 .04 \%$ & P01: & nnnn & P02: & ynny & & & & \\
\hline $04.01 .05 \%$ & P01: & nnnn & P02: & ynny & P03: & ynny & & \\
\hline $04.01 .06 \%$ & P01: & nnnn & P02: & ynny & P03: & ynny & & \\
\hline $04.01 .07 /$ & P01: & nnnn & P02 : & ynny & & & & \\
\hline $04.01 .08 /$ & P01: & ynnn & & & & & & \\
\hline $04.02 .01 .01 /$ & P01: & nnnn & P02: & ynny & P03 : & ynny & P0 4: & ynny \\
\hline \multirow{3}{*}{$04.02 .01 .02 \%$} & P01: & ynnn & P02: & ynnn & P03: & ynnn & P0 4: & ynnn \\
\hline & P05: & ynnn & P06: & ynnn & P07: & ynnn & P08: & ynnn \\
\hline & P09: & ynny & P10: & ynny & P11: & ynny & P12: & ynny \\
\hline $04.02 .02 .01 /$ & P01: & nnnn & P02: & ynny & P03: & ynny & P0 4: & ynny \\
\hline \multirow{3}{*}{$04.02 .02 .02 /$} & P01: & ynnn & P02: & ynnn & P03: & ynnn & P0 4: & ynnn \\
\hline & P05: & ynnn & P06: & ynnn & P07: & ynnn & P08: & ynnn \\
\hline & P09: & ynny & P10: & ynny & P11: & ynny & P12: & ynny \\
\hline \multirow[t]{3}{*}{$04.02 .03 .01 /$} & P01: & nnnn & P02: & ynny & P03: & ynny & P0 4: & ynny \\
\hline & P05: & ynny & P06: & ynny & P07: & ynny & P08: & ynny \\
\hline & P09: & ynny & P10: & ynny & P11: & ynny & & \\
\hline \multirow[t]{5}{*}{$04.02 .03 .02 /$} & P01: & ynnn & P02: & ynnn & P03 : & ynnn & P04: & ynnn \\
\hline & P05: & ynnn & P06: & ynnn & P07: & ynnn & P08: & ynnn \\
\hline & P09: & ynnn & P10: & ynny & P11: & ynny & P12: & ynny \\
\hline & P13: & ynny & P14: & ynny & P15: & ynny & P16: & ynny \\
\hline & P17: & ynny & & & & & & \\
\hline \multirow[t]{2}{*}{$04.02 .03 .03 /$} & P01: & ynnn & P02: & ynnn & P03 : & ynnn & P0 4: & ynnn \\
\hline & P05: & ynnn & P06: & ynny & & & & \\
\hline \multirow[t]{3}{*}{$04.02 .04 .01 /$} & P01: & nnnn & P02: & ynny & P03: & ynny & P0 4: & ynny \\
\hline & P05: & ynny & P06: & ynny & P07: & ynny & P08: & ynny \\
\hline & P09: & ynny & P10: & ynny & P11: & ynny & & \\
\hline \multirow[t]{2}{*}{$04.02 .04 .02 /$} & P01: & ynny & P02: & ynny & P03: & ynny & P04: & ynny \\
\hline & P05: & ynny & P06: & ynny & P07 : & ynny & P08 : & ynny \\
\hline \multirow{2}{*}{$04.02 .05 .01 /$} & P01: & nnnn & P02: & ynny & P03: & ynny & P04: & ynny \\
\hline & P05: & ynny & & nמng & & & & \\
\hline \multirow{3}{*}{$04.02 .05 .02 /$} & P05: & $\begin{array}{l}\text { ynnn } \\
\text { ynnn }\end{array}$ & P02: & $\begin{array}{l}\text { ynnn } \\
\text { ynnn }\end{array}$ & $\begin{array}{l}\text { P03: } \\
\text { P07: }\end{array}$ & $\begin{array}{l}\text { ynnn } \\
\text { ynnn }\end{array}$ & P04: & $\begin{array}{l}\text { ynnn } \\
\text { ynnn }\end{array}$ \\
\hline & P09: & ynnn & P10: & ynny & P11: & ynny & P12: & ynny \\
\hline & P13: & ynny & & & & & & \\
\hline \multirow[t]{4}{*}{$04.02 .05 .03 /$} & P01: & ynnn & P02: & ynnn & P03: & ynnn & P04: & ynnn \\
\hline & P05: & ynnn & P06: & ynnn & P07 : & ynnn & P08: & ynnn \\
\hline & P09: & ynnn & P10: & ynny & P11: & ynny & P12 : & ynny \\
\hline & P13: & ynny & & & & & & \\
\hline \multirow[t]{2}{*}{$04.02 .05 .04 /$} & P01: & ynnn & P02: & ynnn & P03 : & ynnn & P04: & ynnn \\
\hline & & ynnn & P06: & ynnn & & & & \\
\hline $04.03 .01 /$ & P01: & nnnn & P02: & ynny & P03: & ynny & & \\
\hline $04.03 .02 .01 /$ & P01: & ynnn & P02: & ynnn & P03: & ynnn & P04: & ynny \\
\hline \multirow[t]{3}{*}{$04.03 .02 .02 /$} & P01: & ynnn & P02: & ynnn & P03: & ynnn & P04: & ynnn \\
\hline & P05: & ynnn & P06: & ynny & P07 : & ynny & P08 : & ynny \\
\hline & P09: & ynny & P10 : & ynny & & & & \\
\hline $04.03 .03 /$ & P01: & ynnn & P02: & ynnn & P03: & ynnn & P04: & ynnn \\
\hline 04.03 .04 .01 & P01: & nnnn & P02: & nnnn & P03: & nnnn & P04: & ynny \\
\hline $04.03 .04 .02 /$ & P01: & ynnn & P02: & ynnn & P03: & ynny & P04: & ynny \\
\hline
\end{tabular}




\begin{tabular}{|c|c|c|c|c|c|c|c|c|}
\hline $\begin{array}{l}05.01 .01 / \\
05.01 .02 /\end{array}$ & $\begin{array}{l}\text { P01: } \\
\text { P01: }\end{array}$ & $\begin{array}{l}\text { nnnn } \\
\text { nnnn }\end{array}$ & P02: & nnnn & P03: & nnnn & & \\
\hline $05.02 /$ & $\begin{array}{l}\text { P01: } \\
\text { P05: }\end{array}$ & ynnn & P02: & ynnn & P03: & ynnn & P04: & ynnn \\
\hline $05.03 /$ & P01: & nnnn & P02: & ynnn & 18 & & & \\
\hline $06.01 .01 /$ & $\begin{array}{l}\text { P01: } \\
\text { P05: }\end{array}$ & $\begin{array}{l}\text { nnnn } \\
\text { nnnn }\end{array}$ & $\begin{array}{l}\text { P02: } \\
\text { P06: }\end{array}$ & $\begin{array}{l}\text { nnnn } \\
\text { nnnn }\end{array}$ & $\begin{array}{l}\text { P03: } \\
\text { P07: }\end{array}$ & $\begin{array}{l}\text { nnnn } \\
\text { nnnn }\end{array}$ & P04: & $n n r$ \\
\hline $06.01 .02 /$ & $\begin{array}{l}\text { P01: } \\
\text { P05: }\end{array}$ & $\begin{array}{l}\text { nnnn } \\
\text { nnnn }\end{array}$ & $\begin{array}{l}\text { P02 : } \\
\text { P06: }\end{array}$ & $\begin{array}{l}\text { nnnn } \\
\text { nnnn }\end{array}$ & $\begin{array}{l}\text { P03: } \\
\text { P07: }\end{array}$ & $\begin{array}{l}\text { nnnn } \\
\text { nnnn }\end{array}$ & $\begin{array}{l}\text { P04: } \\
\text { P08: }\end{array}$ & $\begin{array}{l}\text { nnn } \\
n n n\end{array}$ \\
\hline $06.02 .01 /$ & POI: & nnnn & P02: & nnnn & P03: & nnnn & & \\
\hline $06.02 .02 /$ & $\begin{array}{l}\text { P01: } \\
\text { P05: }\end{array}$ & $\begin{array}{l}\text { ynnn } \\
\text { ynnn }\end{array}$ & $\begin{array}{l}\text { P02 : } \\
\text { P06: }\end{array}$ & $\begin{array}{l}\text { ynnn } \\
\text { ynnn }\end{array}$ & $\begin{array}{l}\text { P03: } \\
\text { P07: }\end{array}$ & $\begin{array}{l}\text { ynnn } \\
\text { nnnn }\end{array}$ & P04: & \\
\hline $06.03 /$ & POI: & ynnn & P02: & ynnn & P03: & ynnn & P04: & \\
\hline
\end{tabular}




\section{APPENDIX D \\ DICTIONARY OF SUBROUTINES AND FUNCTIONS}

This table lists all subroutines and functions in the PVT, in alphabetical order. The two columns following each function name indicate whether it is a subroutine or function, and its location. For example, ACTST is a subroutine in PVT 02 01 03 SUBLIB. FOR, APPEQ is a logical function in PVT SUBLIB.FOR, and ARRTP3 is a subroutine in PVT TRANS_SUBLIB.FOR.

$\begin{array}{ll}\text { abend } & \text { SUBROUT GL } \\ \text { altsiz } & \text { SUBROUT 04.02 } \\ \text { arcpth } & \text { SUBROUT 03.04 } \\ \text { arreq } & \text { LOGC_FN 04.02.05.04 } \\ \text { asfint } & \text { SUBROUT } 04.03 .01 \\ \text { avarnm } & \text { SUBROUT 03 } \\ \text { brdmsg } & \text { SUBROUT GL } \\ \text { chkelp } & \text { SUBROUT GL } \\ \text { chkinq } & \text { SUBROUT GL } \\ \text { chkpst } & \text { SUBROUT 05.03 } \\ \text { chtxrc } & \text { SUBROUT 04.02.03.03 } \\ \text { coleql } & \text { LOGC_FN 04.03.02.02 } \\ \text { colpch } & \text { SUBROUT 04.03.02.02 } \\ \text { comtok } & \text { SUBROUT 06.01.02 } \\ \text { crossp } & \text { SUBROUT TR } \\ \text { cssids } & \text { LOGC_FN 03.03 } \\ \text { curnms } & \text { SUBROUT 04.03.04.01 } \\ \text { dchflv } & \text { LOGC_FN GL } \\ \text { dchpf } & \text { SUBROUT GL } \\ \text { deblnk } & \text { SUBROUT GL } \\ \text { dilist } & \text { SUBROUT GL } \\ \text { disedb } & \text { SUBROUT 04.02.05.03 } \\ \text { displb } & \text { SUBROUT 04.02.01.02 } \\ \text { distxb } & \text { SUBROUT 04.02 } \\ \text { dlstpf } & \text { SUBROUT GL } \\ \text { dotprd } & \text { REAL_FN TR } \\ \text { drlval } & \text { SUBROUT GL } \\ \text { drwedb } & \text { SUBROUT 04.02.05.03 } \\ \text { drwplb } & \text { SUBROUT 04.02.01.02 } \\ \text { drwrec } & \text { SUBROUT 04 } \\ \text { dsanst } & \text { SUBROUT 04.02.04.01 } \\ \text { dyn } & \text { LOGC_FN GL } \\ & \\ & \end{array}$

$\begin{array}{ll}\text { actst } & \text { SUBROUT 02.01.03 } \\ \text { appeq } & \text { LOGC_FN GL } \\ \text { arcpts } & \text { SUBROUT 04 } \\ \text { arrtp3 } & \text { SUBROUT TR } \\ \text { asfset } & \text { SUBROUT } 04.03 .01 \\ \text { basbot } & \text { REAL_FN } 04.02 \\ \text { ceilng } & \text { INTG_FN TR } \\ \text { chkfnt } & \text { SUBROUT 04.02 } \\ \text { chkmcV } & \text { SUBROUT 06.01.02 } \\ \text { chmono } & \text { SUBROUT 04.02.03.03 } \\ \text { cldiag } & \text { SUBROUT 04.02.05.01 } \\ \text { colnam } & \text { SUBROUT 04.03.02 } \\ \text { coltrn } & \text { SUBROUT 04.03.02.02 } \\ \text { cpyiar } & \text { SUBROUT GL } \\ \text { csseq } & \text { LOGC_FN GL } \\ \text { csspth } & \text { SUBROUT GL } \\ \text { dchfl } & \text { LOGC_FN GL } \\ \text { dchoic } & \text { SUBROUT GL } \\ \text { dchpfv } & \text { SUBROUT GL } \\ \text { delcss } & \text { SUBROUT 02.01.02.02 } \\ \text { discol } & \text { SUBROUT 04 } \\ \text { disinb } & \text { SUBROUT 04.02.05.02 } \\ \text { dispmb } & \text { SUBROUT 04.02.02.02 } \\ \text { dline } & \text { SUBROUT GL } \\ \text { doimod } & \text { SUBROUT 05.02 } \\ \text { drbued } & \text { SUBROUT 04.02.05.03 } \\ \text { drwatx } & \text { SUBROUT 04.02.04.02 } \\ \text { drwint } & \text { SUBROUT 04.02.05.02 } \\ \text { drwpmb } & \text { SUBROUT 04.02.02.02 } \\ \text { drwtxt } & \text { SUBROUT 04.02.03.02 } \\ \text { dstrng } & \text { SUBROUT GL } \\ \text { dynpf } & \text { SUBROUT GL } \\ & \end{array}$




$\begin{array}{ll}\text { ebltm } & \text { SUBROUT TR } \\ \text { ecom } & \text { SUBROUT TR } \\ \text { ecotm } & \text { SUBROUT TR } \\ \text { eevmm } & \text { SUBROUT TR } \\ \text { eevom3 } & \text { SUBROUT TR } \\ \text { elstr } & \text { SUBROUT 02.01.03 } \\ \text { ero } & \text { SUBROUT TR } \\ \text { eroy } & \text { SUBROUT TR } \\ \text { errctl } & \text { SUBROUT GL } \\ \text { esc3 } & \text { SUBROUT TR } \\ \text { etp3 } & \text { SUBROUT TR } \\ \text { etr3 } & \text { SUBROUT TR } \\ \text { expppl } & \text { SUBROUT 04.02.01.01 } \\ \text { fail } & \text { SUBROUT GL } \\ \text { fitpar } & \text { SUBROUT GL } \\ \text { fpav1 } & \text { LOGC FN 04.02 } \\ \text { gtroot } & \text { SUBROUT GL } \\ \text { hueang } & \text { SUBROUT 04.03.02.02 } \\ \text { iacmsg } & \text { SUBROUT GL } \\ \text { iareq1 } & \text { LOGC FN GL } \\ \text { idmat } & \text { SUBROUT TR } \\ \text { inarea } & \text { INTG FN TR } \\ \text { initgl } & \text { SUBROUT GL } \\ \text { inoutl } & \text { SUBROUT 04.02.03 } \\ \text { intsty } & \text { LOGC FN 04 } \\ \text { ispths } & \text { SUBROUT TR } \\ \text { issdis } & \text { SUBROUT TR } \\ \text { issgeo } & \text { SUBROUT 02.03.03 } \\ \text { itrim } & \text { INTG_FN GL } \\ \text { leadnb } & \text { INTG FN GL } \\ \text { lintpt } & \text { SUBROUT TR } \\ \text { locat2 } & \text { SUBROUT 04.02.04.01 } \\ \text { locint } & \text { SUBROUT 04 } \\ \text { locppm } & \text { SUBROUT GL } \\ \text { loctri } & \text { SUBROUT 04 } \\ \text { loctx2 } & \text { SUBROUT 04.02.03.01 } \\ \text { matmul } & \text { SUBROUT TR } \\ \text { modsep } & \text { REAL FN 04.03.02.02 } \\ \text { ncmsg } & \text { SUBROUT GL } \\ \text { ndmw } & \text { SUBROUT 04.02.02.01 } \\ \text { ngtxat } & \text { SUBROUT 04.02 } \\ \text { nmsprm } & \text { SUBROUT 04.03.04.02 } \\ \text { opco } & \text { SUBROUT GL } \\ \text { opcomt } & \text { SUBROUT GL } \\ \text { opmsg } & \text { SUBROUT GL } \\ \text { opyn } & \text { SUBROUT GL } \\ \text { parlin } & \text { SUBROUT 04.02.05.01 } \\ \text { pass } & \text { SUBROUT GL } \\ \text { patrec } & \text { SUBROUT 04.02.05 } \\ \text { perveC } & \text { SUBROUT TR } \\ \text { picchg } & \text { SUBROUT 05.02 } \\ \text { plattr } & \text { SUBROUT 04.02.01.01 } \\ \text { prpfnt } & \text { SUBROUT 04.02 } \\ \text { prsint } & \text { SUBROUT GL } \\ & \end{array}$

\begin{tabular}{|c|c|c|}
\hline ebltm3 & SUBROUT & TR \\
\hline ecom3 & SUBROUT & TR \\
\hline ecotm3 & SUBROUT & TR \\
\hline eevom & SUBROUT & TR \\
\hline lgprm & SUBROUT & 04.03 .04 .02 \\
\hline ndit & SUBROUT & GL \\
\hline erox & SUBROUT & TR \\
\hline eroz & SUBROUT & TR \\
\hline esc & SUBROUT & TR \\
\hline etp & SUBROUT & TR \\
\hline etr & SUBROUT & TR \\
\hline $\exp 34$ & SUBROUT & TR \\
\hline expppm & SUBROUT & 04.02 .02 .01 \\
\hline filrec & SUBROUT & 04.01 \\
\hline fltran & SUBROUT & 02.03 .03 \\
\hline geotxt & SUBROUT & 04.02 .03 \\
\hline hlfbox & SUBROUT & 04.02 \\
\hline iacans & SUBROUT & GL \\
\hline iareq & LOGC_FN & GL \\
\hline iarfnd & INTG_FN & GL \\
\hline ifpf & SUBRŌUT & GL \\
\hline iniari & SUBROUT & GL \\
\hline inmsg & SUBROUT & GL \\
\hline intinh & SUBROUT & 04.03 .01 \\
\hline invol & SUBROUT & $\mathrm{TR}$ \\
\hline issab & SUBROUT & $\mathrm{TR}$ \\
\hline issflt & SUBROUT & 02.03 .03 \\
\hline isspf & SUBROUT & 02.03 .03 \\
\hline Ibedty & SUBROUT & 04.02 .05 .03 \\
\hline linbrk & SUBROUT & GL \\
\hline locans & SUBROUT & 04.02 .04 .01 \\
\hline locatx & SUBROUT & 04 \\
\hline locppl & SUBROUT & GL \\
\hline locrec & SUBROUT & 04.02 .05 \\
\hline loct $x 1$ & SUBROUT & 04.02 .03 .01 \\
\hline loctxt & SUBROUT & 04 \\
\hline $\bmod 1$ & INTG_FN & TR \\
\hline multws & SUBRŌUT & GL \\
\hline ndlw & SUBROUT & 04.02 .01 .01 \\
\hline newstr & SUBROUT & GL \\
\hline nmscol & SUBROUT & 04.03 .04 .02 \\
\hline numlab & SUBROUT & GL \\
\hline opcofl & SUBROUT & GL \\
\hline opfail & SUBROUT & GL \\
\hline opmsgw & SUBROUT & GL \\
\hline parcof & SUBROUT & $\mathrm{TR}$ \\
\hline parpt & SUBROUT & 04.02 .05 .01 \\
\hline patavl & LOGC_FN & 04.02 .05 \\
\hline perhnd & SUBROUUT & GL \\
\hline$p f$ & SUBROUT & GL \\
\hline picenv & SUBROUT & 05.02 \\
\hline pmattr & SUBROUT & 04.02 .02 .01 \\
\hline prpv1 & SUBROUT & $\mathrm{TR}$ \\
\hline prsrl & SUBROUT & GL \\
\hline
\end{tabular}




$\begin{array}{ll}\text { prsuin } & \text { SUBROUT GL } \\ \text { pt2cof } & \text { SUBROUT TR } \\ \text { pthseq } & \text { SUBROUT 02.01 } \\ \text { ptplds } & \text { SUBROUT TR } \\ \text { ptspl } & \text { SUBROUT TR } \\ \text { ranmkt } & \text { INTG_FN 04.02.02.01 } \\ \text { red43 } & \text { SUBROUT TR } \\ \text { rnlshf } & \text { SUBROUT GL } \\ \text { rnd01 } & \text { REAL_FN GL } \\ \text { rndrl } & \text { REAL_FN GL } \\ \text { rnset } & \text { SUBROUT GL } \\ \text { scapar } & \text { SUBROUT GL } \\ \text { setasf } & \text { SUBROUT GL } \\ \text { setdlg } & \text { SUBROUT GL } \\ \text { setfil } & \text { SUBROUT 02.03.03 } \\ \text { setis } & \text { LOGC_FN GL } \\ \text { setpcl } & \text { SUBROUT 05.02 } \\ \text { setrvs } & \text { SUBROUT GL } \\ \text { setsvr } & \text { SUBROUT 05.02 } \\ \text { setvs } & \text { SUBROUT GL } \\ \text { showew } & \text { SUBROUT 04.02.05.01 } \\ \text { showmw } & \text { SUBROUT 04.02.02.01 } \\ \text { shpmbw } & \text { SUBROUT 04.02.02.02 } \\ \text { sigtst } & \text { SUBROUT GL } \\ \text { sqgrmk } & \text { SUBROUT 04.03.02 } \\ \text { srtrar } & \text { SUBROUT GL } \\ \text { strcon } & \text { LOGC_FN GL } \\ \text { surfok } & \text { LOGC_FN 05.02 } \\ \text { svrok } & \text { LOGCEN 05.02 } \\ \text { tcscir } & \text { SUBROUT 02.01.03 } \\ \text { trnseq } & \text { LOGC_FN TR } \\ \text { tschup } & \text { SUBROUT 04.02.03 } \\ \text { tstcid } & \text { SUBROUT 02.01.03 } \\ \text { tstcor } & \text { SUBROUT 06.02.01 } \\ \text { tsthue } & \text { INTG_FN 04.03.02.02 } \\ \text { tstprj } & \text { SUBROUT 06.02.01 } \\ \text { tx2dex } & \text { SUBROUT 02.03.03 } \\ \text { txexal } & \text { SUBROUT 04.02 } \\ \text { undcmd } & \text { LOGC_FN 04.03.02.02 } \\ \text { unrep } & \text { SUBROUT 04.02 } \\ \text { vec1 } & \text { SUBROUT TR } \\ \text { vecl } & \text { REAL_FN TR } \\ \text { vislab } & \text { SUBROUT GL } \\ \text { win6 } & \text { SUBROUT GL } \\ \text { xfinh } & \text { SUBROUT 04 } \\ \text { ylocel } & \text { REAL_FN GL } \\ \text { zval } & \text { REAL_FN 02.03.03 } \\ & \end{array}$

\begin{tabular}{|c|c|c|}
\hline pstctl & SUBROUT & GL \\
\hline pt3pl & SUBROUT & TR \\
\hline ptlnds & SUBROUT & TR \\
\hline ptregd & REAL FN & TR \\
\hline ranlst & INTG $^{-} F N$ & 04.02 .01 .01 \\
\hline rareq & LOGC_FN & GL \\
\hline retopn & SUBRŌUT & 03.03 \\
\hline rnbset & SUBROUT & GL \\
\hline rndint & INTG_FN & GL \\
\hline rnperm & SUBRŌUT & GL \\
\hline satan2 & REAL_FN & TR \\
\hline set $2 d$ & SUBRŌUT & GL \\
\hline setdif & SUBROUT & 03.05 \\
\hline eteq & LOGC FN & GL \\
\hline ethue & SUBRŌUT & 04.03 .02 .02 \\
\hline etmsg & SUBROUT & GL \\
\hline setpst & SUBROUT & 05.03 \\
\hline setstr & SUBROUT & GL \\
\hline setval & SUBROUT & GL \\
\hline shedbw & SUBROUT & 04.02 .05 .03 \\
\hline showlw & SUBROUT & 04.02 .01 .01 \\
\hline shplbw & SUBROUT & 04.02 .01 .02 \\
\hline igmsg & SUBROUT & GL \\
\hline simark & SUBROUT & 04.02 \\
\hline srtiar & SUBROUT & GL \\
\hline stdcss & SUBROUT & GL \\
\hline ubfnt & SUBROUT & 04.02 \\
\hline vrexp & INTG FN & 05.02 \\
\hline tcscid & SUBRŌUT & 02.01 .03 \\
\hline tranhs & SUBROUT & 06.01 .02 \\
\hline tsanup & SUBROUT & 04.02 .04 .01 \\
\hline tstasc & SUBROUT & 04.02 \\
\hline tstcir & SUBROUT & 02.01 .03 \\
\hline tsthlf & SUBROUT & 04.03 .04 .02 \\
\hline tstivf & SUBROUT & 04.03 .04 .02 \\
\hline tstvip & SUBROUT & 06.02 .02 \\
\hline txcomp & SUBROUT & 04.02 .03 .03 \\
\hline xpcup & SUBROUT & 04.02 \\
\hline amsg & SUBROUT & GL \\
\hline $\operatorname{arfnt}$ & SUBROUT & 04.02 \\
\hline ecang & REAL_FN & $\mathrm{TR}$ \\
\hline $\mathrm{ecl} 2$ & REAL_FN & TR \\
\hline$n p c$ & SUBRŌUT & 04 \\
\hline indup & SUBROUT & GL \\
\hline oopph & SUBROUT & GL \\
\hline oos 8 & REAL FN & 04.03 .04 .02 \\
\hline
\end{tabular}




\section{APPENDIX E \\ EXAMPLES OF COMMAND PROCEDURES}

Below are two examples of a compile, link, and execute procedure for PVT programs. The first is for the VAX/VMS system, the second for UNIX. These are for illustrative purposes only.

VAX/VMS

\$! Procedure to invoke DecPHIGs and access PVT libraries

$\$$ set nover

$\$$ define sys\$input sys\$command

$\$$ locsub = " " ! this will be string with names

$\$$

$\$$ curdir = f\$directory () ! name of current directory

$\$$ pvtloc = f\$locate ("PVT.", curdir) ! PVT. is root level

\$ dirlen = f\$length (curdir) ! length of current directory name

$\$$ numlev $=($ dirlen - pvtloc -4$) / 3$ ! number of levels of PVT

$\$$ backup $=0$

$\$$ ! loop up directory levels till root

\$ sub_loop:

$\$$

$\$$ seekfile $="["+$ f\$extract $(1$, backup, $"-\ldots . .-1 ")+$ + $]$ sublib"

$\$$ ! gotfile is null string if no sublib at this level

$\$$ gotfile = f\$search (seekfile + ".olb")

\$ if (gotfile .nes. " ") then -

locsub = locsub + seekfile + "/lib," ! tack on next name

$\$$ backup $=$ backup +1

\$ if (backup.lt. numlev) then goto sub_loop

\$ set ver

\$ fort/warn=all/standard 'p1' ! compile test program

\$ link 'p1', 'locsub' [USER.PVT] TRANS_SUBLIB/LIB, -

$\$ \operatorname{run}$ 'pl'

[USER.PVT] SUBLIB/LIB, SYS\$LIBRARY:PHIGS\$FOR_BND/LIB 
\#!/bin/csh

\# This script:

\# Determines the language of the input file (FTN or C)

\# Compiles the source to object code

\# Searches up the directory tree for libraries (sublib.a).

\#

$\#$ Stops searching at a directory called "v2" or root. Links the executable code with all libraries found from topdir down.

Executes the program.

If any step fails, the script will exit before proceeding.

\# Test searching of libraries by invoking with an argument

\# of "hi". See usage at bottom for usage instructions.

\# USER CONFIGURATION VARIABLES

\# Change libext if libraries have different extensions. set libext $=$ 'sublib.a'

set trans_lib $=$ '/home/pvt/v2/trans_sublib.a' set global_lib $=$ ' /home/pvt/v2/sublib.a'

\# Set PRE_PHIGS_LIB to point to your Phigs libraries. This is \# the first líbrary listed in the link command.

set PRE_PHIGS_LIB = " "

\# set POST_PHIGS LIB to your phigs library. This is listed

\# LAST iñ the İink command.

set POST_PHIGS_LIB = "-lphigs77-lphigs-lxgl-1X11-lm"

set libs $=" "$

if ( $\$ \#$ argv $==0$ ) goto usage

if $(\$ 1=="-v ")$ then

set echocmd = " " shift

endif

if ( $\$ \#$ argv < 1 ) goto usage

if ( $-r$ \$argv.f ) then \# Fortran source

set $\mathrm{CC}=$ "f77-O - u $-\mathrm{xl} " \mathrm{EXT}=$. f

else if ( $-r$ \$argv.c ) then \#C source

set $\mathrm{CC}=\mathrm{CC} \quad \mathrm{EXT}=. \mathrm{C}$

else if ( \$argv != "hi") then

\# if ext. not known \& not testing, exit.

echo $\$\{0\}$ : Could not read \$argv.f or $\backslash$

exit

sargv.c\! Fix and try again.

endif 
if ( Sargv != "hi") then

if ( $\$$ ?echocmd) echo \$CC - c \$argv\$EXT

\$CC - C \$argv\$EXT \# compile to .o

if ( status != 0 ) then \# Bogus compilation?

echo $\$\{0\}$ : Fix compilation errors in $\backslash$

\$argv\$EXT and try again.

endif exit

endif

if ( $\$$ ?echocmd) echo $-\mathrm{n} \$\{0\}$ : Searching for library archives...

set curdir = 'pwd'

while ( \$curdir != " ")

if ( curdir:t == "v2") break \# just processed v2 dir?

/bin/ls \$curdir/\$libext >\&/dev/null \# avoid "no match" msgs

if ( $\$$ status $==0$ ) then \# found some!

endif

set libs $=(\$ l i b s$ ' $/$ bin/ls $\$$ curdir/\$libext')

end

set curdir $=$ \$curdir:h \# leave head (chop tail)

if ( \$?echocmd) echo done.

if ( argv == "hi" || \$?echocmd) then

echo $\$\{0\}$ : Libraries found:

echo \$libs

endif

exit

if ( \$?echocmd) echo \$CC \$PRE_PHIGS_LIB \$argv.0 ।

\$libs \$global_Iib \$POST_PHIGS_LIB - o \$argv

\$CC \$PRE_PHIGS_LIB \$argV.O

\$libs \$trañs_lib \$global_lib \$POST_PHIGS_LIB - o \$argv

if ( $\$$ status $==\overline{0}$ ) then - \# linkéd oK

if (\$?echocmd) echo./\$argv

else

. / \$argv \# execute

echo $\$\{0\}$ : Fix link errors and try again.

endif exit

\#\#\#\#\#\#\#\#\#\#\#\#\#\#\#

usage:

echo Usage: $\$ 0$ ' $[-\mathrm{v}]$ file'

echo Note: file has no extension, but file.f or file.c exists. echo ' $-\mathrm{v}$ ' option will echo commands before they are executed.

exit 


\section{APPENDIX F}

\section{SOFTWARE METRICS}

The size of the PVT system may be measured in several ways. The following table presents some size metrics for version 2.0.

$\begin{array}{llr}\text { number of modules } & : & 60 \\ \text { number of test programs } & : & 300 \\ \text { number of lines in test programs } & : 69,910 \\ \text { number of subroutine libraries } & : & 38 \\ \text { number of subroutines/functions } & : 65 \\ \text { number of lines in subroutines/functions } & : 18,757 \\ \text { number of lines in system utilities } & : & 632 \\ \text { number of lines in module documentation } & : 57,100 \\ \text { number of lines in system documentation } & : 9,991 \\ \text { number of SRs (semantic requirements) } & : 1,221 \\ \text { number of TCs (test cases) } & : & 1,723 \\ \text { staff-months of effort } & : & 74 \\ \text { (design, code, documentation) } & \end{array}$




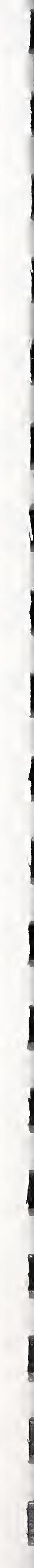


APPENDIX G

FUNCTION CROSS-REFERENCE

\#F 001 <open phigs>:

01/SR01 02.01.01/SR0 8 02.02.03/SR05 03.01/SR04 03.02/SR01

$05.01 .01 /$ SR01 $05.01 .01 /$ SR02

\#F $002<$ close phigs>:

01/SR02

\#F 003 <open workstation>:

$04.02 .01 .02 /$ SR24 $04.02 .01 .02 /$ SR2 $504.02 .02 .02 /$ SR2 4

$04.02 .02 .02 /$ SR25 $04.02 .03 .02 /$ SR2 $4 \quad 04.02 .03 .02 /$ SR2 5

$04.02 .04 .02 /$ SR09 $04.02 .05 .02 /$ SR2 $504.02 .05 .02 /$ SR2 6

$04.02 .05 .03 /$ SR24 $04.02 .05 .03 /$ SR2 $504.02 .05 .04 / S R 16$

$04.02 .05 .04 /$ SR17 $04.03 .02 .02 /$ SR13 $04.03 .02 .02 /$ SR14

$04.03 .02 .02 /$ SR20 $04.03 .04 .02 /$ SR03 $04.03 .04 .02 /$ SR0 $705.01 .01 /$ SR0 6

$05.01 .01 /$ SR0 8 05.01.01/SR11 05.01.02/SR0 $605.02 /$ SR0 $805.02 /$ SR0 9

$05.03 /$ SR10 $06.02 .02 /$ SR09 $06.02 .02 /$ SR10 $06.02 .02 /$ SR11

$06.02 .02 /$ SR19 $06.03 /$ SR05

\#F $004<$ close workstation>:

$05.01 .01 /$ SR12 $05.03 /$ SR11

\#F 005 <redraw all structures >

$05.02 /$ SR16

\#F 006 <update workstation>:

$05.02 /$ SR17 $05.02 /$ SR18

\#F 007 <set display update state>:

$05.02 /$ SR01 05.02/SR02 05.02/SR03 05.02/SR08 $05.02 /$ SR22 $05.02 / S R 23$

$05.02 /$ SR2 $405.02 /$ SR2 $505.02 /$ SR2 $605.02 / S R 2705.02 / S R 2805.02 / S R 29$

$05.02 /$ SR $3105.02 /$ SR3 2

\#F 009 <polyline $3>$ :

$02.03 .03 /$ SR0 $62.03 .03 /$ SR10 $02.03 .03 /$ SR12 $04.01 .01 /$ SR01

$04.01 .01 /$ SR0 $704.01 .03 /$ SR09 $04.01 .03 /$ SR $1104.03 .04 .01 /$ SR 15

$05.02 /$ SR19 $05.02 /$ SR20 $05.02 /$ SR21 $06.01 .02 /$ SR $1706.01 .02 / S R 42$

\#F $010<$ polylines: 
$02.03 .03 /$ SR06 $02.03 .03 /$ SR10 $02.03 .03 /$ SR12 $04.01 .01 /$ SR0 4 $04.01 .01 /$ SR07 $04.01 .03 /$ SR09 $04.01 .03 /$ SR10 $04.03 .04 .01 /$ SR15 $05.02 /$ SR19 $05.02 /$ SR20 $05.02 /$ SR21 $06.01 .02 /$ SR17 $06.01 .02 / S R 42$

\#F 011 <polymarker 3>: $02.03 .03 /$ SR0 $62.03 .03 /$ SR10 $02.03 .03 /$ SR11 $04.01 .02 /$ SR01 $04.01 .02 /$ SR0 $704.03 .04 .01 /$ SR15 $05.02 /$ SR19 $05.02 /$ SR20 $05.02 / S R 21$ $06.01 .02 /$ SR17 $06.01 .02 /$ SR 42

\#F 012 <polymarker>:

$02.03 .03 /$ SR0 $62.03 .03 /$ SR10 $02.03 .03 /$ SR11 $04.01 /$ SR01

$04.01 .02 /$ SR04 04.01.02/SR07 04.03.04.01/SR15 05.02/SR19

$05.02 /$ SR20 05.02/SR21 $06.01 .02 /$ SR17 $06.01 .02 / S R 42$

\#F $013<$ text $3>$ :

$02.03 .03 /$ SR0 $62.03 .03 /$ SR10 $02.03 .03 /$ SR15 $04.01 .03 /$ SR01

$04.02 .03 .01 /$ SR01 $04.02 .03 .01 /$ SR02 $04.03 .04 .01 /$ SR15 $05.02 /$ SR19

$05.02 /$ SR20 05.02/SR21 $06.01 .02 /$ SR17 $06.01 .02 / S R 42$

\#F $014<$ <text>:

$02.03 .03 /$ SR0 $62.03 .03 /$ SR10 $02.03 .03 /$ SR15 $04.01 .03 /$ SR0 4 $04.02 .03 .01 /$ SR01 $04.02 .03 .01 /$ SR02 $04.03 .04 .01 /$ SR15 $05.02 /$ SR19 $05.02 / S R 2005.02 / S R 2106.01 .02 / S R 1706.01 .02 / S R 42$

\#F 015 cannotation text relative $3>$ : $02.03 .03 /$ SR0 $62.03 .03 /$ SR10 $02.03 .03 /$ SR16 $04.01 .04 /$ SR01 04.01.04/SR07 04.01.04/SR09 04.02.04.01/SR01 04.02.04.01/SR02 $04.03 .04 .01 /$ SR15 $05.02 /$ SR19 $05.02 /$ SR20 $05.02 /$ SR2 $106.01 .02 /$ SR 17 $06.01 .02 /$ SR 42

\#F 016 cannotation text relatives:

$02.03 .03 /$ SR0 $602.03 .03 /$ SR10 $02.03 .03 /$ SR16 $04.01 .04 /$ SR0 4 $04.01 .04 /$ SR07 04.01.04/SR08 04.01.04/SR09 $04.02 .04 .01 /$ SR01 $04.02 .04 .01 /$ SR02 $04.03 .04 .01 /$ SR15 $05.02 /$ SR19 $05.02 /$ SR2 0 $05.02 /$ SR21 $06.01 .02 /$ SR17 $06.01 .02 /$ SR4 2

\#F $017<$ fill area $3>$ :

$02.03 .03 /$ SR06 $02.03 .03 /$ SR10 $02.03 .03 /$ SR13 $04.01 .05 /$ SR01 $04.01 .05 /$ SR0 $704.01 .05 /$ SR08 $04.03 .04 .01 /$ SR15 $05.02 /$ SR19 $05.02 /$ SR20 05.02/SR21 06.01.02/SR17 06.01.02/SR42

\#F $018<$ fill area>:

$02.03 .03 /$ SR0 $62.03 .03 /$ SR10 $02.03 .03 /$ SR13 $04.01 .05 /$ SR0 4 $04.01 .05 /$ SR0 $704.01 .05 /$ SR08 $04.03 .04 .01 /$ SR15 $05.02 /$ SR19 $05.02 /$ SR20 05.02/SR21 06.01.02/SR17 06.01.02/SR4 2

\#F $019<$ fill area set $3>$ :

$02.03 .03 /$ SR0 $62.03 .03 /$ SR10 $02.03 .03 /$ SR14 $04.01 .06 /$ SR01 $04.01 .06 /$ SR07 $04.01 .06 /$ SR08 $04.03 .04 .01 /$ SR15 $05.02 /$ SR19 $05.02 /$ SR20 05.02/SR21 06.01.02/SR17 06.01.02/SR42

\#F $020<$ fill area set>:

$02.03 .03 /$ SR0 $62.03 .03 /$ SR10 $02.03 .03 /$ SR14 $04.01 .06 /$ SR0 4 $04.01 .06 /$ SR07 $04.01 .06 /$ SR08 $04.03 .04 .01 /$ SR15 $05.02 /$ SR19 
05.02/SR20 05.02/SR21 06.01.02/SR17 06.01.02/SR42

\#F $021<$ cell array 3>:

$02.03 .03 /$ SR06 02.03.03/SR10 $02.03 .03 /$ SR17 $04.01 .07 /$ SR01

$04.01 .07 /$ SR04 $04.01 .07 /$ SR05 $04.01 .07 /$ SR11 $04.01 .07 /$ SR12

$04.01 .07 /$ SR13 $04.01 .07 /$ SR14 $04.03 .04 .01 /$ SR15 $05.02 /$ SR19

$05.02 /$ SR20 05.02/SR21 $06.01 .02 /$ SR17 $06.01 .02 /$ SR4 2

\#F $022<$ cell array>:

02.03.03/SR06 02.03.03/SR10 02.03.03/SR17 04.01.07/SR06

$04.01 .07 /$ SR09 $04.01 .07 /$ SR10 $04.01 .07 /$ SR11 $04.01 .07 /$ SR12

$04.01 .07 /$ SR13 $04.01 .07 /$ SR14 $04.03 .04 .01 /$ SR15 $05.02 /$ SR19

$05.02 /$ SR20 $05.02 / S R 2106.01 .02 / S R 1706.01 .02 / S R 42$

\#F 023 <generalized drawing primitive 3>:

$02.03 .03 /$ SR0 $62.03 .03 /$ SR10 $04.01 .08 /$ SR01 $04.03 .04 .01 /$ SR15

$05.02 /$ SR19 $05.02 /$ SR20 $05.02 /$ SR21 $06.01 .02 / S R 1706.01 .02 / S R 42$

\#F 024 <generalized drawing primitive>:

$02.03 .03 /$ SR0 $62.03 .03 /$ SR10 $04.01 .08 /$ SR0 $604.03 .04 .01 /$ SR15

$05.02 /$ SR19 05.02/SR20 $05.02 / S R 2106.01 .02 / S R 1706.01 .02 / S R 42$

\#F 025 <set polyline index :

$04.02 .01 .02 /$ SR0 $34.02 .01 .02 /$ SR0 $404.02 .01 .02 /$ SR0 5

$04.02 .01 .02 /$ SR0 $64.02 .01 .02 /$ SR0 $04.02 .01 .02 /$ SR 35

$04.02 .01 .02 /$ SR4 $304.02 .01 .02 /$ SR4 $404.02 .01 .02 /$ SR 45

$04.02 .01 .02 /$ SR4 $705.02 /$ SR19 $05.02 /$ SR20 $05.02 /$ SR2 1

\#F 026 <set polymarker index>:

$04.02 .02 .02 /$ SR03 $04.02 .02 .02 /$ SR0 $404.02 .02 .02 /$ SR0 5

$04.02 .02 .02 /$ SR0 $64.02 .02 .02 /$ SR0 $704.02 .02 .02 /$ SR 35

$04.02 .02 .02 /$ SR $4304.02 .02 .02 /$ SR $44 \quad 04.02 .02 .02 / S R 45$

$04.02 .02 .02 /$ SR $4604.02 .02 .02 /$ SR4 $805.02 /$ SR19 $05.02 /$ SR2 0

$05.02 /$ SR2 1

\#F 027 <set text index>:

$04.02 .03 .02 /$ SR03 $04.02 .03 .02 /$ SR0 $404.02 .03 .02 /$ SR0 5

$04.02 .03 .02 /$ SR0 $64.02 .03 .02 /$ SR0 $04.02 .03 .02 /$ SR3 8

$04.02 .03 .02 /$ SR39 $04.02 .03 .02 /$ SR57 $04.02 .03 .02 /$ SR61

$04.02 .03 .02 /$ SR6 $404.02 .04 .02 /$ SR01 $04.02 .04 .02 /$ SR0 2

$04.02 .04 .02 /$ SR0 $304.02 .04 .02 /$ SR0 $404.02 .04 .02 /$ SR0 5

$04.02 .04 .02 /$ SR11 $04.02 .04 .02 /$ SR12 $04.02 .04 .02 /$ SR2 4

$04.02 .04 .02 /$ SR2 $704.02 .04 .02 /$ SR29 $05.02 /$ SR19 $05.02 /$ SR2 0

$05.02 / S R 21$

\#F 028 <set interior index>:

$04.02 .05 .02 /$ SR03 $04.02 .05 .02 /$ SR0 $404.02 .05 .02 /$ SR0 5

$04.02 .05 .02 /$ SR0 $04.02 .05 .02 /$ SR0 $04.02 .05 .02 /$ SR0 8

$04.02 .05 .02 /$ SR3 $04.02 .05 .02 /$ SR39 $04.02 .05 .02 /$ SR5 $105.02 /$ SR 19

$05.02 /$ SR2 $005.02 / S R 21$

\#F 029 <set edge index>:

$04.02 .05 .03 /$ SR03 $04.02 .05 .03 /$ SR0 $404.02 .05 .03 /$ SR0 5

$04.02 .05 .03 /$ SR0 $04.02 .05 .03 /$ SR0 $04.02 .05 .03 /$ SR3 0 
$04.02 .05 .03 /$ SR3 $804.02 .05 .03 /$ SR46 $04.02 .05 .03 /$ SR 47

$04.02 .05 .03 /$ SR $4804.02 .05 .03 /$ SR50 $05.02 /$ SR $1905.02 /$ SR2 0

$05.02 /$ SR2 1

\#F 030 <set linetype >

$04.02 .01 .01 /$ SR03 $04.02 .01 .01 /$ SR04 04.02.01.01/SR0 5

$04.02 .01 .01 /$ SR0 $64.02 .01 .01 /$ SR0 $05.02 /$ SR19 $05.02 /$ SR2 0

$05.02 /$ SR2 1

\#F 031 <set linewidth scale factors:

$04.02 .01 .01 /$ SR03 $04.02 .01 .01 /$ SR13 $04.02 .01 .01 /$ SR14

$04.02 .01 .01 /$ SR15 $04.02 .01 .01 /$ SR16 $05.02 /$ SR19 $05.02 /$ SR2 0

$05.02 /$ SR21

\#F 032 <set polyline colour index>:

$04.02 .01 .01 /$ SR03 $04.02 .01 .01 /$ SR19 $04.02 .01 .01 /$ SR2 0

$04.02 .01 .01 /$ SR21 $04.02 .01 .01 /$ SR2 $205.02 /$ SR19 $05.02 /$ SR2 0

$05.02 /$ SR2 1

\#F 033 <set marker types:

$04.02 .02 .01 /$ SR03 $04.02 .02 .01 /$ SR0 $404.02 .02 .01 /$ SR0 5

$04.02 .02 .01 /$ SR0 $64.02 .02 .01 /$ SR0 $0404.02 .02 .01 /$ SR $1705.02 /$ SR 19

$05.02 /$ SR20 $05.02 /$ SR21

\#F 034 <set marker size scale factors:

$04.02 .02 .01 /$ SR03 $04.02 .02 .01 /$ SR13 $04.02 .02 .01 /$ SR14

$04.02 .02 .01 /$ SR15 $04.02 .02 .01 /$ SR16 $04.02 .02 .01 /$ SR17 $05.02 /$ SR19

$05.02 / S R 2005.02 / S R 21$

\#F 035 <set polymarker colour index>:

$04.02 .02 .01 /$ SR03 $04.02 .02 .01 /$ SR2 $004.02 .02 .01 /$ SR2 1

$04.02 .02 .01 /$ SR2 $204.02 .02 .01 /$ SR23 $05.02 /$ SR19 $05.02 /$ SR2 0

$05.02 /$ SR2 1

\#F 036 <set text font>:

$04.02 .03 .01 /$ SR11 $04.02 .03 .01 /$ SR12 $04.02 .03 .01 /$ SR13

$04.02 .03 .01 /$ SR21 $04.02 .03 .01 /$ SR22 $04.02 .04 .01 /$ SR0 5

$04.02 .04 .01 /$ SR0 $64.02 .04 .01 /$ SR14 $04.02 .04 .01 /$ SR15 $05.02 /$ SR19

$05.02 / S R 20 \quad 05.02 / S R 21$

\#F 037 <set text precision>:

$04.02 .03 .01 /$ SR11 $04.02 .03 .01 /$ SR25 $04.02 .03 .01 /$ SR2 6

$04.02 .03 .01 /$ SR2 $704.02 .03 .01 /$ SR2 $04.02 .03 .01 /$ SR2 9

$04.02 .03 .01 /$ SR30 $04.02 .03 .01 /$ SR31 $04.02 .04 .01 /$ SR0 5

$04.02 .04 .01 /$ SR18 $04.02 .04 .01 /$ SR19 $04.02 .04 .01 /$ SR2 0

$04.02 .04 .01 /$ SR2 $104.02 .04 .01 /$ SR2 $204.02 .04 .01 /$ SR23 $05.02 /$ SR19

$05.02 / S R 20 \quad 05.02 / S R 21$

\#F 038 <set character expansion factor>:

$04.02 .03 .01 /$ SR11 $04.02 .03 .01 /$ SR34 $04.02 .03 .01 /$ SR3 5

$04.02 .03 .01 /$ SR3 $704.02 .04 .01 /$ SR0 $504.02 .04 .01 /$ SR2 6

$04.02 .04 .01 /$ SR29 $05.02 /$ SR19 $05.02 /$ SR20 $05.02 / S R 21$

\#F 039 <set character spacing>: 
$04.02 .03 .01 /$ SR11 $04.02 .03 .01 /$ SR40 $04.02 .03 .01 /$ SR 41 $04.02 .03 .01 /$ SR43 $04.02 .04 .01 /$ SR05 $04.02 .04 .01 /$ SR3 2 $04.02 .04 .01 /$ SR3 $405.02 /$ SR19 $05.02 /$ SR20 $05.02 /$ SR2 1

\#F 040 <set text colour index>:

$04.02 .03 .01 /$ SR11 $04.02 .03 .01 /$ SR46 $04.02 .03 .01 /$ SR 47 $04.02 .03 .01 /$ SR48 $04.02 .03 .01 /$ SR49 $04.02 .04 .01 /$ SR0 5 $04.02 .04 .01 /$ SR3 $704.02 .04 .01 /$ SR3 $804.02 .04 .01 /$ SR3 $0505.02 /$ SR 19 $05.02 /$ SR20 $05.02 /$ SR2 1

\#F 041 <set character height>:

$04.02 .03 .01 /$ SR03 $04.02 .03 .01 /$ SR11 $04.02 .03 .01 /$ SR52

$04.02 .03 .01 /$ SR5 $04.02 .03 .01 /$ SR61 $05.02 /$ SR $1905.02 /$ SR2 0 $05.02 /$ SR2 1

\#F 042 <set character up vectors:

$04.02 .03 .01 /$ SR11 $04.02 .03 .01 /$ SR79 $04.02 .03 .01 /$ SR80

$04.02 .03 .01 /$ SR81 $05.02 /$ SR19 $05.02 /$ SR20 $05.02 /$ SR2 1

\#F 043 <set text path>:

$04.02 .03 .01 /$ SR11 $04.02 .03 .01 /$ SR64 $04.02 .03 .01 /$ SR65

$04.02 .03 .01 /$ SR6 $64.02 .03 .01 /$ SR $6704.02 .03 .01 /$ SR 68

$04.02 .03 .01 /$ SR $6905.02 /$ SR19 $05.02 /$ SR20 $05.02 /$ SR2 1

\#F 044 <set text alignment>:

$04.02 .03 .01 /$ SR11 $04.02 .03 .01 /$ SR72 $04.02 .03 .01 /$ SR73

$04.02 .03 .01 /$ SR74 $04.02 .03 .01 /$ SR75 $04.02 .03 .01 /$ SR76 $05.02 /$ SR 19

$05.02 /$ SR2 $005.02 / S R 21$

\#F 045 <set annotation text character height>:

$04.02 .04 .01 /$ SR05 $04.02 .04 .01 /$ SR42 $04.02 .04 .01 /$ SR 48

$04.02 .04 .01 /$ SR51 $04.02 .04 .01 /$ SR52 $05.02 /$ SR $1905.02 /$ SR2 0

$05.02 /$ SR21

\#F 046 <set annotation text character up vectors:

$04.02 .04 .01 /$ SR05 $04.02 .04 .01 /$ SR70 $04.02 .04 .01 /$ SR 71

$04.02 .04 .01 /$ SR72 $05.02 /$ SR19 $05.02 /$ SR2 $0 \quad 05.02 /$ SR2 1

\#F 047 <set annotation text path>:

$04.02 .04 .01 /$ SR05 $04.02 .04 .01 /$ SR55 $04.02 .04 .01 /$ SR5 6

$04.02 .04 .01 /$ SR5 $704.02 .04 .01 /$ SR58 $04.02 .04 .01 /$ SR5 9

$04.02 .04 .01 /$ SR60 $05.02 /$ SR19 $05.02 /$ SR20 $05.02 /$ SR2 1

\#F 048 <set annotation text alignment>:

$04.02 .04 .01 /$ SR05 $04.02 .04 .01 /$ SR63 04.02.04.01/SR64

$04.02 .04 .01 /$ SR65 $04.02 .04 .01 /$ SR $66 \quad 04.02 .04 .01 /$ SR $6705.02 /$ SR 19

$05.02 / S R 20 \quad 05.02 / S R 21$

\#F 049 <set annotation style>:

$04.02 .04 .01 /$ SR05 $04.02 .04 .01 /$ SR75 $04.02 .04 .01 /$ SR76

$04.02 .04 .01 /$ SR78 $04.02 .04 .01 /$ SR79 $04.02 .04 .01 /$ SR80

$04.02 .04 .01 /$ SR81 $04.02 .04 .01 /$ SR82 $05.02 /$ SR19 $05.02 /$ SR20

$05.02 /$ SR21 
\#F 050 <set interior styles:

$04.02 .05 .01 /$ SR03 $04.02 .05 .01 /$ SR0 $404.02 .05 .01 /$ SR0 5

$04.02 .05 .01 /$ SR0 $64.02 .05 .01 /$ SR0 $704.02 .05 .01 /$ SR0 8

$04.02 .05 .01 /$ SR09 $04.02 .05 .01 /$ SR10 $04.02 .05 .01 /$ SR12

$04.02 .05 .01 /$ SR13 $04.02 .05 .01 /$ SR14 $04.02 .05 .01 /$ SR15 $05.02 /$ SRI9

$05.02 /$ SR2 $005.02 / S R 21$

\#F 051 <set interior style index s:

$04.02 .05 .01 /$ SR03 $04.02 .05 .01 /$ SR0 $404.02 .05 .01 /$ SR14

$04.02 .05 .01 /$ SR15 $04.02 .05 .01 /$ SR18 $04.02 .05 .01 /$ SR19

$04.02 .05 .01 /$ SR20 $04.02 .05 .01 /$ SR21 05.02/SR19 $05.02 /$ SR20

$05.02 /$ SR2 1

\#F 052 <set interior colour index>:

$04.02 .05 .01 /$ SR03 $04.02 .05 .01 /$ SR0 $404.02 .05 .01 /$ SR10

$04.02 .05 .01 /$ SR13 $04.02 .05 .01 /$ SR14 $04.02 .05 .01 /$ SR2 6

$04.02 .05 .01 /$ SR2 $704.02 .05 .01 /$ SR2 $804.02 .05 .01 /$ SR2 $905.02 /$ SR 19

$05.02 /$ SR20 $05.02 /$ SR2 1

\#F $053<$ set edge flag>:

$04.02 .05 .01 /$ SR0 $404.02 .05 .01 /$ SR32 $04.02 .05 .01 /$ SR3 3

$04.02 .05 .01 /$ SR3 $404.02 .05 .01 /$ SR3 $504.02 .05 .01 /$ SR3 $05.02 /$ SR 19

$05.02 / \mathrm{SR} 2005.02 / \mathrm{SR} 21$

\#F 054 <set edgetype>:

$04.02 .05 .01 /$ SR0 $404.02 .05 .01 /$ SR40 $04.02 .05 .01 / S R 41$

$04.02 .05 .01 /$ SR42 $04.02 .05 .01 /$ SR43 $05.02 /$ SR19 $05.02 /$ SR20

$05.02 /$ SR21

\#F 055 <set edgewidth scale factors:

$04.02 .05 .01 /$ SR0 $404.02 .05 .01 /$ SR49 $04.02 .05 .01 /$ SR 50

$04.02 .05 .01 /$ SR51 $04.02 .05 .01 /$ SR52 $05.02 /$ SR19 $05.02 /$ SR20

$05.02 /$ SR 21

\#F 056 <set edge colour index>:

$04.02 .05 .01 /$ SR0 $404.02 .05 .01 /$ SR55 $04.02 .05 .01 /$ SR5 6

$04.02 .05 .01 /$ SR5 $704.02 .05 .01 /$ SR5 $805.02 /$ SR $1905.02 /$ SR 20

$05.02 / S R 21$

\#F 057 <set pattern size>:

$04.02 .05 .01 /$ SR03 $04.02 .05 .01 /$ SR04 04.02.05.01/SR61

$04.02 .05 .01 /$ SR62 $04.02 .05 .01 /$ SR63 $04.02 .05 .01 /$ SR 71

$04.02 .05 .01 /$ SR73 $04.02 .05 .01 /$ SR74 $04.02 .05 .01 /$ SR76

$04.02 .05 .01 /$ SR77 $05.02 /$ SR19 $05.02 /$ SR20 $05.02 / S R 21$

\#F 058 <set pattern reference point and vectors >

$04.02 .05 .01 /$ SR0 3 04.02.05.01/SR04 04.02.05.01/SR6 6

$04.02 .05 .01 /$ SR68 $04.02 .05 .01 /$ SR70 $04.02 .05 .01 /$ SR71

$04.02 .05 .01 /$ SR72 $04.02 .05 .01 /$ SR73 $04.02 .05 .01 /$ SR74

$04.02 .05 .01 /$ SR76 $04.02 .05 .01 /$ SR7 $705.02 /$ SR $1905.02 /$ SR20

$05.02 /$ SR 21

\#F 059 <set pattern reference point>:

$04.02 .05 .01 /$ SR03 $04.02 .05 .01 /$ SR0 $404.02 .05 .01 /$ SR6 7 
$04.02 .05 .01 /$ SR69 $04.02 .05 .01 /$ SR71 $04.02 .05 .01 /$ SR72

$04.02 .05 .01 /$ SR73 $04.02 .05 .01 /$ SR74 $04.02 .05 .01 /$ SR76

$04.02 .05 .01 /$ SR77 $05.02 /$ SR19 $05.02 /$ SR20 $05.02 /$ SR2 1

\#F $060<$ add names to set>:

$02.03 .03 /$ SR0 $602.03 .03 /$ SR18 $02.03 .03 /$ SR19 $02.03 .03 /$ SR20

$04.03 .04 .01 /$ SR01 $04.03 .04 .01 /$ SR09 $04.03 .04 .01 /$ SR10

$04.03 .04 .01 /$ SR12 $04.03 .04 .01 /$ SR15 $04.03 .04 .02 /$ SR0 4

$04.03 .04 .02 /$ SR0 $84.03 .04 .02 /$ SR09 $05.02 /$ SR19 $05.02 /$ SR2 0

$05.02 /$ SR2 1

\#F 061 <remove names from set>:

$02.03 .03 /$ SR0 $602.03 .03 /$ SR18 $02.03 .03 /$ SR19 $02.03 .03 /$ SR2 0

$04.03 .04 .01 /$ SR0 $404.03 .04 .01 /$ SR09 $04.03 .04 .01 /$ SR 10

$04.03 .04 .01 /$ SR13 $04.03 .04 .01 /$ SR15 $04.03 .04 .02 /$ SR0 4

$04.03 .04 .02 /$ SR08 $04.03 .04 .02 /$ SR09 $05.02 /$ SR19 $05.02 /$ SR2 0

$05.02 /$ SR2 1

\#F 062 <set individual asf $>$ :

$04.02 .01 .02 /$ SR35 $04.02 .01 .02 /$ SR43 $04.02 .01 .02 / S R 44$

$04.02 .01 .02 /$ SR4 $504.02 .01 .02 /$ SR4 $704.02 .02 .02 /$ SR 35

$04.02 .02 .02 /$ SR $4304.02 .02 .02 /$ SR $44 \quad 04.02 .02 .02 / S R 45$

$04.02 .02 .02 /$ SR4 $604.02 .02 .02 /$ SR4 $804.02 .03 .02 /$ SR3 8

$04.02 .03 .02 /$ SR39 $04.02 .03 .02 /$ SR57 $04.02 .03 .02 /$ SR61

$04.02 .03 .02 /$ SR64 $04.02 .04 .02 /$ SR11 $04.02 .04 .02 /$ SR12

$04.02 .04 .02 /$ SR2 $404.02 .04 .02 /$ SR2 $704.02 .04 .02 /$ SR2 9

$04.02 .05 .02 /$ SR35 $04.02 .05 .02 /$ SR39 $04.02 .05 .02 /$ SR5 1

$04.02 .05 .03 /$ SR30 $04.02 .05 .03 /$ SR3 $804.02 .05 .03 /$ SR4 6

$04.02 .05 .03 / S R 4704.02 .05 .03 / S R 48 \quad 04.02 .05 .03 / S R 50 \quad 04.03 .01 / S R 01$

$04.03 .01 /$ SR04 $04.03 .01 /$ SR0 $504.03 .01 /$ SR0 $604.03 .01 /$ SR0 7

$04.03 .01 /$ SR08 $04.03 .01 /$ SR09 $05.02 /$ SR19 $05.02 /$ SR2 $0 \quad 05.02 /$ SR2 1

\#F 063 <set polyline representation>:

$04.02 .01 .02 /$ SR10 $04.02 .01 .02 /$ SR11 $04.02 .01 .02 /$ SR18

$04.02 .01 .02 /$ SR3 $304.02 .01 .02 /$ SR34 $04.02 .01 .02 /$ SR3 5

$04.02 .01 .02 /$ SR4 $104.02 .01 .02 /$ SR42 $04.02 .01 .02 /$ SR 43

$04.02 .01 .02 /$ SR $4404.02 .01 .02 /$ SR $45 \quad 04.02 .01 .02 /$ SR 46

$04.02 .01 .02 /$ SR $4705.02 /$ SR19 05.02/SR20 $05.02 /$ SR2 1

\#F 064 <set polymarker representation>:

$04.02 .02 .02 /$ SR10 $04.02 .02 .02 /$ SR11 $04.02 .02 .02 /$ SR18

$04.02 .02 .02 /$ SR3 $34.02 .02 .02 /$ SR3 $404.02 .02 .02 /$ SR3 5

$04.02 .02 .02 /$ SR4 $104.02 .02 .02 /$ SR4 $204.02 .02 .02 /$ SR 43

$04.02 .02 .02 /$ SR $4404.02 .02 .02 /$ SR4 $504.02 .02 .02 / S R 46$

$04.02 .02 .02 /$ SR $4704.02 .02 .02 /$ SR $48 \quad 05.02 /$ SR $1905.02 /$ SR2 0

$05.02 /$ SR2 1

\#F 065 <set text representation>:

$04.02 .03 .02 /$ SR10 $04.02 .03 .02 /$ SR11

$04.02 .03 .02 /$ SR $3204.02 .03 .02 /$ SR33

$04.02 .03 .02 /$ SR3 $54.02 .03 .02 /$ SR3 6

$04.02 .03 .02 /$ SR3 9

$04.02 .03 .02 /$ SR 40

$04.02 .03 .02 /$ SR18

$04.02 .03 .02 /$ SR3 4

$04.02 .03 .02 /$ SR 38

$04.02 .03 .02 /$ SR 53

$04.02 .03 .02 /$ SR5 5

$04.02 .03 .02 /$ SR 47

$04.02 .03 .02 /$ SR 57

$04.02 .03 .02 /$ SR 60

$04.02 .03 .02 /$ SR 56

$04.02 .03 .02 /$ SR61 
$04.02 .03 .02 /$ SR63 $04.02 .03 .02 /$ SR64 $04.02 .04 .02 /$ SR 10 $04.02 .04 .02 /$ SR11 $04.02 .04 .02 /$ SR $1204.02 .04 .02 /$ SRI3 $04.02 .04 .02 /$ SR20 $04.02 .04 .02 /$ SR2 $4 \quad 04.02 .04 .02 / S R 27$ $04.02 .04 .02 /$ SR29 $05.02 /$ SR19 $05.02 /$ SR20 $05.02 / S R 21$

\#F 066 <set interior representation>:

$04.02 .05 .02 /$ SR11 $04.02 .05 .02 /$ SR12 $04.02 .05 .02 /$ SR19 $04.02 .05 .02 /$ SR3 $104.02 .05 .02 /$ SR33 $04.02 .05 .02 /$ SR3 5 $04.02 .05 .02 /$ SR3 $704.02 .05 .02 /$ SR3 $804.02 .05 .02 /$ SR 39 $04.02 .05 .02 /$ SR40 $04.02 .05 .02 / S R 4104.02 .05 .02 / S R 50$ $04.02 .05 .02 /$ SR51 $05.02 /$ SR19 $05.02 /$ SR2 $005.02 / S R 21$

\#F 067 <set edge representations:

$04.02 .05 .03 /$ SR10 $04.02 .05 .03 /$ SR11 $04.02 .05 .03 /$ SR18 $04.02 .05 .03 /$ SR29 $04.02 .05 .03 /$ SR30 $04.02 .05 .03 /$ SR 36 $04.02 .05 .03 /$ SR $3704.02 .05 .03 /$ SR $3804.02 .05 .03 /$ SR 44 $04.02 .05 .03 /$ SR4 $504.02 .05 .03 /$ SR4 $604.02 .05 .03 / S R 47$ $04.02 .05 .03 /$ SR48 $04.02 .05 .03 /$ SR49 $04.02 .05 .03 /$ SR50 $05.02 /$ SR 19 $05.02 /$ SR20 $05.02 /$ SR2 1

\#F 068 <set pattern representation>:

$04.02 .05 .01 /$ SR75 $04.02 .05 .01 /$ SR76 $04.02 .05 .01 /$ SR7 7

$04.02 .05 .04 /$ SR01 $04.02 .05 .04 /$ SR02 $04.02 .05 .04 /$ SR10 $04.02 .05 .04 /$ SR19 $04.02 .05 .04 /$ SR20 $04.02 .05 .04 /$ SR21 $05.02 /$ SR19 $05.02 /$ SR20 $05.02 / S R 21$

\#F 069 <set colour representation>:

$04.03 .02 .01 /$ SR01 $04.03 .02 .01 /$ SR02 $04.03 .02 .01 /$ SR0 8

$04.03 .02 .02 /$ SR03 $04.03 .02 .02 /$ SR0 $504.03 .02 .02 /$ SR0 6

$04.03 .02 .02 /$ SR21 $04.03 .02 .02 /$ SR2 $604.03 .02 .02 /$ SR2 7

$04.03 .02 .02 /$ SR $3004.03 .02 .02 /$ SR $3104.03 .02 .02 /$ SR3 $305.02 /$ SR 19 $05.02 / S R 2005.02 / S R 21$

\#F 070 <set highlighting filter>: $04.03 .04 .02 /$ SR01 $04.03 .04 .02 /$ SR0 $404.03 .04 .02 /$ SR 10

$04.03 .04 .02 /$ SR11 $04.03 .04 .02 /$ SR12 $05.02 /$ SR19 $05.02 /$ SR20 $05.02 /$ SR2 1

\#F 071 <set invisibility filter>: $04.03 .04 .02 /$ SR05 $04.03 .04 .02 /$ SR0 $04.03 .04 .02 /$ SR09 $04.03 .04 .02 /$ SR10 $04.03 .04 .02 /$ SR11 $04.03 .04 .02 /$ SR12 $05.02 /$ SR19 $05.02 /$ SR2 $005.02 / S R 21$

\#F 072 <set colour model > :

$04.03 .02 .01 /$ SR0 $504.03 .02 .01 /$ SR09 $04.03 .02 .02 /$ SR17

$04.03 .02 .02 /$ SR2 $104.03 .02 .02 /$ SR 32

\#F 073 <set hlhsr identifier>:

$04.03 .03 /$ SR01 05.02/SR19 05.02/SR20 05.02/SR21

\#F 074 <set hlhsr mode> :

$04.03 .03 /$ SR0 $404.03 .03 /$ SR0 $604.03 .03 /$ SR0 $04.03 .03 /$ SRII

$05.02 /$ SR $1.905 .02 /$ SR20 $05.02 /$ SR2 1 
\#F 075 <set local transformation $3>$ :

$02.03 .03 /$ SR10 $05.02 /$ SR19 $05.02 /$ SR20 $05.02 /$ SR21 $06.01 .02 /$ SR01

$06.01 .02 /$ SR07 06.01.02/SR08 $06.01 .02 /$ SR16 $06.01 .02 /$ SR17

\#F 076 <set local transformation>:

$02.03 .03 /$ SR10 $05.02 /$ SR19 $05.02 /$ SR20 $05.02 /$ SR21 $06.01 .02 /$ SR0 4

06.01.02/SR07 06.01.02/SR16 06.01.02/SR17 $06.01 .02 /$ SR18

\#F 077 <set global transformation 3>:

$02.03 .03 /$ SR10 $05.02 /$ SR19 $05.02 /$ SR20 $05.02 /$ SR21 $06.01 .02 /$ SR0 9 $06.01 .02 /$ SR15 $06.01 .02 /$ SR16 $06.01 .02 /$ SR 17

\#F 078 <set global transformation>:

$02.03 .03 /$ SR10 $05.02 /$ SR19 $05.02 /$ SR20 $05.02 /$ SR2 $106.01 .02 /$ SR12 $06.01 .02 /$ SR16 $06.01 .02 /$ SR17 $06.01 .02 /$ SR18

\#F 079 <set modelling clipping volume $3>$ :

$02.03 .03 /$ SR10 $05.02 /$ SR19 $05.02 /$ SR20 $05.02 / S R 2106.01 .02 / S R 24$

$06.01 .02 /$ SR38 $06.01 .02 /$ SR39 $06.01 .02 /$ SR40 $06.01 .02 /$ SR 41

$06.01 .02 / S R 4206.01 .02 / S R 4506.01 .02 / S R 4606.01 .02 / S R 47$

$06.01 .02 /$ SR 48

\#F 080 <set modelling clipping volumes:

$02.03 .03 /$ SR10 $05.02 /$ SR19 $05.02 /$ SR20 $05.02 /$ SR21 $06.01 .02 /$ SR19

$06.01 .02 /$ SR27 06.01.02/SR38 06.01.02/SR39 $06.01 .02 /$ SR 40

$06.01 .02 /$ SR41 $06.01 .02 /$ SR 42

\#F 081 <set modelling clipping indicator>:

05.02/SR19 05.02/SR20 05.02/SR21 06.01.02/SR30 06.01.02/SR36

\#F 082 <restore modelling clipping volume>:

$02.03 .03 /$ SR10 05.02/SR19 05.02/SR20 05.02/SR21 06.01.02/SR33

$06.01 .02 / S R 4206.01 .02 / S R 49$

\#F 083 <set view index>:

$05.02 /$ SR10 $05.02 /$ SR19 $05.02 /$ SR20 $05.02 /$ SR21 $06.02 .02 /$ SR01

\#F 084 <set view representation $3>$ :

05.02/SR10 05.02/SR19 $05.02 /$ SR20 05.02/SR21 $06.02 .02 /$ SR14

$06.02 .02 /$ SR $1506.02 .02 /$ SR23

\#F 085 <set view representation>:

$05.02 /$ SR10 $05.02 /$ SR19 $05.02 /$ SR20 $05.02 /$ SR21 $06.02 .02 /$ SR14

$06.02 .02 /$ SR15 $06.02 .02 /$ SR16 $06.02 .02 /$ SR2 3

\#F 086 <set view transformation input priority>:

$06.02 .02 / S R 2106.02 .02 / S R 22$

\#F 087 <set workstation window $3>$ :

$05.02 /$ SR10 05.02/SR19 $05.02 /$ SR20 $05.02 /$ SR21 $06.03 /$ SR0 $606.03 /$ SR0 8 $06.03 /$ SR14

\#F 088 <set workstation window>:

05.02/SR10 05.02/SR19 05.02/SR20 05.02/SR21 06.03/SR07 06.03/SR08 
FUNCTION CROSS-REFERENCE

$06.03 /$ SR14

\#F 089 <set workstation viewport $3>$ :

$05.02 /$ SR10 $05.02 /$ SR19 $05.02 /$ SR20 $05.02 /$ SR21 $06.03 /$ SR09 $06.03 /$ SR11

$06.03 /$ SR14

\#F 090 <set workstation viewport>:

05.02/SR10 05.02/SR19 05.02/SR20 05.02/SR21 06.03/SR10 06.03/SR11

$06.03 /$ SR14

\#F 091 <translate 3>:

06.01.01/SR01 06.01.01/SR02 06.01.01/SR03

\#F 092 <translate>:

06.01.01/SR01 06.01.01/SR02 06.01.01/SR04

\#F $093<$ scale 3>:

06.01.01/SR01 06.01.01/SR02 06.01.01/SR05

\#F 094 <scale>:

06.01.01/SR01 06.01.01/SR02 06.01.01/SR06

\#F 095 <rotate $\mathrm{x}>$ :

06.01.01/SR01 06.01.01/SR02 06.01.01/SR07

\#F 096 <rotate $y>$ :

06.01.01/SR01 06.01.01/SR02 06.01.01/SR08

\#F 097 <rotate $z>$ :

06.01.01/SR01 06.01.01/SR02 06.01.01/SR09

\#F 098 <rotate>:

06.01.01/SR01 06.01.01/SR02 06.01.01/SR10

\#F $099<$ compose matrix 3>:

06.01.01/SR01 06.01.01/SR02 06.01.01/SR11

\#F 100 <compose matrix>:

06.01.01/SR01 06.01.01/SR02 06.01.01/SR12

\#F 101 <transform point 3>:

$06.01 .01 /$ SR01 06.01.01/SR02 06.01.01/SR13

\#F 102 <transform point>:

06.01.01/SR01 06.01.01/SR02 06.01.01/SR14

\#F 103 <build transformation matrix 3>:

06.01.01/SR01 06.01.01/SR02 06.01.01/SR15

\#F 104 <build transformation matrix>:

06.01.01/SR01 06.01.01/SR02 06.01.01/SR16

\#F 105 <compose transformation matrix 3>:

06.01.01/SR01 06.01.01/SR02 06.01.01/SR17 
\#F 106 <compose transformation matrix>:

06.01.01/SR01 06.01.01/SR02 06.01.01/SR18

\#F 107 <evaluate view orientation matrix 3>:

$06.02 .01 /$ SR01 $06.02 .01 /$ SR02 $06.02 .01 /$ SR03 $06.02 .01 /$ SR0 4

\#F 108 <evaluate view orientation matrix>:

06.02.01/SR01 06.02.01/SR02 06.02.01/SR05 $06.02 .01 /$ SR06

\#F 109 <evaluate view mapping matrix 3>:

$06.02 .01 /$ SR01 06.02.01/SR02 06.02.01/SR07 $06.02 .01 /$ SR0 8

$06.02 .01 /$ SR09 $06.02 .01 /$ SR10

\#F 110 <evaluate view mapping matrix>:

$06.02 .01 /$ SR01 $06.02 .01 /$ SR02 $06.02 .01 /$ SR11 $06.02 .01 /$ SR12

$06.02 .01 /$ SR13

\#F 111 <open structure>:

$02.01 .01 /$ SR01 02.01.01/SR09 $02.01 .01 /$ SR10 $02.02 .01 /$ SR01

$02.02 .01 /$ SR02 $02.02 .01 /$ SR03 $05.03 /$ SR09

\#F $112<$ close structure>:

$02.02 .01 / \mathrm{SR} 04$

\#F 113 <execute structure>:

$02.01 /$ SR01 02.01/SR05 02.01/SR06 02.01.01/SR03 02.01.01/SR09

$02.01 .01 /$ SR $1002.03 .03 /$ SR02 $03.04 /$ SR0 $0303.04 /$ SR0 $803.04 /$ SR09

$04.02 .01 .01 /$ SR12 $04.02 .01 .01 /$ SR1.8 $04.02 .01 .01 /$ SR2 4

$04.02 .01 .02 /$ SR09 $04.02 .02 .01 /$ SR12. $04.02 .02 .01 /$ SR19

$04.02 .02 .01 /$ SR25 $04.02 .02 .02 /$ SR09 $04.02 .03 .01 /$ SR2 4

$04.02 .03 .01 /$ SR33 $04.02 .03 .01 /$ SR39 $04.02 .03 .01 /$ SR 45

$04.02 .03 .01 /$ SR51 $04.02 .03 .01 /$ SR63 $04.02 .03 .01 /$ SR71

$04.02 .03 .01 /$ SR78 $04.02 .03 .01 /$ SR83 $04.02 .03 .02 /$ SR0 9

$04.02 .04 .01 /$ SR17 $04.02 .04 .01 /$ SR2 $504.02 .04 .01 /$ SR 31

$04.02 .04 .01 /$ SR36 $04.02 .04 .01 /$ SR $4104.02 .04 .01 /$ SR 54

$04.02 .04 .01 /$ SR62 $04.02 .04 .01 /$ SR69 $04.02 .04 .01 /$ SR74

$04.02 .04 .01 /$ SR8 $404.02 .04 .02 /$ SR0 $0404.02 .05 .01 /$ SR 17

$04.02 .05 .01 /$ SR25 $04.02 .05 .01 /$ SR31 $04.02 .05 .01 /$ SR3 9

$04.02 .05 .01 /$ SR48 $04.02 .05 .01 /$ SR54 $04.02 .05 .01 /$ SR 60

$04.02 .05 .01 /$ SR65 $04.02 .05 .01 /$ SR81 $04.02 .05 .01 /$ SR83

$04.02 .05 .02 /$ SR10 $04.02 .05 .03 /$ SR09 $04.03 .01 /$ SR $1104.03 .04 .01 /$ SR 14

$05.02 /$ SR19 05.02/SR20 $05.02 /$ SR21 $05.03 /$ SR09 $06.01 .02 /$ SR2 1

$06.01 .02 /$ SR22 $06.01 .02 /$ SR23 $06.01 .02 /$ SR 44

\#F $114<1$ abel>:

$02.02 .02 /$ SR01

\#F 115 <application data>:

$02.02 .02 / \mathrm{SR} 04$

\#F 116 <generalized structure element>:

$02.02 .02 /$ SR07 02.02.02/SR08 $02.02 .02 /$ SR11 $05.02 /$ SR19 05.02/SR20

$05.02 /$ SR2 1 


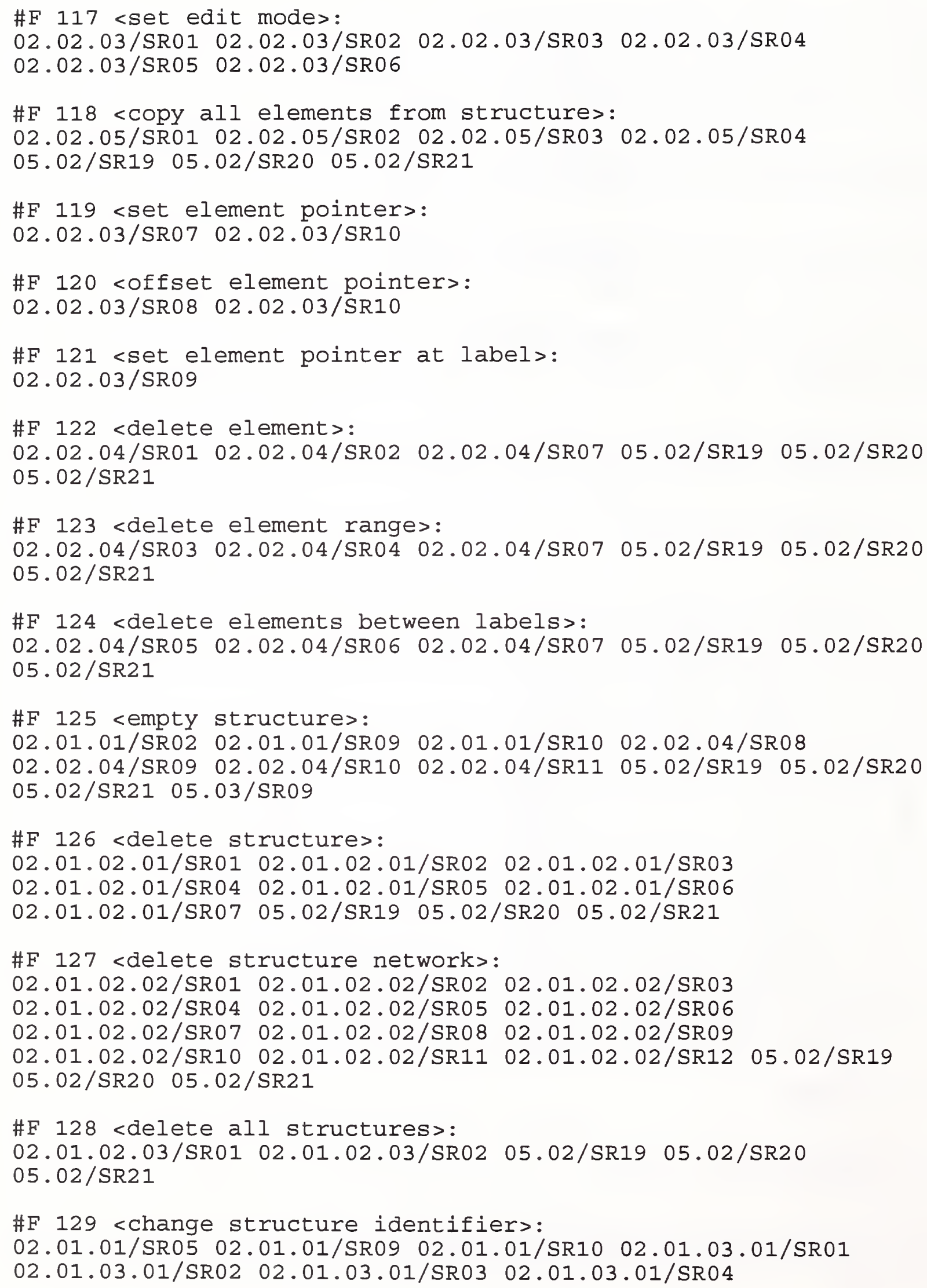

\#F 129 <change structure identifier>:

02.01.01/SR05 02.01.01/SR09 02.01.01/SR10 02.01.03.01/SR01 $02.01 .03 .01 /$ SR02 02.01.03.01/SR03 02.01.03.01/SR04 
$02.01 .03 .01 /$ SR05 $02.01 .03 .01 /$ SR06 $05.02 /$ SR19 $05.02 /$ SR20

$05.02 /$ SR2 1

\#F 130 <change structure references>:

$02.01 .01 /$ SR06 02.01.01/SR09 02.01.01/SR10 02.01.03.02/SR01

$02.01 .03 .02 /$ SR02 $02.01 .03 .02 /$ SR03 $02.01 .03 .02 /$ SR0 4

$02.01 .03 .02 /$ SR05 $02.01 .03 .02 /$ SR0 $62.01 .03 .03 /$ SR0 7

$02.01 .03 .03 /$ SR08 $05.02 /$ SR19 $05.02 /$ SR20 $05.02 /$ SR2 1

\#F 131 <change structure identifier and references>:

$02.01 .01 /$ SR07 02.01.01/SR09 02.01.01/SR10 02.01.03.03/SR01

$02.01 .03 .03 /$ SR02 $02.01 .03 .03 /$ SR03 $02.01 .03 .03 /$ SR0 4

$02.01 .03 .03 /$ SR05 $02.01 .03 .03 /$ SR0 $62.01 .03 .03 /$ SR0 7

$02.01 .03 .03 /$ SR0 $85.02 /$ SR19 $05.02 /$ SR20 $05.02 /$ SR2 1

\#F 132 <post structure>:

02.01.01/SR04 02.01.01/SR09 $02.01 .01 /$ SR10 $05.02 /$ SR10 05.02/SR19

$05.02 / S R 2005.02 / S R 2105.03 /$ SR01 $05.03 /$ SR02 $05.03 /$ SR03

$06.01 .02 /$ SR20 $06.01 .02 /$ SR 43

\#F 133 <unpost structure>:

$05.02 /$ SR19 05.02/SR20 05.02/SR21 05.03/SR04 05.03/SR05 05.03/SR06

\#F 134 <unpost all structures >:

$05.02 /$ SR19 05.02/SR20 05.02/SR21 05.03/SR07 05.03/SR0 8

\#F 135 <open archive files:

$03.01 /$ SR0 $503.01 /$ SR06 $03.01 /$ SR0 7

\#F 136 <close archive files:

$03.01 /$ SR08 $03.01 /$ SR09

\#F 137 <archive structures>:

$03.01 /$ SR0 $703.03 /$ SR01 03.03/SR04 03.03/SR05 03.03/SR06 03.03/SR07 $03.03 /$ SR0 8

\#F 138 <archive structure networks > :

$03.01 /$ SR0 $703.03 /$ SR02 $03.03 /$ SR0 $403.03 /$ SR0 $503.03 /$ SR0 $03.03 /$ SR0 03 $03.03 /$ SRO 8

\#F 139 <archive all structures>:

03.01 /SR0 $703.03 /$ SR03 03.03/SR0 $403.03 /$ SR0 $503.03 /$ SR0 $03.03 /$ SR0 7 $03.03 /$ SR0 8

\#F $140<$ set conflict resolution>:

$03.02 /$ SR02

\#F 141 <retrieve structure identifiers>:

$03.01 /$ SR0 $703.04 /$ SR01

\#F 142 <retrieve structures>:

03.01 /SR07 03.03/SR09 $03.03 /$ SR12 $03.03 /$ SR13 $03.03 /$ SR14 03.03/SR15

$03.03 /$ SR16 $03.03 /$ SR17 $03.03 /$ SR18 $03.03 /$ SR19 $05.02 /$ SR19 $05.02 /$ SR20

$05.02 /$ SR2 1 
\#F 143 <retrieve structure networks>:

$03.01 /$ SR07 03.03/SR10 03.03/SR12 $03.03 /$ SR13 $03.03 /$ SR14 03.03/SR15

$03.03 /$ SR16 03.03/SR17 $03.03 /$ SR18 $03.03 /$ SR19 $05.02 /$ SR19 $05.02 /$ SR20 $05.02 / S R 21$

\#F 144 <retrieve all structures>:

$03.01 /$ SR0 $703.03 /$ SR11 $03.03 /$ SR12 $03.03 /$ SR13 $03.03 /$ SR14 03.03/SR15 $03.03 /$ SR16 03.03/SR17 03.03/SR18 $03.03 /$ SR19 $05.02 /$ SR19 $05.02 /$ SR2 0 $05.02 /$ SR21

\#F 145 <retrieve paths to ancestors>:

$03.01 /$ SR0 $703.04 /$ SR0 $503.04 /$ SR0 $703.04 /$ SR0 $803.04 /$ SR10 $03.04 /$ SR 12 $03.04 /$ SR13 $03.04 /$ SR16

\#F 146 <retrieve paths to descendants >:

$03.01 /$ SR0 $703.04 /$ SR0 $63.04 /$ SR0 $0303.04 /$ SR0 $03.04 /$ SR09 $03.04 /$ SR 11 $03.04 /$ SR14 $03.04 /$ SR15 $03.04 /$ SR17

\#F 147 <delete structures from archives:

03.01 /SR0 $703.05 /$ SR01 03.05/SR04 03.05/SR05

\#F 148 <delete structure networks from archive>:

$03.01 /$ SR0 $703.05 /$ SR02 03.05/SR0 $403.05 /$ SR0 5

\#F 149 <delete all structures from archive>:

$03.01 /$ SR0 $703.05 /$ SR03 03.05/SR04 03.05/SR05

\#F 150 <set pick identifier>:

05.02/SR19 05.02/SR20 05.02/SR21

\#F 164 <set locator modes:

$05.02 /$ SR30

\#F 165 <set stroke modes:

$05.02 /$ SR 30

\#F 166 <set valuator modes:

$05.02 /$ SR 30

\#F 167 <set choice modes:

$05.02 /$ SR 30

\#F 168 <set pick mode> :

$05.02 /$ SR 30

\#F 169 <set string modes:

$05.02 /$ SR3 0

\#F 170 <request locator $3>$ :

$05.02 /$ SR 30

\#F 171 <request locator>:

$05.02 /$ SR 30 
\#F 172 <request stroke 3>:

$05.02 / \operatorname{SR} 30$

\#F 173 <request stroke> :

$05.02 /$ SR 30

\#F 174 <request valuator>:

$05.02 /$ SR 30

\#F 175 <request choice>:

$05.02 / \operatorname{SR} 30$

\#F 176 <request pick>:

$05.02 /$ SR 30

\#F 177 <request string>:

$05.02 /$ SR 30

\#F 200 <inquire system state value>:

$01 /$ SRO 3

\#F 201 <inquire workstation state value>:

05.01.01/SR02 05.01.01/SR03 05.01.01/SR04 05.01.01/SR05

\#F 202 <inquire structure state values:

$02.02 .01 /$ SR09

\#F 203 <inquire archive state values:

$03.01 /$ SR03 03.01/SR05 03.01/SR08 03.01/SR10

\#F 204 <inquire list of available workstation types>:

05.01.02/SR04 05.01.02/SR05 05.01.02/SR06 05.01.02/SR07

\#F 205 <inquire phigs facilities>:

$02.03 .03 /$ SR21 02.03.03/SR22 03.01/SR01 03.01/SR02 04.01.03/SR07

$04.01 .03 /$ SR08 $04.03 .04 .01 /$ SR0 $04.03 .04 .01 /$ SR08 $05.01 .01 /$ SR09

$05.01 .01 /$ SR10 $05.01 .01 /$ SR11

\#F 206 <inquire generalized structure element facilities>:

$02.02 .02 /$ SR11 $02.02 .02 /$ SR12

\#F 207 <inquire modelling clipping facilities>:

$06.01 .02 /$ SR37 $06.01 .02 /$ SR38 $06.01 .02 /$ SR 39

\#F 208 <inquire edit mode>:

$02.02 .03 /$ SR 11

\#F 209 <inquire set of open workstations>:

05.01.01/SR01 05.01.01/SR06 05.01.01/SR07 05.01.01/SR12

\#F 210 <inquire structure identifiers >:

02.01/SR02 02.01.01/SR09 
FUNCTION CROSS-REFERENCE

\#F 211 <inquire archive files>:

03.01 /SR04 03.01/SR06 03.01/SR09 03.01/SR11

\#F 212 <inquire conflict resolution>:

$03.02 /$ SR01 $03.02 /$ SR03

\#F 213 <inquire all conflicting structures>:

$03.04 /$ SRO2

\#F 214 <inquire conflicting structures in networks:

$03.04 /$ SR03 $03.04 /$ SR0 4

\#F 216 <inquire workstation connection and types:

$05.01 .02 /$ SR08

\#F 217 <inquire list of view indices>:

06.02 .02 /SR08 06.02.02/SR18 $06.02 .02 /$ SR19 $06.02 .02 /$ SR20

\#F 218 <inquire view representation>:

06.02.02/SR09 06.02.02/SR10 $06.02 .02 /$ SRI1 $06.02 .02 /$ SR17

$06.02 .02 /$ SR2 $406.02 .02 /$ SR2 $506.02 .02 /$ SR2 $606.02 .02 /$ SR2 7

$06.02 .02 /$ SR2 $806.02 .02 /$ SR29

\#F 219 <inquire hlhsr mode>:

04.03 .03 /SR05 04.03.03/SR06 04.03.03/SR07 $04.03 .03 /$ SRII

$04.03 .03 /$ SR12 $04.03 .03 /$ SR13 $04.03 .03 /$ SR14 $04.03 .03 /$ SR15

\#F 220 <inquire posted structures>:

$05.03 /$ SR13 05.03/SR14

\#F 221 <inquire display update state>:

04.03.03/SR06 04.03.03/SR07 05.02/SR01 05.02/SR02 05.02/SR04

$05.02 /$ SR11 05.02/SR12 $05.02 /$ SR13 $05.02 /$ SR14 $05.02 /$ SR15 $05.02 / S R 22$

$05.02 /$ SR2 $305.02 /$ SR24 $05.02 /$ SR2 $505.02 /$ SR2 $605.02 /$ SR2 $705.02 /$ SR2 8

$05.02 /$ SR2 $905.02 /$ SR31 $05.02 /$ SR32 $06.02 .02 /$ SR2 $706.02 .02 /$ SR2 8

$06.03 /$ SR18 $06.03 /$ SR19

\#F 222 <inquire list of polyline indices>:

$04.02 .01 .02 /$ SR12 $04.02 .01 .02 /$ SR13 $04.02 .01 .02 /$ SR16

$04.02 .01 .02 / S R 2404.02 .01 .02 / S R 25$

\#F 223 <inquire polyline representation>:

$04.02 .01 .02 /$ SR13 $04.02 .01 .02 /$ SR14 04.02.01.02/SR15

$04.02 .01 .02 /$ SR16 $04.02 .01 .02 /$ SR2 $404.02 .01 .02 /$ SR2 5

$04.02 .01 .02 /$ SR $4804.02 .01 .02 /$ SR 49

\#F 224 <inquire list of polymarker indices>:

$04.02 .02 .02 /$ SR12 $04.02 .02 .02 /$ SR13 $04.02 .02 .02 /$ SR16

$04.02 .02 .02 /$ SR2 $404.02 .02 .02 /$ SR2 5

\#F 225 <inquire polymarker representation>:

$04.02 .02 .02 /$ SR13 $04.02 .02 .02 /$ SR14 $04.02 .02 .02 /$ SR15

$04.02 .02 .02 /$ SR16 $04.02 .02 .02 /$ SR2 $404.02 .02 .02 /$ SR2 5

$04.02 .02 .02 /$ SR $4904.02 .02 .02 /$ SR 50 
\#F 226 <inquire list of text indices>:

$04.02 .03 .02 /$ SR12 $04.02 .03 .02 /$ SR13 $04.02 .03 .02 /$ SR16

$04.02 .03 .02 /$ SR2 $404.02 .03 .02 /$ SR2 $504.02 .04 .02 /$ SR0 9

\#F 227 <inquire text representation>:

$04.02 .03 .02 /$ SR13 $04.02 .03 .02 /$ SR14 $04.02 .03 .02 /$ SR15

$04.02 .03 .02 /$ SR16 $04.02 .03 .02 /$ SR24 $04.02 .03 .02 /$ SR2 5

$04.02 .03 .02 /$ SR2 $804.02 .03 .02 /$ SR3 $4 \quad 04.02 .03 .02 /$ SR3 5

$04.02 .03 .02 /$ SR $4704.02 .03 .02 /$ SR55 $04.02 .03 .02 /$ SR 65

$04.02 .03 .02 /$ SR66 $04.02 .04 .02 /$ SR09 $04.02 .04 .02 /$ SR10

$04.02 .04 .02 /$ SR20 $04.02 .04 .02 /$ SR30 $04.02 .04 .02 /$ SR 31

\#F 228 <inquire list of interior indices>:

$04.02 .05 .02 /$ SR13 $04.02 .05 .02 /$ SR14 04.02.05.02/SR17

$04.02 .05 .02 /$ SR25 $04.02 .05 .02 /$ SR2 6

\#F 229 <inquire interior representation>:

$04.02 .05 .02 /$ SR14 $04.02 .05 .02 /$ SR15 $04.02 .05 .02 /$ SR16

$04.02 .05 .02 /$ SR17 $04.02 .05 .02 /$ SR2 $504.02 .05 .02 / S R 26$

$04.02 .05 .02 /$ SR31 $04.02 .05 .02 /$ SR40 $04.02 .05 .02 / S R 41$

$04.02 .05 .02 /$ SR52 $04.02 .05 .02 /$ SR5 3

\#F 230 <inquire list of edge indices>:

$04.02 .05 .03 /$ SR12 $04.02 .05 .03 /$ SR13 $04.02 .05 .03 /$ SR16

$04.02 .05 .03 /$ SR2 $404.02 .05 .03 / S R 25$

\#F 231 <inquire edge representation>:

$04.02 .05 .03 /$ SR13 $04.02 .05 .03 /$ SR14 $04.02 .05 .03 /$ SR15

$04.02 .05 .03 /$ SR16 $04.02 .05 .03 /$ SR24 $04.02 .05 .03 /$ SR2 5

$04.02 .05 .03 /$ SR29 $04.02 .05 .03 /$ SR51 $04.02 .05 .03 /$ SR52

\#F 232 <inquire list of pattern indices>:

$04.02 .05 .01 /$ SR20 $04.02 .05 .01 /$ SR21 $04.02 .05 .04 /$ SR03

$04.02 .05 .04 /$ SR0 $404.02 .05 .04 /$ SR $0704.02 .05 .04 /$ SR 16

$04.02 .05 .04 /$ SR 17

\#F 233 <inquire pattern representation>:

$04.02 .05 .01 /$ SR20 04.02.05.04/SR04 04.02.05.04/SR0 5

$04.02 .05 .04 /$ SR06 $04.02 .05 .04 /$ SR0 $704.02 .05 .04 /$ SR16

$04.02 .05 .04 /$ SR17 $04.02 .05 .04 /$ SR2 $0 \quad 04.02 .05 .04 /$ SR2 1

\#F 234 <inquire colour model>:

$04.03 .02 .01 /$ SR10 $04.03 .02 .02 /$ SR17 $04.03 .02 .02 /$ SR2 0

\#F 235 <inquire list of colour indices > :

$04.02 .01 .02 /$ SR4 $804.02 .01 .02 /$ SR $4904.02 .02 .02 /$ SR 49

$04.02 .02 .02 /$ SR50 $04.02 .03 .02 /$ SR65 $04.02 .03 .02 /$ SR 66

$04.02 .04 .02 /$ SR30 $04.02 .04 .02 /$ SR31 $04.02 .05 .02 /$ SR52

$04.02 .05 .02 /$ SR53 $04.02 .05 .03 /$ SR51 $04.02 .05 .03 /$ SR52

$04.03 .02 .01 /$ SR03 $04.03 .02 .01 /$ SR0 $404.03 .02 .01 /$ SR0 6

$04.03 .02 .02 /$ SR13 $04.03 .02 .02 /$ SR14

\#F 236 <inquire colour representation>: 


$\begin{array}{llll}04.03 .02 .01 / \text { SR0 } 4 & 04.03 .02 .01 / \text { SR0 } 5 & 04.03 .02 .01 / \text { SR0 } 6 \\ 04.03 .02 .02 / \text { SR03 } & 04.03 .02 .02 / \text { SR0 } & 04.03 .02 .02 / \text { SR0 } \\ 04.03 .02 .02 / \text { SR13 } & 04.03 .02 .02 / \text { SR14 } & 04.03 .02 .02 / \text { SR2 } 1 \\ 04.03 .02 .02 / \text { SR26 } & 04.03 .02 .02 / \text { SR30 } & 04.03 .02 .02 / \text { SR31 } \\ 04.03 .02 .02 / \text { SR32 } & 04.03 .02 .02 / \text { SR33 } & \end{array}$

\#F 237 <inquire highlighting filter>:

$04.03 .04 .02 /$ SR02 $04.03 .04 .02 /$ SR03

\#F 238 <inquire invisibility filter>:

$04.03 .04 .02 /$ SR0 $04.03 .04 .02 /$ SR0 7

\#F 239 <inquire workstation transformation 3>:

$06.03 /$ SR12 06.03/SR15 06.03/SR16 06.03/SR17 06.03/SR18 06.03/SR19 $06.03 /$ SR2 0

\#F 240 <inquire workstation transformation>:

$06.03 /$ SR13 06.03/SR15 06.03/SR16 06.03/SR17 06.03/SR18 06.03/SR19 $06.03 /$ SR2 0

\#F 253 <inquire workstation category>:

05.01.02/SR01 05.01.02/SR02 05.01.02/SR03 05.01.02/SR04

$05.01 .02 /$ SR10

\#F 254 <inquire display space size 3>:

$05.01 .02 /$ SR01 $06.03 /$ SR02 $06.03 /$ SR04

\#F 255 <inquire display space size>:

$04.01 /$ SR02 05.01.02/SR01 06.03/SR03 06.03/SR0 4

\#F 256 <inquire hlhsr facilities>:

$04.03 .03 /$ SR0 $84.03 .03 /$ SR09 $04.03 .03 /$ SR10 $04.03 .03 /$ SRII

$05.01 .02 /$ SRO1

\#F 257 <inquire view facilities>:

$05.01 .02 /$ SR01 $06.02 .02 /$ SR0 $406.02 .02 /$ SR0 5

\#F 258 <inquire predefined view representation>:

05.01.02/SR01 06.02.02/SR06 06.02.02/SR07 06.02.02/SR09

\#F 259 <inquire workstation classification>:

$05.01 .02 /$ SR01 $05.01 .02 /$ SR09 $05.01 .02 /$ SR10

\#F 260 <inquire dynamics of workstation attributes >

$04.03 .03 /$ SR06 04.03.03/SR07 05.01.02/SR01 05.02/SR06 05.02/SR19

$05.02 / S R 2005.02 / S R 2105.02 / S R 2405.02 / S R 2505.02 / S R 2605.02 / S R 27$

$05.02 /$ SR2 $805.02 /$ SR2 $905.02 /$ SR31 $05.02 /$ SR32

\#F 261 <inquire default display update state>:

$05.01 .02 /$ SR01 $05.02 /$ SR01 05.02/SR02 05.02/SR0 5

\#F 262 <inquire polyline facilities>:

$04.02 .01 .01 /$ SR06 $04.02 .01 .01 /$ SR0 $04.02 .01 .01 /$ SR 15

$04.02 .01 .01 /$ SR16 $04.02 .01 .02 /$ SR19 $04.02 .01 .02 /$ SR21 
$04.02 .01 .02 / S R 25$

$04.02 .01 .02 /$ SR29

$04.02 .01 .02 /$ SR 32

$04.02 .01 .02 /$ SR39
$04.02 .01 .02 / \mathrm{SR} 26$

$04.02 .01 .02 / S R 30$

$04.02 .01 .02 /$ SR 33

$04.02 .01 .02 /$ SR 40
$04.02 .01 .02 /$ SR2 7

$04.02 .01 .02 / \mathrm{SR} 31$

$04.02 .01 .02 / \mathrm{SR} 34$

$04.02 .01 .02 /$ SR4 $105.01 .02 /$ SR01

\#F 263 <inquire predefined polyline representation>:

$04.02 .01 .02 /$ SR20 $04.02 .01 .02 /$ SR21 $04.02 .01 .02 /$ SR2 2

$04.02 .01 .02 /$ SR23 $04.02 .01 .02 / S R 2404.02 .01 .02 / S R 26$

$04.02 .01 .02 /$ SR2 $704.02 .01 .02 /$ SR2 8 05.01.02/SR01

\#F 264 <inquire polymarker facilities>:

$04.02 .02 .01 /$ SR0 $64.02 .02 .01 /$ SR0 $704.02 .02 .01 /$ SR15

$04.02 .02 .01 /$ SR16 $04.02 .02 .02 /$ SR19 $04.02 .02 .02 /$ SR2 1

$04.02 .02 .02 /$ SR2 $504.02 .02 .02 /$ SR2 $604.02 .02 .02 /$ SR2 7

$04.02 .02 .02 /$ SR29 $04.02 .02 .02 /$ SR30 $04.02 .02 .02 /$ SR 31

$04.02 .02 .02 /$ SR $3204.02 .02 .02 /$ SR3 $304.02 .02 .02 /$ SR 34

$04.02 .02 .02 /$ SR39 $04.02 .02 .02 /$ SR40 $04.02 .02 .02 /$ SR $4105.01 .02 /$ SR01

\#F 265 <inquire predefined polymarker representation>:

$04.02 .02 .02 /$ SR20 $04.02 .02 .02 /$ SR21 $04.02 .02 .02 /$ SR2 2

$04.02 .02 .02 /$ SR23 $04.02 .02 .02 /$ SR2 $404.02 .02 .02 / S R 26$

$04.02 .02 .02 /$ SR2 $704.02 .02 .02 /$ SR2 $805.01 .02 /$ SR01

\#F 266 <inquire text facilities>:

$04.02 .03 .01 /$ SR21 $04.02 .03 .01 /$ SR22

$04.02 .03 .01 /$ SR $3704.02 .03 .01 /$ SR5 3

$04.02 .03 .01 /$ SR55 $04.02 .03 .01 /$ SR5 6

$04.02 .03 .01 /$ SR $6104.02 .03 .02 /$ SR19

$04.02 .03 .02 /$ SR2 $504.02 .03 .02 /$ SR2 6

$04.02 .03 .02 /$ SR3 $004.02 .03 .02 /$ SR3 1

$04.02 .03 .02 /$ SR $3304.02 .03 .02 /$ SR 34

$04.02 .03 .02 /$ SR $3604.02 .03 .02 /$ SR 37

$04.02 .03 .02 /$ SR $4704.02 .03 .02 /$ SR5 1

$04.02 .03 .02 /$ SR5 $304.02 .03 .02 /$ SR 54

$04.02 .03 .02 /$ SR59 $04.02 .04 .01 /$ SR14

$04.02 .04 .01 /$ SR20 $04.02 .04 .01 /$ SR29

$04.02 .04 .02 /$ SR10 $04.02 .04 .02 /$ SR13

$04.02 .04 .02 /$ SR2 $605.01 .02 /$ SR01

$04.02 .03 .01 /$ SR2 8

$04.02 .03 .01 /$ SR5 4

$04.02 .03 .01 /$ SR 57

$04.02 .03 .02 /$ SR 21

$04.02 .03 .02 /$ SR 27

$04.02 .03 .02 /$ SR 32

$04.02 .03 .02 /$ SR 35

$04.02 .03 .02 /$ SR 40

$04.02 .03 .02 /$ SR 52

$04.02 .03 .02 /$ SR 55

$04.02 .04 .01 /$ SR15

$04.02 .04 .01 /$ SR 52

$04.02 .04 .02 / S R 20$

\#F 267 <inquire predefined text representation>:

$04.02 .03 .02 /$ SR20 $04.02 .03 .02 /$ SR21 $04.02 .03 .02 /$ SR2 2

$04.02 .03 .02 /$ SR23 $04.02 .03 .02 /$ SR2 $404.02 .03 .02 /$ SR2 6

$04.02 .03 .02 /$ SR2 $704.02 .03 .02 / S R 2804.02 .03 .02 /$ SR29

$04.02 .03 .02 /$ SR $3404.02 .04 .02 /$ SR0 $804.02 .04 .02 /$ SR0 9

$04.02 .04 .02 /$ SR10 $05.01 .02 /$ SR01

\#F 268 <inquire annotation facilities>:

$04.02 .04 .01 /$ SR43 $04.02 .04 .01 /$ SR4 $404.02 .04 .01 /$ SR 45

$04.02 .04 .01 /$ SR $4604.02 .04 .01 /$ SR $4704.02 .04 .01 / S R 77$

$04.02 .04 .01 /$ SR78 $04.02 .04 .01 /$ SR79 $04.02 .04 .01 /$ SR80

$04.02 .04 .01 /$ SR81 $04.02 .04 .01 /$ SR82 $05.01 .02 /$ SR01

\#F 269 <inquire text extent> :

$04.02 .03 .01 /$ SR3 $604.02 .03 .01 /$ SR4 $204.02 .03 .01 /$ SR59 

$04.02 .03 .02 /$ SR5 8
$04.02 .03 .03 /$ SR 04
$04.02 .03 .03 /$ SR 07
$04.02 .03 .03 /$ SR 10
$04.02 .03 .03 /$ SR13
$04.02 .03 .03 /$ SR16
$04.02 .04 .01 /$ SR2 7
$04.02 .04 .02 /$ SR 25
$04.02 .03 .02 /$ SR 62
$04.02 .03 .03 /$ SR0 5
$04.02 .03 .03 /$ SR0 8
$04.02 .03 .03 /$ SRII
$04.02 .03 .03 /$ SR 14
$04.02 .03 .03 /$ SR 17
$04.02 .04 .01 /$ SR 33
$04.02 .03 .03 /$ SR01
$04.02 .03 .03 /$ SR0 6
$04.02 .03 .03 /$ SR09
$04.02 .03 .03 /$ SR12
$04.02 .03 .03 /$ SR 15
$04.02 .03 .03 /$ SR1 8
$04.02 .04 .01 /$ SR 49
$04.02 .04 .02 /$ SR2 $805.01 .02 /$ SR01

\#F 270 <inquire interior facilities>:

$04.02 .05 .01 /$ SR08 $04.02 .05 .01 /$ SR09 $04.02 .05 .01 /$ SR2 0

$04.02 .05 .01 /$ SR21 $04.02 .05 .02 /$ SR2 $004.02 .05 .02 /$ SR2 2

$04.02 .05 .02 /$ SR2 $604.02 .05 .02 /$ SR2 $704.02 .05 .02 /$ SR2 9

$04.02 .05 .02 /$ SR3 $104.02 .05 .02 /$ SR $3204.02 .05 .02 /$ SR 33

$04.02 .05 .02 /$ SR3 $404.02 .05 .02 /$ SR $4204.02 .05 .02 /$ SR 43

$04.02 .05 .02 /$ SR44 $04.02 .05 .02 /$ SR $4504.02 .05 .02 /$ SR 46

$04.02 .05 .02 /$ SR $4705.01 .02 /$ SR01

\#F 271 <inquire predefined interior representation>:

$04.02 .05 .02 /$ SR21 $04.02 .05 .02 /$ SR2 $204.02 .05 .02 /$ SR23

$04.02 .05 .02 /$ SR2 $404.02 .05 .02 /$ SR2 $504.02 .05 .02 /$ SR2 7

$04.02 .05 .02 /$ SR2 $804.02 .05 .02 /$ SR2 $904.02 .05 .02 /$ SR 30

$04.02 .05 .02 /$ SR $3105.01 .02 /$ SR01

\#F 272 <inquire edge facilities>:

$04.02 .05 .01 /$ SR42 $04.02 .05 .01 /$ SR43 $04.02 .05 .01 /$ SR5 1

$04.02 .05 .01 /$ SR52 $04.02 .05 .03 /$ SR19 $04.02 .05 .03 /$ SR21

$04.02 .05 .03 /$ SR2 $604.02 .05 .03 /$ SR2 $704.02 .05 .03 /$ SR 32

$04.02 .05 .03 /$ SR33 $04.02 .05 .03 /$ SR3 $404.02 .05 .03 /$ SR3 5

$04.02 .05 .03 /$ SR3 $64.02 .05 .03 /$ SR3 $704.02 .05 .03 /$ SR 42

$04.02 .05 .03 /$ SR43 $04.02 .05 .03 /$ SR $4405.01 .02 /$ SR01

\#F 273 <inquire predefined edge representation>:

$04.02 .05 .03 /$ SR20 $04.02 .05 .03 /$ SR21 $04.02 .05 .03 /$ SR2 2

$04.02 .05 .03 /$ SR23 $04.02 .05 .03 /$ SR2 $404.02 .05 .03 /$ SR2 5

$04.02 .05 .03 /$ SR2 $604.02 .05 .03 /$ SR2 $704.02 .05 .03 /$ SR2 8

$04.02 .05 .03 /$ SR29 $05.01 .02 /$ SR01

\#F 274 <inquire pattern facilities>:

$04.02 .05 .02 /$ SR2 $804.02 .05 .02 /$ SR34 $04.02 .05 .04 /$ SR11

$04.02 .05 .04 /$ SR13 $04.02 .05 .04 /$ SR14 $05.01 .02 /$ SR01

\#F 275 <inquire predefined pattern representation>: $04.02 .05 .04 /$ SR12 $04.02 .05 .04 /$ SR13 $04.02 .05 .04 /$ SR15

$04.02 .05 .04 /$ SR16 $04.02 .05 .04 /$ SRI7 $04.02 .05 .04 /$ SRI8 $05.01 .02 /$ SR01

\#F 276 <inquire colour model facilities>:

$04.03 .02 .02 /$ SR15 $04.03 .02 .02 /$ SR16 $04.03 .02 .02 /$ SR17

$04.03 .02 .02 /$ SR18 $04.03 .02 .02 /$ SR19 $04.03 .02 .02 /$ SR2 0

$04.03 .02 .02 /$ SR2 $204.03 .02 .02 /$ SR23 $04.03 .02 .02 /$ SR 24

$04.03 .02 .02 /$ SR2 $504.03 .02 .02 /$ SR28 $04.03 .02 .02 /$ SR2 $905.01 .02 /$ SR01

\#F 277 <inquire colour facilities>:

$04.02 .01 .02 /$ SR2 $804.02 .02 .02 /$ SR2 $804.02 .03 .02 /$ SR2 9 


\begin{abstract}
$04.02 .05 .02 /$ SR30 $04.02 .05 .03 /$ SR2 $804.02 .05 .04 / S R 18$
$04.03 .02 .02 /$ SR01 $04.03 .02 .02 /$ SR02 $04.03 .02 .02 / S R 03$

$04.03 .02 .02 /$ SR0 $404.03 .02 .02 /$ SR0 $504.03 .02 .02 /$ SR0 6

$04.03 .02 .02 /$ SR0 $704.03 .02 .02 /$ SR0 $804.03 .02 .02 /$ SR 10

$04.03 .02 .02 /$ SRI1 $05.01 .02 /$ SR01
\end{abstract}

\#F 278 <inquire predefined colour representation>:

04.03 .02 .02 /SR09 $04.03 .02 .02 /$ SR10 $04.03 .02 .02 /$ SR11

$04.03 .02 .02 /$ SR12 $04.03 .02 .02 /$ SRI3 $04.03 .02 .02 /$ SRI4

$04.03 .02 .02 /$ SRI5 $05.01 .02 /$ SR01

\#F 279 <inquire list of available generalized drawing primitives 3>: $04.01 .08 /$ SR0 $405.01 .02 /$ SR01

\#F 280 <inquire list of available generalized drawing primitives>: $04.01 .08 /$ SR09 $05.01 .02 /$ SR01

\#F 281 <inquire generalized drawing primitive 3>:

$04.01 .08 /$ SR05 05.01.02/SR01

\#F 282 <inquire generalized drawing primitive>:

$04.01 .08 /$ SR10 05.01.02/SR01

\#F 283 <inquire list of available generalized structure elements>: $02.02 .02 /$ SRI1 $02.02 .02 /$ SR13 $05.01 .02 /$ SR01

\#F 284 <inquire number of display priorities supported>:

$05.01 .02 /$ SR01 $05.03 /$ SRI5 05.03/SR16 05.03/SR17 05.03/SR18

\#F 285 <inquire workstation state table lengths>:

$04.02 .01 .02 /$ SR17 $04.02 .01 .02 /$ SR18 $04.02 .02 .02 /$ SR17

$04.02 .02 .02 /$ SR18 $04.02 .03 .02 /$ SR17 $04.02 .03 .02 /$ SR18

$04.02 .05 .02 /$ SR18 $04.02 .05 .02 /$ SR19 $04.02 .05 .03 /$ SRI7

$04.02 .05 .03 /$ SR18 $04.02 .05 .04 /$ SR08 $04.02 .05 .04 /$ SR0 9

$04.02 .05 .04 /$ SRI0 $04.03 .02 .01 /$ SR0 $704.03 .02 .01 /$ SR0 $805.01 .02 /$ SR01

$06.02 .02 /$ SR12 $06.02 .02 /$ SR13

\#F 286 <inquire dynamics of structures>:

05.01.02/SR01 05.02/SR07 05.02/SR19 05.02/SR20 05.02/SR21

$05.02 /$ SR2 $405.02 /$ SR2 $505.02 /$ SR2 $605.02 / S R 2705.02 / S R 2805.02 / S R 29$

$05.02 /$ SR31 $05.02 /$ SR32

\#F 287 <inquire number of available logical input devices>:

$05.01 .02 /$ SR01

\#F 288 <inquire default locator device data 3>:

05.01 .02 /SR01

\#F 289 <inquire default locator device data>:

$05.01 .02 /$ SR01

\#F 290 <inquire default stroke device data 3>:

$05.01 .02 /$ SR01 
FUNCTION CROSS-REFERENCE

\#F 291 <inquire default stroke device data>:

$05.01 .02 /$ SR01

\#F 292 <inquire default valuator device data 3>:

$05.01 .02 /$ SR01

\#F 293 <inquire default valuator device data>:

$05.01 .02 /$ SR01

\#F 294 <inquire default choice device data 3>:

$05.01 .02 /$ SR01

\#F 295 <inquire default choice device data>:

$05.01 .02 /$ SR01

\#F 296 <inquire default pick device data 3>:

$05.01 .02 /$ SR01

\#F 297 <inquire default pick device data>:

05.01 .02 /SR01

\#F 298 <inquire default string device data $3>$ :

05.01.02/SR01

\#F 299 <inquire default string device data>:

$05.01 .02 /$ SR01

\#F 300 <inquire set of workstations to which posted>: $05.03 /$ SR12

\#F 301 <inquire open structures:

$02.02 .01 /$ SR05 02.02.01/SR06

\#F 302 <inquire element pointer>:

$02.02 .01 /$ SR0 8

\#F 303 <inquire current element type and size>:

$02.02 .02 /$ SR02 $02.02 .02 /$ SR05 $02.02 .02 /$ SR09 $02.03 .01 /$ SR01

$02.03 .01 /$ SR02 $04.01 .01 /$ SR02 $04.01 .01 /$ SR0 $504.01 .02 /$ SR02

$04.01 .02 /$ SR05 $04.01 .03 /$ SR02 $04.01 .03 /$ SR05 $04.01 .04 /$ SR02

$04.01 .04 /$ SR05 $04.01 .05 /$ SR02 $04.01 .05 /$ SR05 $04.01 .06 /$ SR02

$04.01 .06 /$ SR05 $04.01 .07 /$ SR02 $04.01 .07 /$ SR07 $04.01 .08 /$ SR02

$04.01 .08 /$ SR0 $04.02 .01 .01 /$ SR01 04.02.01.02/SR01 04.02.02.01/SR01

$04.02 .02 .02 /$ SR01 $04.02 .03 .01 /$ SR09 $04.02 .03 .02 /$ SR01

$04.02 .04 .01 /$ SR03 $04.02 .05 .01 /$ SR01 $04.02 .05 .02 /$ SR01

$04.02 .05 .03 /$ SR01 $04.03 .01 /$ SR02 $04.03 .03 /$ SRO2 $04.03 .04 .01 /$ SR02

$04.03 .04 .01 /$ SR0 $506.01 .02 /$ SR02 $06.01 .02 /$ SR05 $06.01 .02 /$ SR10

$06.01 .02 /$ SR13 06.01.02/SR25 $06.01 .02 /$ SR28 $06.01 .02 /$ SR31

$06.01 .02 /$ SR34 $06.02 .02 /$ SR02

\#F 304 <inquire current element content>:

$02.02 .02 /$ SR03 02.02.02/SR06 $02.02 .02 /$ SR10 $02.03 .01 /$ SR03

$04.01 .01 /$ SR03 $04.01 .01 /$ SR0 $604.01 .02 /$ SR03 $04.01 .02 /$ SR0 6

$04.01 .03 /$ SR03 04.01.03/SR0 $64.01 .04 /$ SR03 $04.01 .04 /$ SR0 6 
$04.01 .05 /$ SR03 04.01.05/SR06 04.01.06/SR03 04.01.06/SR06

$04.01 .07 /$ SR03 04.01.07/SR08 04.01.08/SR03 04.01.08/SR08

$04.02 .01 .01 /$ SR02 $04.02 .01 .02 /$ SR02 $04.02 .02 .01 /$ SR02

$04.02 .02 .02 /$ SR02 $04.02 .03 .01 /$ SR10 $04.02 .03 .02 /$ SR02

$04.02 .04 .01 /$ SR0 $404.02 .05 .01 /$ SR02 $04.02 .05 .02 / S R 02$

$04.02 .05 .03 /$ SR02 $04.03 .01 /$ SR03 $04.03 .03 /$ SR03 $04.03 .04 .01 /$ SR03

$04.03 .04 .01 /$ SR0 $606.01 .02 /$ SR03 06.01.02/SR06 06.01.02/SR11

$06.01 .02 /$ SR14 06.01.02/SR26 $06.01 .02 /$ SR29 $06.01 .02 /$ SR32

$06.01 .02 /$ SR3 $506.02 .02 /$ SR03

\#F 305 <inquire element type and size>:

$02.03 .01 /$ SR04 $02.03 .01 /$ SR0 5

\#F 306 <inquire element content>:

$02.03 .01 /$ SR06

\#F 307 <inquire structure status>:

$02.02 .01 /$ SR0 7

\#F 308 <inquire paths to ancestors>:

$02.01 /$ SR03 02.01/SR05 02.01/SR06 02.01/SR07 02.01/SR09 02.01/SR10

$02.01 /$ SR13

\#F 309 <inquire paths to descendants > :

$02.01 /$ SR0 $402.01 /$ SR05 $02.01 /$ SR0 $02.01 / S R 0802.01 / S R 1102.01 / S R 12$

$02.01 /$ SR 14

\#F 310 <element search>:

02.03 .02 /SR01 $02.03 .02 /$ SR02 $02.03 .02 /$ SR03 $02.03 .02 /$ SR0 4

$02.03 .02 /$ SR05 $02.03 .02 /$ SR0 $62.03 .02 /$ SR0 $02.03 .02 /$ SR0 8

\#F 311 <incremental spatial search 3>:

$02.03 .03 /$ SR01 02.03.03/SR02 02.03.03/SR03 $02.03 .03 /$ SR0 4

$02.03 .03 /$ SR05 $02.03 .03 /$ SR06 $02.03 .03 /$ SR07 $02.03 .03 /$ SR0 8

$02.03 .03 /$ SR09 $02.03 .03 /$ SR10 $02.03 .03 /$ SR11 $02.03 .03 /$ SR 12

$02.03 .03 /$ SR13 $02.03 .03 /$ SR14 $02.03 .03 /$ SR15 $02.03 .03 /$ SR16

$02.03 .03 /$ SR17 $02.03 .03 /$ SR18 $02.03 .03 /$ SR19 $02.03 .03 /$ SR2 0

$02.03 .03 /$ SR22 $06.01 .02 /$ SR20 $06.01 .02 / S R 43$

\#F 312 <incremental spatial search>:

$02.03 .03 /$ SR01 $02.03 .03 /$ SR02 $02.03 .03 /$ SR03 $02.03 .03 /$ SR0 4

$02.03 .03 /$ SR0 $502.03 .03 /$ SR0 $62.03 .03 /$ SR0 $0202.03 .03 /$ SR0 8

$02.03 .03 /$ SR09 $02.03 .03 /$ SR10 $02.03 .03 /$ SR11 $02.03 .03 /$ SR12

$02.03 .03 /$ SR13 $02.03 .03 /$ SR14 $02.03 .03 /$ SR15 $02.03 .03 /$ SR16

$02.03 .03 /$ SR17 $02.03 .03 /$ SR18 $02.03 .03 /$ SR19 $02.03 .03 /$ SR2 0

$02.03 .03 /$ SR22 $06.01 .02 /$ SR2 $006.01 .02 / S R 43$

\#F 320 <pack data record>:

$11 /$ SR01

\#F 321 <unpack data record>:

$11 /$ SR02 
APPENDIX H

DATA STRUCTURE CROSS-REFERENCE

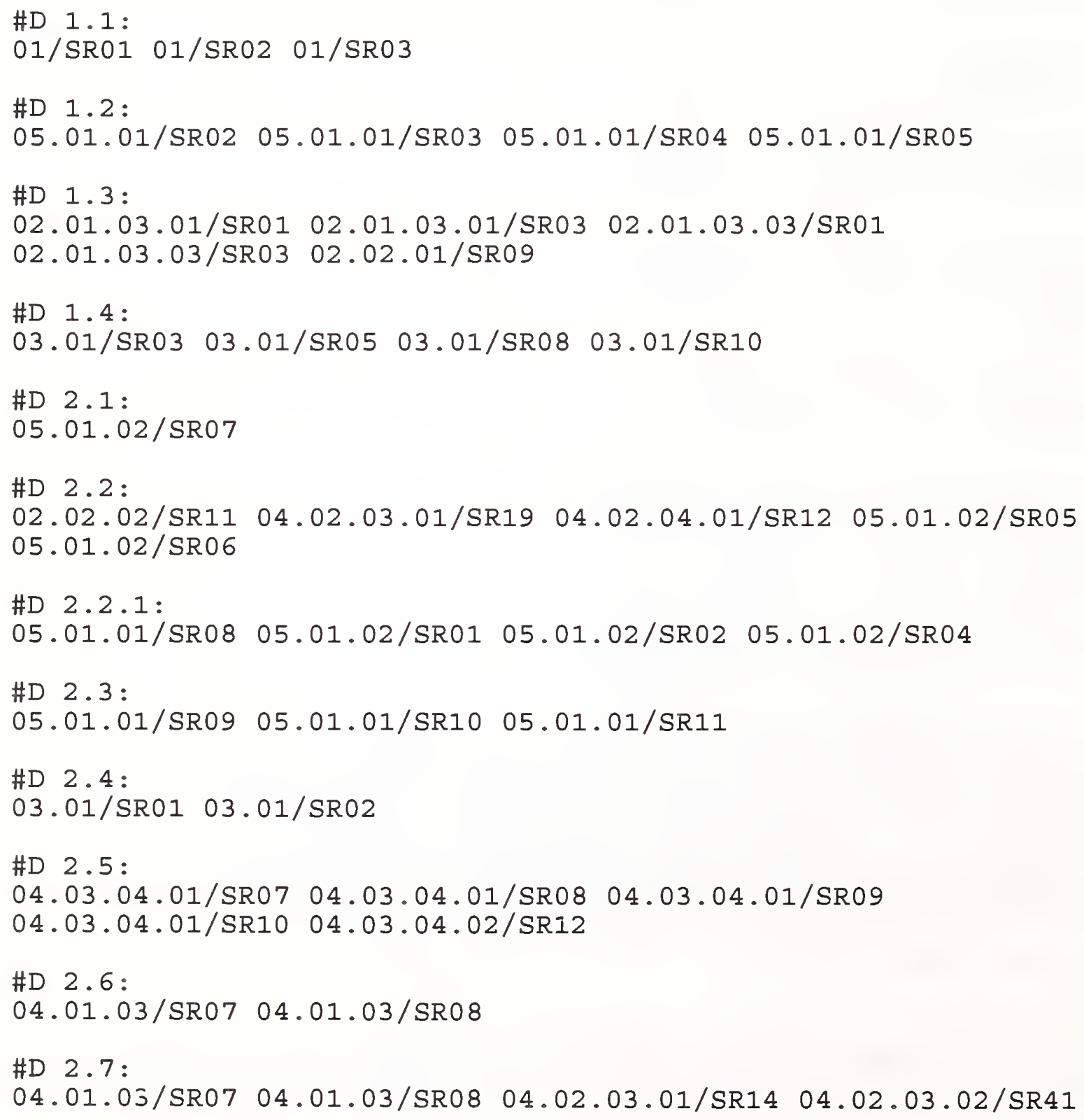


$04.02 .04 .01 /$ SR07 $04.02 .04 .02 /$ SR14

\#D 2.8:

$02.03 .03 /$ SR21 $02.03 .03 /$ SR2 2

\#D $2.9:$

$02.03 .03 /$ SR21 $02.03 .03 /$ SR 22

\#D 2.10.1:

$04.02 .01 .02 /$ SR0 8

\#D 2.10.2:

$04.02 .01 .01 /$ SR 11

\#D 2.10.3:

$04.02 .01 .01 /$ SR 17

\#D 2.10.4:

$04.02 .01 .01 /$ SR23

\#D 2.10.5:

$04.03 .01 /$ SR 10

\#D 2.10.6:

$04.03 .01 /$ SR10

\#D 2.10.7:

$04.03 .01 /$ SR 10

\#D 2.11.1:

$04.02 .02 .02 /$ SR0 8

\#D 2.11.2:

$04.02 .02 .01 /$ SRI1

\#D 2.11.3:

$04.02 .02 .01 /$ SR 18

\#D 2.11.4:

$04.02 .02 .01 /$ SR2 4

\#D 2.11.5:

$04.03 .01 /$ SR10

\#D 2.11.6:

$04.03 .01 /$ SR 10

\#D 2.11.7:

$04.03 .01 /$ SR 10

\#D 2.12.1:

$04.02 .03 .02 /$ SR08 $04.02 .04 .02 /$ SR0 6

\#D 2.12.2: 


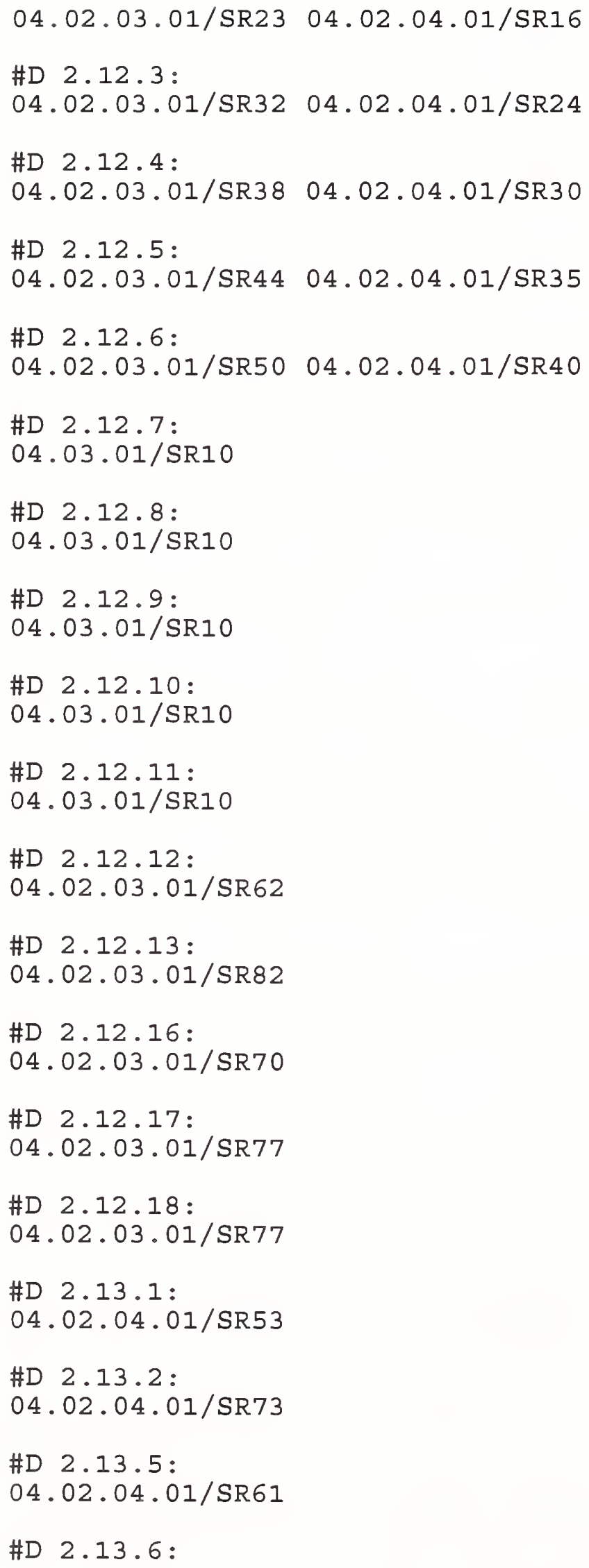


$04.02 .04 .01 /$ SR 68

\#D 2.13.7:

$04.02 .04 .01 /$ SR 68

\#D 2.13.8:

$04.02 .04 .01 /$ SR8 3

\#D 2.14.1:

$04.02 .05 .02 /$ SRO9

\#D 2.14 .2 :

$04.02 .05 .01 /$ SR1 6

\#D 2.14.3:

$04.02 .05 .01 /$ SR2 4

\#D 2.14.4:

$04.02 .05 .01 /$ SR 30

\#D 2.14.5:

$04.03 .01 /$ SR10

\#D 2.14.6:

$04.03 .01 /$ SR 10

\#D 2.14.7:

$04.03 .01 /$ SR 10

\#D 2.15.1:

$04.02 .05 .03 /$ SR0 8

\#D 2.15.2:

$04.02 .05 .01 /$ SR 38

\#D 2.15.3:

$04.02 .05 .01 /$ SR 47

\#D 2.15.4:

$04.02 .05 .01 /$ SR5 3

\#D 2.15.5:

$04.02 .05 .01 /$ SR59

\#D 2.15.6:

$04.03 .01 /$ SR 10

\#D 2.15.7:

$04.03 .01 /$ SR10

\#D 2.15.8:

$04.03 .01 /$ SR 10

\#D 2.15.9: 


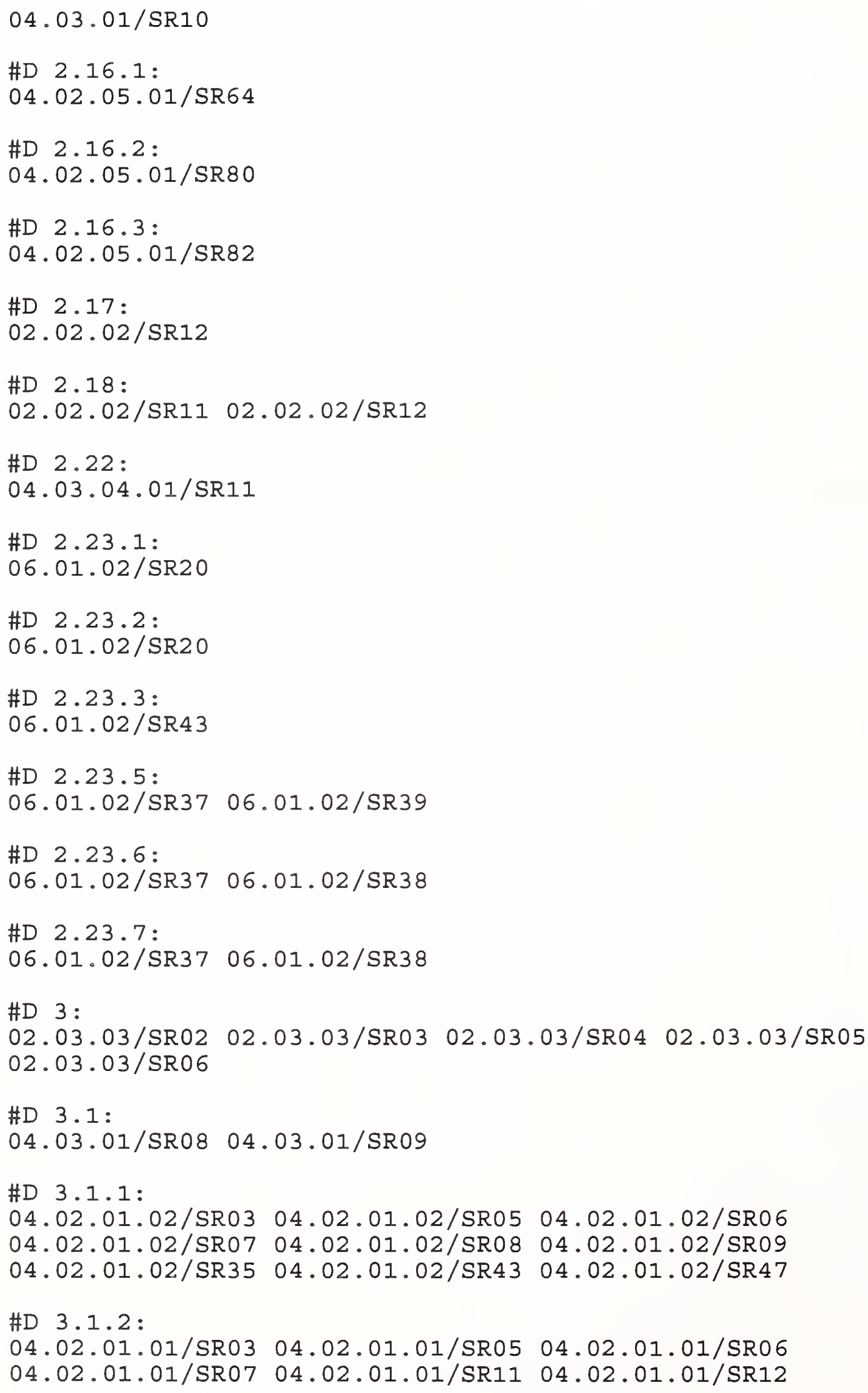


\#D 3.1.3:

$04.02 .01 .01 /$ SR03 $04.02 .01 .01 /$ SR14 $04.02 .01 .01 /$ SR15

$04.02 .01 .01 /$ SR16 $04.02 .01 .01 /$ SR17 $04.02 .01 .01 /$ SR18

\#D 3.1.4:

$04.02 .01 .01 /$ SR03 $04.02 .01 .01 /$ SR20 $04.02 .01 .01 / S R 21$

$04.02 .01 .01 / \mathrm{SR} 2204.02 .01 .01 / \mathrm{SR} 2304.02 .01 .01 / \mathrm{SR} 24$

\#D 3.1.5:

04.02.01.01/SR06 04.02.01.01/SR07 04.02.01.02/SR35 04.03.01/SR06 $04.03 .01 /$ SR0 $04.03 .01 /$ SR10 $04.03 .01 /$ SR11

\#D 3.1.6:

$04.02 .01 .01 /$ SR15 $04.02 .01 .01 /$ SR16 $04.02 .01 .02 /$ SR4 $304.03 .01 /$ SR0 6 $04.03 .01 /$ SR0 $04.03 .01 /$ SR10 $04.03 .01 /$ SR11

\#D $3.1 .7:$

$04.02 .01 .01 /$ SR2 $104.02 .01 .01 / S R 22 \quad 04.02 .01 .02 / S R 4704.03 .01 / S R 06$ $04.03 .01 /$ SR07 $04.03 .01 /$ SR10 $04.03 .01 /$ SR 11

\#D $3.2:$

$04.03 .01 /$ SR08 $04.03 .01 /$ SR09

\#D 3.2.1:

$04.02 .02 .02 /$ SR03 $04.02 .02 .02 /$ SR0 $04.02 .02 .02 /$ SR0 6

$04.02 .02 .02 /$ SR0 $704.02 .02 .02 /$ SR0 $804.02 .02 .02 /$ SR0 9

$04.02 .02 .02 /$ SR3 $504.02 .02 .02 /$ SR $4304.02 .02 .02 /$ SR 48

\#D $3.2 .2:$

$04.02 .02 .01 /$ SR03 $04.02 .02 .01 /$ SR0 $504.02 .02 .01 /$ SR0 6

$04.02 .02 .01 /$ SR0 $04.02 .02 .01 /$ SRI1 $04.02 .02 .01 /$ SR 12

\#D $3.2 .3:$

$04.02 .02 .01 /$ SR0 $304.02 .02 .01 /$ SR14 $04.02 .02 .01 /$ SR 15

$04.02 .02 .01 /$ SR16 $04.02 .02 .01 /$ SR18 $04.02 .02 .01 /$ SR19

\#D $3.2 .4:$

$04.02 .02 .01 /$ SR03 $04.02 .02 .01 /$ SR21 $04.02 .02 .01 /$ SR2 2

$04.02 .02 .01 /$ SR2 $304.02 .02 .01 /$ SR2 $4 \quad 04.02 .02 .01 /$ SR2 5

\#D $3.2 .5:$

$04.02 .02 .01 /$ SR06 $04.02 .02 .01 /$ SR07 $04.02 .02 .02 /$ SR35 $04.03 .01 /$ SR0 6 $04.03 .01 /$ SR0 $704.03 .01 /$ SR10 $04.03 .01 /$ SR 11

\#D $3.2 .6:$

$04.02 .02 .01 /$ SR15 $04.02 .02 .01 /$ SR16 $04.02 .02 .02 /$ SR $43 \quad 04.03 .01 /$ SR0 6 $04.03 .01 /$ SR0 $704.03 .01 /$ SRI0 $04.03 .01 /$ SRII

\#D 3.2.7:

$04.02 .02 .01 /$ SR22 $04.02 .02 .01 /$ SR2 $304.02 .02 .02 /$ SR4 $804.03 .01 /$ SR0 6 $04.03 .01 /$ SR0 $04.03 .01 /$ SR10 $04.03 .01 /$ SR 11

\#D $3.3:$

$04.03 .01 /$ SR08 $04.03 .01 /$ SR09 
\#D 3.3.1:

$04.02 .03 .02 /$ SR0 3

$04.02 .03 .02 /$ SR0 7

$04.02 .03 .02 /$ SR3 8

$04.02 .03 .02 /$ SR 61

$04.02 .04 .02 /$ SR0 3

$04.02 .04 .02 /$ SR0 6

$04.02 .04 .02 /$ SR 12

$04.02 .04 .02 /$ SR2 9

\#D $3.3 .2:$

$04.02 .03 .01 /$ SR11 $04.02 .03 .01 /$ SR22

$04.02 .03 .01 /$ SR2 8

$04.02 .04 .01 /$ SR1 4

$04.02 .04 .01 /$ SR 17

\#D $3.3 .3:$

$04.02 .03 .01 /$ SRI1

$04.02 .03 .01 /$ SR2 7

$04.02 .03 .01 /$ SR3 3

$04.02 .04 .01 /$ SR15

$04.02 .04 .01 /$ SR2 4

\#D 3.3 .4 :

$04.02 .03 .01 /$ SR 11

$04.02 .03 .01 /$ SR 38

$04.02 .04 .01 /$ SR2 6

$04.02 .04 .01 /$ SR31

\#D $3.3 .5:$

$04.02 .03 .01 /$ SR11 $04.02 .03 .01 / S R 44$

$04.02 .04 .01 /$ SR 32

$04.02 .04 .01 /$ SR3 6

$04.02 .03 .01 /$ SR 41 $04.02 .03 .01 /$ SR 45

$04.02 .04 .01 /$ SR 34

$04.02 .03 .01 /$ SR13

$04.02 .03 .01 /$ SR2 3

$04.02 .04 .01 /$ SR0 5

$04.02 .04 .01 /$ SR15

$04.02 .04 .01 /$ SR20

$04.02 .03 .01 / S R 21$

$04.02 .03 .01 /$ SR2 8

$04.02 .04 .01 /$ SR0 5

$04.02 .04 .01 /$ SR19

$04.02 .04 .01 /$ SR2 5

$04.02 .03 .01 /$ SR 35

$04.02 .03 .01 /$ SR3 9

$04.02 .04 .01 /$ SR29

$04.02 .03 .01 /$ SR 37

$04.02 .04 .01 /$ SR0 5

$04.02 .04 .01 /$ SR 30

$04.02 .03 .01 /$ SR22

$04.02 .03 .01 /$ SR 32

$04.02 .04 .01 /$ SR14

$04.02 .04 .01 /$ SR 20

\#D $3.3 .6:$

$04.02 .03 .01 /$ SR 11

$04.02 .03 .01 /$ SR 49

$04.02 .04 .01 /$ SR0 5

$04.02 .04 .01 /$ SR39

$04.02 .03 .01 / S R 47$

$04.02 .03 .01 /$ SR5 0

$04.02 .04 .01 /$ SR 37

$04.02 .04 .01 /$ SR 40

$04.02 .03 .01 / S R 43$

$04.02 .04 .01 /$ SR0 5

$04.02 .04 .01 /$ SR3 5

\#D $3.3 .7:$

$04.02 .03 .01 /$ SR21 $04.02 .03 .01 /$ SR2 2

$04.02 .03 .02 /$ SR $3804.02 .04 .01 /$ SR14

$04.02 .04 .01 /$ SR20 $04.02 .04 .02 /$ SR11

$04.03 .01 /$ SR $1004.03 .01 /$ SR11

\#D 3.3.8:

$04.02 .03 .01 / S R 2104.02 .03 .01 / S R 2204.02 .03 .01 / S R 28$

$04.02 .03 .02 /$ SR39 $04.02 .04 .01 /$ SR14 $04.02 .04 .01 /$ SR15

$04.02 .04 .01 /$ SR20 $04.02 .04 .02 /$ SR12 $04.03 .01 /$ SR0 $604.03 .01 /$ SR0 7

$04.03 .01 /$ SR10 $04.03 .01 /$ SR11
$04.02 .03 .01 / \mathrm{SR} 48$

$04.02 .03 .01 /$ SR5 1

$04.02 .04 .01 /$ SR3 8

$04.02 .04 .01 /$ SR 41
$04.02 .03 .01 /$ SR2 8

$04.02 .04 .01 /$ SR15

$04.03 .01 /$ SR06 $04.03 .01 /$ SR0 7 
\#D $3.3 .9:$

$04.02 .03 .01 /$ SR37 $04.02 .03 .02 /$ SR5 $704.02 .04 .01 /$ SR2 9

$04.02 .04 .02 /$ SR2 $404.03 .01 /$ SR0 $64.03 .01 /$ SR0 $04.03 .01 /$ SR 10

$04.03 .01 /$ SR11

\#D 3.3.10:

$04.02 .03 .01 /$ SR4 $304.02 .03 .02 /$ SR61 $04.02 .04 .01 /$ SR3 4

$04.02 .04 .02 /$ SR2 $704.03 .01 /$ SR0 $64.03 .01 /$ SR0 $0404.03 .01 /$ SR10

$04.03 .01 /$ SR11

\#D 3.3.11:

$04.02 .03 .01 /$ SR4 $804.02 .03 .01 /$ SR49 $04.02 .03 .02 /$ SR64

$04.02 .04 .01 /$ SR3 $804.02 .04 .01 /$ SR39 $04.02 .04 .02 /$ SR29 $04.03 .01 /$ SR0 6 $04.03 .01 /$ SR07 $04.03 .01 /$ SR10 $04.03 .01 /$ SRI1

\#D $3.3 .12:$

$02.03 .03 /$ SR15 $04.02 .03 .01 /$ SR03 $04.02 .03 .01 /$ SR11 $04.02 .03 .01 /$ SR5 8 $04.02 .03 .01 /$ SR61 $04.02 .03 .01 /$ SR62 $04.02 .03 .01 /$ SR63

\#D $3.3 .13:$

$02.03 .03 /$ SR15 $04.02 .03 .01 /$ SR11 $04.02 .03 .01 /$ SR80 $04.02 .03 .01 /$ SR 82 $04.02 .03 .01 /$ SR83

\#D 3.3.14:

$02.03 .03 /$ SR15 $04.02 .03 .01 /$ SR06

\#D 3.3.15:

$02.03 .03 /$ SR15

\#D 3.3.16:

$02.03 .03 /$ SR15 $04.02 .03 .01 /$ SR11 $04.02 .03 .01 /$ SR6 $64.02 .03 .01 /$ SR70 $04.02 .03 .01 /$ SR71

\#D 3.3.17:

$02.03 .03 /$ SR15 $04.02 .03 .01 /$ SR11 $04.02 .03 .01 /$ SR74 $04.02 .03 .01 /$ SR77 $04.02 .03 .01 /$ SR78

\#D $3.3 .18:$

$02.03 .03 /$ SR15 $04.02 .03 .01 /$ SR11 $04.02 .03 .01 /$ SR74 $04.02 .03 .01 /$ SR77 $04.02 .03 .01 /$ SR78

\#D 3.4.1:

$04.02 .04 .01 /$ SR05 $04.02 .04 .01 /$ SR48 $04.02 .04 .01 /$ SR5 1

$04.02 .04 .01 /$ SR52 $04.02 .04 .01 /$ SR53 $04.02 .04 .01 /$ SR5 4

\#D $3.4 .2:$

$04.02 .04 .01 /$ SR05 $04.02 .04 .01 /$ SR71 $04.02 .04 .01 /$ SR73

$04.02 .04 .01 /$ SR74

\#D 3.4.5:

$04.02 .04 .01 /$ SR05 $04.02 .04 .01 /$ SR57 $04.02 .04 .01 /$ SR61

$04.02 .04 .01 /$ SR 62 
\#D 3.4.6:

$04.02 .04 .01 /$ SR05 $04.02 .04 .01 /$ SR65 $04.02 .04 .01 /$ SR6 8

$04.02 .04 .01 /$ SR 69

\#D 3.4.7:

$04.02 .04 .01 /$ SR0 $504.02 .04 .01 /$ SR65 $04.02 .04 .01 /$ SR 68 $04.02 .04 .01 /$ SR 69

\#D 3.4.8:

$04.02 .04 .01 /$ SR0 $504.02 .04 .01 /$ SR76 $04.02 .04 .01 /$ SR78

$04.02 .04 .01 /$ SR79 $04.02 .04 .01 /$ SR83 $04.02 .04 .01 /$ SR 84

\#D 3.5:

$04.03 .01 /$ SR08 $04.03 .01 /$ SR09

\#D 3.5.1:

$04.02 .05 .02 /$ SR0 $34.02 .05 .02 /$ SR0 $404.02 .05 .02 /$ SR0 6

$04.02 .05 .02 /$ SR0 $704.02 .05 .02 /$ SR0 $04.02 .05 .02 /$ SR0 9

$04.02 .05 .02 /$ SR10 $04.02 .05 .02 /$ SR3 $504.02 .05 .02 /$ SR 39

$04.02 .05 .02 /$ SR5 1

\#D $3.5 .2:$

$04.02 .05 .01 /$ SR03 $04.02 .05 .01 /$ SR0 $404.02 .05 .01 /$ SR0 7

$04.02 .05 .01 /$ SR08 $04.02 .05 .01 /$ SR09 $04.02 .05 .01 /$ SR 10

$04.02 .05 .01 /$ SR11 $04.02 .05 .01 /$ SR12 $04.02 .05 .01 /$ SR13

$04.02 .05 .01 /$ SR14 $04.02 .05 .01 /$ SR15 $04.02 .05 .01 /$ SR16

$04.02 .05 .01 /$ SR17

\#D 3.5.3:

$04.02 .05 .01 /$ SR03 $04.02 .05 .01 /$ SR0 $404.02 .05 .01 /$ SR 14

$04.02 .05 .01 /$ SR15 $04.02 .05 .01 /$ SR19 $04.02 .05 .01 /$ SR20

$04.02 .05 .01 /$ SR2 $104.02 .05 .01 /$ SR2 $404.02 .05 .01 / S R 25$

\#D 3.5.4:

$04.02 .05 .01 /$ SR03 $04.02 .05 .01 /$ SR0 $404.02 .05 .01 /$ SR 10

$04.02 .05 .01 /$ SR13 $04.02 .05 .01 /$ SR14 $04.02 .05 .01 /$ SR2 7

$04.02 .05 .01 /$ SR2 $84.02 .05 .01 /$ SR2 $94.02 .05 .01 /$ SR 30

$04.02 .05 .01 /$ SR 31

\#D 3.5.5:

$04.02 .05 .01 /$ SR0 8 $04.02 .05 .01 /$ SR09 $04.02 .05 .02 /$ SR35 $04.03 .01 /$ SR0 6 $04.03 .01 /$ SR07 $04.03 .01 /$ SR10 $04.03 .01 /$ SR 11

\#D 3.5.6:

$04.02 .05 .01 /$ SR14 $04.02 .05 .01 /$ SR15 $04.02 .05 .01 /$ SR20

$04.02 .05 .01 /$ SR2 $104.02 .05 .02 /$ SR39 $04.03 .01 /$ SR0 $604.03 .01 /$ SR0 7

$04.03 .01 /$ SR10 $04.03 .01 /$ SRI1

\#D 3.5.7:

$04.02 .05 .01 /$ SR10 $04.02 .05 .01 /$ SR13 $04.02 .05 .01 /$ SR14

$04.02 .05 .01 /$ SR2 $804.02 .05 .01 /$ SR29 $04.02 .05 .02 /$ SR5 $104.03 .01 /$ SR0 6

$04.03 .01 /$ SR0 $704.03 .01 /$ SR10 $04.03 .01 /$ SR 11

\#D $3.6:$ 
$04.03 .01 /$ SR08 $04.03 .01 /$ SR09

\#D 3.6.1:

$04.02 .05 .03 /$ SR03 $04.02 .05 .03 /$ SR0 $504.02 .05 .03 /$ SR0 6

$04.02 .05 .03 /$ SR07 $04.02 .05 .03 /$ SR0 8 $04.02 .05 .03 /$ SR09

$04.02 .05 .03 /$ SR30 $04.02 .05 .03 /$ SR3 $804.02 .05 .03 /$ SR 46

$04.02 .05 .03 /$ SR50

\#D 3.6 .2 :

$04.02 .05 .01 /$ SR0 $404.02 .05 .01 /$ SR34 $04.02 .05 .01 /$ SR3 5

$04.02 .05 .01 /$ SR3 $64.02 .05 .01 /$ SR3 $84.02 .05 .01 /$ SR3 9

\#D 3.6.3:

$04.02 .05 .01 /$ SR0 $404.02 .05 .01 /$ SR4 $104.02 .05 .01 / S R 42$

$04.02 .05 .01 /$ SR4 $304.02 .05 .01 /$ SR4 $704.02 .05 .01 /$ SR 48

\#D $3.6 .4:$

$04.02 .05 .01 /$ SR0 $404.02 .05 .01 /$ SR50 $04.02 .05 .01 /$ SR51

$04.02 .05 .01 /$ SR52 $04.02 .05 .01 /$ SR53 $04.02 .05 .01 /$ SR5 4

\#D $3.6 .5:$

$04.02 .05 .01 /$ SR0 $404.02 .05 .01 /$ SR56 $04.02 .05 .01 /$ SR5 7

$04.02 .05 .01 /$ SR58 $04.02 .05 .01 /$ SR59 $04.02 .05 .01 /$ SR60

\#D 3.6.6:

$04.02 .05 .01 /$ SR35 $04.02 .05 .03 /$ SR30 $04.03 .01 /$ SR0 $604.03 .01 /$ SR0 7

$04.03 .01 /$ SR10 $04.03 .01 /$ SR11

\#D $3.6 .7:$

$04.02 .05 .01 /$ SR42 $04.02 .05 .01 /$ SR43 $04.02 .05 .03 /$ SR3 $04.03 .01 /$ SR0 6 $04.03 .01 /$ SR07 $04.03 .01 /$ SR10 $04.03 .01 /$ SR11

\#D 3.6.8:

$04.02 .05 .01 /$ SR51 $04.02 .05 .01 /$ SR52 $04.02 .05 .03 /$ SR4 $604.03 .01 /$ SR0 6 $04.03 .01 /$ SR07 $04.03 .01 /$ SR10 $04.03 .01 /$ SR11

\#D 3.6.9:

$04.02 .05 .01 /$ SR57 $04.02 .05 .01 /$ SR58 $04.02 .05 .03 /$ SR50 $04.03 .01 /$ SR0 6 $04.03 .01 /$ SR07 $04.03 .01 /$ SR10 $04.03 .01 /$ SRI1

\#D $3.7:$

$04.02 .05 .01 /$ SR 79

\#D 3.7.1:

$04.02 .05 .01 /$ SR03 $04.02 .05 .01 /$ SR04 $04.02 .05 .01 /$ SR62

$04.02 .05 .01 /$ SR63 $04.02 .05 .01 /$ SR64 $04.02 .05 .01 /$ SR65

$04.02 .05 .01 /$ SR71 $04.02 .05 .01 /$ SR73 $04.02 .05 .01 /$ SR74

$04.02 .05 .01 /$ SR76 $04.02 .05 .01 /$ SR7 7

\#D 3.7.2:

$04.02 .05 .01 /$ SR03 $04.02 .05 .01 /$ SR0 $404.02 .05 .01 /$ SR68

$04.02 .05 .01 /$ SR69 $04.02 .05 .01 /$ SR70 $04.02 .05 .01 /$ SR71

$04.02 .05 .01 /$ SR72 $04.02 .05 .01 /$ SR73 $04.02 .05 .01 /$ SR74

$04.02 .05 .01 /$ SR76 $04.02 .05 .01 /$ SR7 $704.02 .05 .01 /$ SR80 
$04.02 .05 .01 /$ SR81

\#D 3.7.3:

$04.02 .05 .01 /$ SR03 $04.02 .05 .01 /$ SR0 $404.02 .05 .01 /$ SR68

$04.02 .05 .01 /$ SR69 $04.02 .05 .01 /$ SR70 $04.02 .05 .01 /$ SR71

$04.02 .05 .01 /$ SR73 $04.02 .05 .01 /$ SR74 $04.02 .05 .01 /$ SR76

$04.02 .05 .01 /$ SR77 $04.02 .05 .01 /$ SR $8204.02 .05 .01 /$ SR 83

\#D $3.9:$

$04.01 /$ SRO1

\#D 3.11:

$02.03 .03 /$ SR0 $62.03 .03 /$ SR18 $02.03 .03 /$ SR19 $02.03 .03 /$ SR20

$04.03 .04 .01 /$ SR11 $04.03 .04 .01 /$ SR12 $04.03 .04 .01 /$ SR13

$04.03 .04 .01 /$ SR14 $04.03 .04 .01 /$ SR15 $04.03 .04 .02 /$ SR0 4

$04.03 .04 .02 /$ SR0 $84.03 .04 .02 /$ SR0 9

\#D $3.12:$

$04.02 .05 .01 /$ SR79

\#D 3.12.1:

$02.03 .03 /$ SR10 04.01/SR01 $06.01 .02 /$ SR15 06.01.02/SR16

$06.01 .02 /$ SR17 06.01.02/SR20 06.01.02/SR21 $06.01 .02 /$ SR22

$06.01 .02 /$ SR 47

\#D $3.12 .2:$

$02.03 .03 /$ SR10 $04.01 /$ SR01 $06.01 .02 /$ SR08 $06.01 .02 /$ SR16

$06.01 .02 /$ SR17 06.01.02/SR20 06.01.02/SR21 $06.01 .02 /$ SR23

$06.01 .02 /$ SR 47

\#D 3.12.3:

$02.03 .03 /$ SR10 $06.01 .02 / S R 4206.01 .02 / S R 4306.01 .02 / S R 44$

$06.01 .02 / S R 4806.01 .02 / S R 49$

\#D 4.1:

05.01.01/SR01 05.01.01/SR04 05.01.01/SR05 05.01.01/SR06

05.01.01/SR07 05.01.01/SR11 05.01.01/SR12

\#D $4.2:$

$02.01 .02 .01 /$ SR05 $02.01 .02 .02 /$ SR01 $02.01 .02 .02 / S R 02$

$02.01 .02 .02 /$ SR05 $02.01 .02 .02 /$ SR0 $02.01 .02 .03 /$ SR0 2

$02.01 .03 .01 /$ SR01 $02.01 .03 .01 /$ SR03 $02.01 .03 .03 /$ SR0 1

$02.01 .03 .03 /$ SR03 $02.02 .01 /$ SR01 $02.02 .01 /$ SR0 $402.02 .01 /$ SR0 5

$02.02 .01 /$ SR0 $02.02 .01 /$ SR09 $02.03 .01 /$ SR01 $02.03 .01 /$ SR02

$02.03 .01 /$ SR03

\#D 4.3:

$02.01 .02 .01 /$ SR0 $02.01 .02 .01 /$ SR07 $02.01 .02 .02 / S R 11$

$02.01 .02 .02 /$ SR12 $02.01 .03 .01 /$ SR0 $402.01 .03 .01 /$ SR0 5

$02.01 .03 .01 /$ SR06 $02.01 .03 .03 /$ SR04 02.01.03.03/SR0 5

$02.01 .03 .03 /$ SR0 $62.02 .01 /$ SR02 $02.02 .01 /$ SR03 $02.02 .01 /$ SR0 8

$02.02 .03 /$ SR02 $02.02 .03 /$ SR03 $02.02 .03 /$ SR0 $402.02 .03 /$ SR0 6

$02.02 .03 /$ SR0 $02.02 .03 /$ SR08 $02.02 .03 /$ SR09 $02.02 .03 /$ SR10

$02.02 .04 /$ SR01 $02.02 .04 /$ SR02 $02.02 .04 /$ SR0 $62.02 .04 /$ SR0 7 


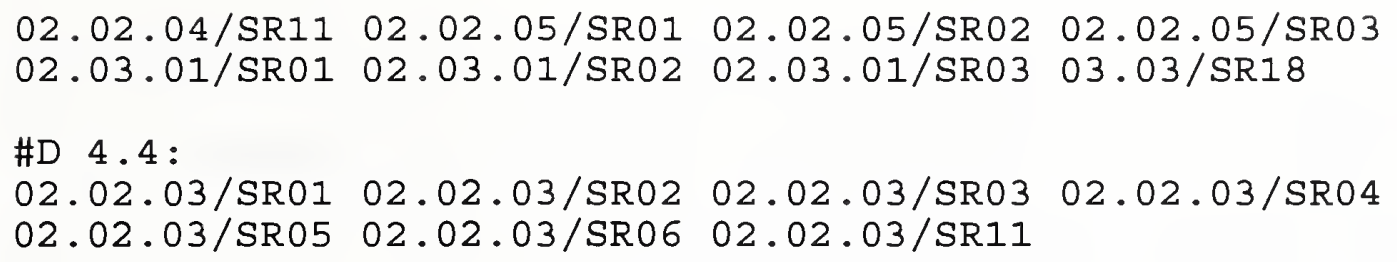


DATA STRUCTURE CROSS-REFERENCE

$06.02 .02 /$ SR29

\#D $5.4 .2 .3:$

$04.02 .05 .01 /$ SR79 $06.02 .02 /$ SR17 $06.02 .02 / S R 2506.02 .02 / S R 26$

\#D 5.4.2.3.1:

$06.02 .01 /$ SR01 $06.02 .01 /$ SR02

\#D $5.4 .2 .3 .2:$

$06.02 .01 /$ SR01 $06.02 .01 /$ SR02

\#D $5.4 .2 .4:$

$06.02 .02 /$ SR17 $06.02 .02 /$ SR2 $506.02 .02 /$ SR2 6

\#D 5.4.2.4.1:

$06.02 .01 /$ SR01 $06.02 .01 /$ SR02 $06.02 .02 /$ SR14 $06.02 .02 /$ SR15

$06.02 .02 /$ SR16 $06.02 .02 /$ SR 23

\#D 5.4.2.4.2:

$06.02 .01 /$ SR01 $06.02 .01 /$ SR02 $06.02 .02 /$ SR14 $06.02 .02 /$ SR15

$06.02 .02 /$ SR16 $06.02 .02 /$ SR23

\#D $5.4 .2 .4 .3:$

$06.02 .02 /$ SR14 $06.02 .02 /$ SR15 $06.02 .02 /$ SR16 $06.02 .02 /$ SR2 3

\#D 5.4.2.4.4:

$06.02 .02 /$ SR14 $06.02 .02 /$ SR15 $06.02 .02 /$ SR23

\#D 5.4.2.4.5:

$06.02 .02 /$ SR14 $06.02 .02 /$ SR15 $06.02 .02 /$ SR16 $06.02 .02 /$ SR23

\#D $5.4 .2 .4 .6:$

$06.02 .02 /$ SR14 $06.02 .02 /$ SR15 $06.02 .02 /$ SR16 $06.02 .02 /$ SR2 3

\#D $5.5:$

$04.03 .03 /$ SR0 5

\#D 5.5.1:

$04.03 .03 /$ SR06 04.03.03/SR07 04.03.03/SR12 $04.03 .03 /$ SR15

\#D 5.5.2:

$04.03 .03 /$ SR0 $64.03 .03 /$ SR0 $04.03 .03 /$ SR13

\#D $5.5 .3:$

$04.03 .03 /$ SR0 $404.03 .03 /$ SR14

\#D $5.6:$

$04.01 /$ SR01 05.02/SR10 06.03/SR05 06.03/SR16 06.03/SRI7

\#D 5.6.1:

$06.03 /$ SR15 $06.03 /$ SR18 $06.03 /$ SR19 $06.03 /$ SR20

\#D 5.6.2:

06.03/SR01 06.03/SR06 06.03/SR07 06.03/SR08 06.03/SR12 06.03/SR13 
DATA STRUCTURE CROSS-REFERENCE

$06.03 /$ SR14

\#D $5.6 .3:$

$04.02 .05 .01 /$ SR79 $06.03 /$ SR01 $06.03 /$ SR08 $06.03 /$ SR12 $06.03 /$ SR 13

\#D $5.6 .4:$

$06.03 /$ SR01 06.03/SR09 06.03/SR10 06.03/SR11 06.03/SR12 06.03/SR13 $06.03 /$ SR14

\#D 5.6.5:

$04.02 .05 .01 /$ SR79 $06.03 /$ SR01 $06.03 /$ SR11 $06.03 /$ SR $1206.03 /$ SR 13

\#D 5.7:

$02.01 .02 .01 /$ SR0 $402.01 .02 .02 /$ SR10 $02.01 .03 .01 /$ SR01

$02.01 .03 .01 /$ SR03 $02.01 .03 .02 /$ SR0 $502.01 .03 .02 /$ SR0 6

$02.01 .03 .03 /$ SR01 $02.01 .03 .03 /$ SR0 $305.02 /$ SR10 $05.03 /$ SR0 6

$05.03 /$ SR0 $85.03 /$ SR09 $05.03 /$ SR10 $05.03 /$ SR13

\#D 5.7.1:

$05.03 /$ SR02 $05.03 /$ SR0 $505.03 /$ SR14

\#D $5.7 .2:$

$05.03 /$ SR03 $05.03 /$ SR0 $505.03 /$ SR14

\#D 5.8.1:

$04.03 .03 /$ SR0 $04.03 .03 /$ SR0 $05.02 / S R 01 \quad 05.02 / S R 03 \quad 05.02 / S R 04$

05.02 /SR0 $85.02 /$ SR2 $205.02 /$ SR2 $305.02 /$ SR2 $405.02 /$ SR2 $505.02 / S R 26$

$05.02 /$ SR2 $705.02 /$ SR2 $805.02 /$ SR29 $05.02 /$ SR31 $05.02 /$ SR 32

\#D $5.8 .2:$

$04.03 .03 /$ SR0 $65.02 /$ SR02 05.02/SR03 05.02/SR04 05.02/SR0 8

$05.02 /$ SR2 $305.02 /$ SR2 $405.02 /$ SR2 $505.02 /$ SR2 $605.02 /$ SR2 $705.02 / S R 28$

$05.02 /$ SR2 $905.02 /$ SR3 $105.02 /$ SR3 2

\#D $5.8 .3:$

$04.03 .03 /$ SR06 $04.03 .03 /$ SR0 $05.02 /$ SR04 05.02/SR09 $05.02 /$ SR 11

$05.02 /$ SR12

\#D $5.8 .4:$

05.02/SR04 05.02/SR09 $05.02 /$ SR11 $05.02 /$ SR12 $05.02 /$ SR13 $05.02 /$ SR14

$05.02 /$ SR15 05.02/SR16 05.02/SR17 05.02/SR18 05.02/SR22 $05.02 / S R 23$

$05.02 /$ SR2 $405.02 / S R 2505.02 / S R 26 \quad 05.02 / S R 2705.02 / S R 2805.02 / S R 29$

$05.02 /$ SR $3105.02 /$ SR32 $06.02 .02 / S R 2706.02 .02 /$ SR2 $806.03 /$ SR1 8

$06.03 /$ SR19

\#D $5.9:$

$04.02 .01 .02 /$ SR18

\#D $5.9 .1:$

$04.02 .01 .02 /$ SR0 $04.02 .01 .02 /$ SR0 $04.02 .01 .02 /$ SR 10

$04.02 .01 .02 /$ SR12 $04.02 .01 .02 /$ SR13

\#D $5.9 .2:$

$04.02 .01 .02 /$ SR06 $04.02 .01 .02 /$ SR0 $04.02 .01 .02 /$ SR 11 
$04.02 .01 .02 /$ SR14 $04.02 .01 .02 /$ SR15 $04.02 .01 .02 /$ SR16 $04.02 .01 .02 / S R 2404.02 .01 .02 / S R 25$

\#D $5.9 .2 .1:$

$04.02 .01 .02 /$ SR10

$04.02 .01 .02 /$ SR3 5

$04.02 .01 .02 /$ SR 45

$04.02 .01 .02 /$ SR 49

\#D 5.9.2.2:

$04.02 .01 .02 /$ SR3 $34.02 .01 .02 /$ SR3 $04.02 .01 .02 /$ SR 35

\#D $5.9 .2 .3:$

$04.02 .01 .02 /$ SR $4104.02 .01 .02 /$ SR $4204.02 .01 .02 / S R 43$

$04.02 .01 .02 /$ SR $4404.02 .01 .02 /$ SR 45

\#D 5.9.2.4:

$04.02 .01 .02 /$ SR4 $604.02 .01 .02 / S R 4704.02 .01 .02 / S R 48$

$04.02 .01 .02 /$ SR 49

\#D $5.10:$

$04.02 .02 .02 /$ SR 18

\#D 5.10.1:

$04.02 .02 .02 /$ SR0 $64.02 .02 .02 /$ SR0 $04.02 .02 .02 /$ SR 10

$04.02 .02 .02 /$ SR12 $04.02 .02 .02 /$ SR13

\#D $5.10 .2:$

$04.02 .02 .02 /$ SR0 $64.02 .02 .02 /$ SR0 7

$04.02 .02 .02 /$ SR14 $04.02 .02 .02 /$ SR15

$04.02 .02 .02 /$ SR2 $404.02 .02 .02 /$ SR2 5

\#D 5.10.2.1:

$04.02 .02 .02 /$ SR 10

$04.02 .02 .02 /$ SR3 5

$04.02 .02 .02 /$ SR 45

$04.02 .02 .02 /$ SR50

\#D $5.10 .2 .2:$

$04.02 .02 .02 /$ SR3 $304.02 .02 .02 /$ SR3 $404.02 .02 .02 /$ SR 35

\#D $5.10 .2 .3:$

$04.02 .02 .02 / S R 41 \quad 04.02 .02 .02 / S R 42 \quad 04.02 .02 .02 / S R 43$

$04.02 .02 .02 /$ SR $4404.02 .02 .02 /$ SR 45

\#D $5.10 .2 .4:$

$04.02 .02 .02 /$ SR $4704.02 .02 .02 / S R 48 \quad 04.02 .02 .02 / S R 49$

$04.02 .02 .02 /$ SR50

\#D 5.11:

$04.02 .03 .02 /$ SR18

\#D 5.11.1:

$04.02 .03 .02 /$ SR0 $04.02 .03 .02 /$ SR0 $04.02 .03 .02 /$ SR 10 
$04.02 .03 .02 /$ SR12 $04.02 .03 .02 /$ SR13 $04.02 .04 .02 /$ SR0 4 $04.02 .04 .02 /$ SR0 5

\#D $5.11 .2:$

$04.02 .03 .02 /$ SR0 $64.02 .03 .02 /$ SR0 $704.02 .03 .02 /$ SR11

$04.02 .03 .02 /$ SR14 $04.02 .03 .02 /$ SR15 $04.02 .03 .02 /$ SR16

$04.02 .03 .02 / S R 2404.02 .03 .02 / S R 2504.02 .04 .02 / S R 04$

$04.02 .04 .02 /$ SR0 $504.02 .04 .02 /$ SR09

\#D $5.11 .2 .1:$

$04.02 .03 .02 /$ SR10 $04.02 .03 .02 /$ SR12 $04.02 .03 .02 /$ SR13

$04.02 .03 .02 /$ SR3 $804.02 .03 .02 /$ SR39 $04.02 .03 .02 /$ SR 57

$04.02 .03 .02 /$ SR61 $04.02 .03 .02 /$ SR $6404.02 .04 .02 / S R 11$

$04.02 .04 .02 /$ SR12 $04.02 .04 .02 / S R 2404.02 .04 .02 / S R 27$

$04.02 .04 .02 /$ SR2 9

\#D $5.11 .2 .2:$

$04.02 .03 .02 /$ SR3 $54.02 .03 .02 /$ SR3 $64.02 .03 .02 /$ SR3 8

$04.02 .03 .02 /$ SR $4004.02 .04 .02 /$ SR11 $04.02 .04 .02 /$ SR 13

\#D $5.11 .2 .3:$

$04.02 .03 .02 /$ SR3 $204.02 .03 .02 /$ SR3 $304.02 .03 .02 /$ SR3 4

$04.02 .03 .02 /$ SR3 $504.02 .03 .02 /$ SR3 $604.02 .03 .02 /$ SR3 9

$04.02 .03 .02 /$ SR4 $704.02 .04 .02 /$ SR10 $04.02 .04 .02 /$ SR12

$04.02 .04 .02 /$ SR2 0

\#D $5.11 .2 .4:$

$04.02 .03 .02 /$ SR5 $604.02 .03 .02 /$ SR5 $704.02 .03 .02 /$ SR59

$04.02 .04 .02 / S R 2404.02 .04 .02 / S R 26$

\#D $5.11 .2 .5:$

$04.02 .03 .02 /$ SR60 $04.02 .03 .02 /$ SR61 $04.02 .04 .02 /$ SR2 7

\#D $5.11 .2 .6:$

$04.02 .03 .02 /$ SR63 $04.02 .03 .02 /$ SR64 $04.02 .03 .02 /$ SR 65

$04.02 .03 .02 /$ SR6 $64.02 .04 .02 /$ SR29 $04.02 .04 .02 /$ SR30

$04.02 .04 .02 / S R 31$

\#D $5.12:$

$04.02 .05 .02 /$ SR 19

\#D 5.12.1:

$04.02 .05 .02 /$ SR0 $704.02 .05 .02 /$ SR0 $04.02 .05 .02 /$ SR11 $04.02 .05 .02 /$ SR13 $04.02 .05 .02 /$ SR14

\#D $5.12 .2:$

$04.02 .05 .02 /$ SR07 $04.02 .05 .02 /$ SR08 $04.02 .05 .02 /$ SR 12

$04.02 .05 .02 /$ SR15 $04.02 .05 .02 /$ SR16 $04.02 .05 .02 /$ SR1 7

$04.02 .05 .02 /$ SR2 $504.02 .05 .02 /$ SR2 6

\#D $5.12 .2 .1:$

$04.02 .05 .02 /$ SRI1 $04.02 .05 .02 /$ SR13 $04.02 .05 .02 /$ SR14 $04.02 .05 .02 /$ SR35 $04.02 .05 .02 /$ SR39 $04.02 .05 .02 /$ SR51 


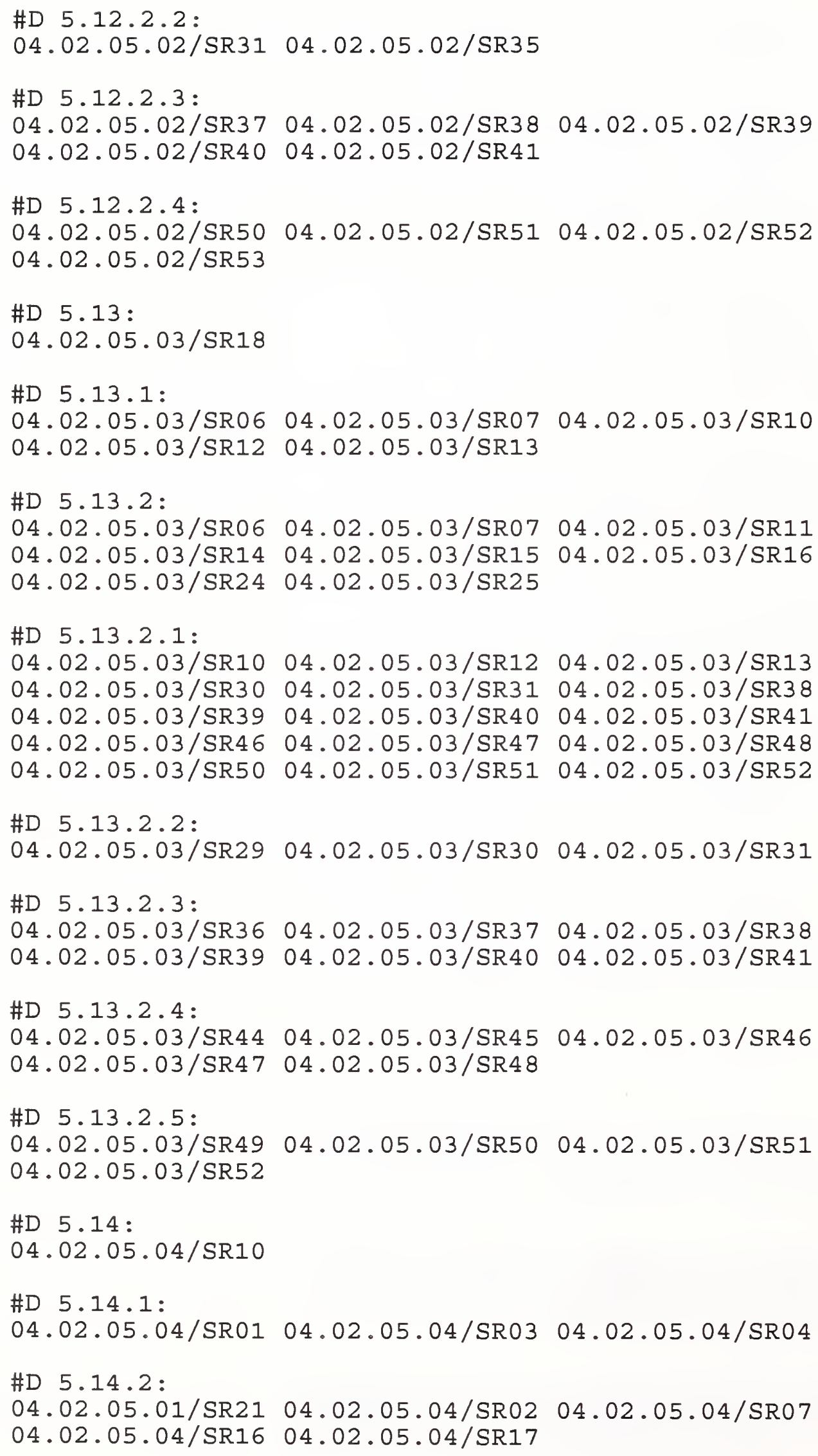


\#D 5.14.2.1:

$04.02 .05 .01 /$ SR2 0

$04.02 .05 .02 / S R 40$

$04.02 .05 .02 / S R 41$

$04.02 .05 .04 /$ SR01

$04.02 .05 .04 /$ SR03

$04.02 .05 .04 /$ SR0 4

$04.02 .05 .04 /$ SR0 7

\#D 5.14.2.2:

$04.02 .05 .01 /$ SR75

$04.02 .05 .01 /$ SR79

$04.02 .05 .01 /$ SR76

$04.02 .05 .01 /$ SR77

$04.02 .05 .04 /$ SR19

$04.02 .05 .04 /$ SR0 5

$04.02 .05 .04 /$ SR0 6

$04.02 .05 .04 / S R 20$

$04.02 .05 .04 /$ SR2 1

\#D 5.15.1:

$04.03 .02 .01 /$ SR02 $04.03 .02 .01 /$ SR0 5

$04.03 .02 .01 /$ SR09

$04.03 .02 .01 /$ SR10

$04.03 .02 .02 /$ SR17

$04.03 .02 .02 / S R 2104.03 .02 .02 / S R 27$

$04.03 .02 .02 / \mathrm{SR} 20$

\#D 5.15 .2 :

$04.03 .02 .01 /$ SR01 $04.03 .02 .01 /$ SR03

$04.03 .02 .01 /$ SR0 $04.03 .02 .01 /$ SR0 8

$04.03 .02 .01 / S R 04$

\#D $5.15 .3:$

$04.02 .01 .02 /$ SR4 $804.02 .01 .02 /$ SR49

$04.02 .02 .02 /$ SR 49

$04.02 .02 .02 /$ SR 50

$04.02 .03 .02 /$ SR 65

$04.02 .04 .02 / S R 31$

$04.02 .03 .02 /$ SR 66

$04.02 .04 .02 /$ SR 30

$04.02 .05 .02 /$ SR 53

$04.02 .05 .03 /$ SR 51

$04.02 .05 .02 /$ SR 52

$04.02 .05 .04 /$ SR2 0

$04.02 .05 .04 /$ SR21

$04.02 .05 .03 /$ SR 52

$04.03 .02 .02 /$ SR14 $04.03 .02 .02 /$ SR33

$04.03 .02 .02 /$ SR 13

\#D $5.15 \cdot 3.1:$

$04.02 .01 .01 /$ SR21

$04.02 .02 .01 /$ SR2 3

$04.02 .01 .01 /$ SR22

$04.02 .02 .01 /$ SR2 2

$04.02 .03 .01 / S R 48$

$04.02 .03 .01 /$ SR 49

$04.02 .04 .01 /$ SR3 8

$04.02 .04 .01 /$ SR39

$04.02 .05 .01 /$ SR2 8

$04.02 .05 .01 /$ SR29

$04.02 .05 .01 /$ SR5 7

$04.02 .05 .01 /$ SR5 8

$04.03 .02 .01 /$ SR01

$04.03 .02 .01 /$ SR02

$04.03 .02 .01 /$ SR0 3

$04.03 .02 .01 /$ SR0 4

$04.03 .02 .01 /$ SR0 5

$04.03 .02 .01 /$ SR0 6

$04.03 .02 .02 /$ SR 30

$04.03 .02 .02 /$ SR3 1

$04.03 .02 .02 /$ SR 32

\#D $5.15 \cdot 3 \cdot 2:$

$04.02 .01 .01 /$ SR21

$04.02 .02 .01 /$ SR2 3

$04.02 .01 .01 /$ SR22

$04.02 .02 .01 / \mathrm{SR} 22$

$04.02 .03 .01 /$ SR4 $804.02 .03 .01 /$ SR49

$04.02 .04 .01 /$ SR3 8

$04.02 .04 .01 /$ SR39

$04.02 .05 .01 /$ SR2 8

$04.02 .05 .01 /$ SR29

$04.02 .05 .01 /$ SR5 7

$04.02 .05 .01 /$ SR 58

$04.03 .02 .01 /$ SR02

$04.03 .02 .01 /$ SR0 4

$04.03 .02 .01 /$ SR0 5

$04.03 .02 .01 /$ SR0 6

$04.03 .02 .02 /$ SR2 1

$04.03 .02 .02 /$ SR 26

$04.03 .02 .02 /$ SR2 7

$04.03 .02 .02 /$ SR 30

$04.03 .02 .02 /$ SR 31

$04.03 .02 .02 /$ SR 32

\section{\#D 5.16 :}

$04.03 .04 .02 /$ SR01 $04.03 .04 .02 /$ SR02 $04.03 .04 .02 /$ SR0 3

$04.03 .04 .02 /$ SR0 $404.03 .04 .02 /$ SR10 $04.03 .04 .02 /$ SR 11

$04.03 .04 .02 /$ SR 12

\#D $5.17:$

$04.03 .04 .02 /$ SR05 $04.03 .04 .02 /$ SR06 04.03.04.02/SR0 


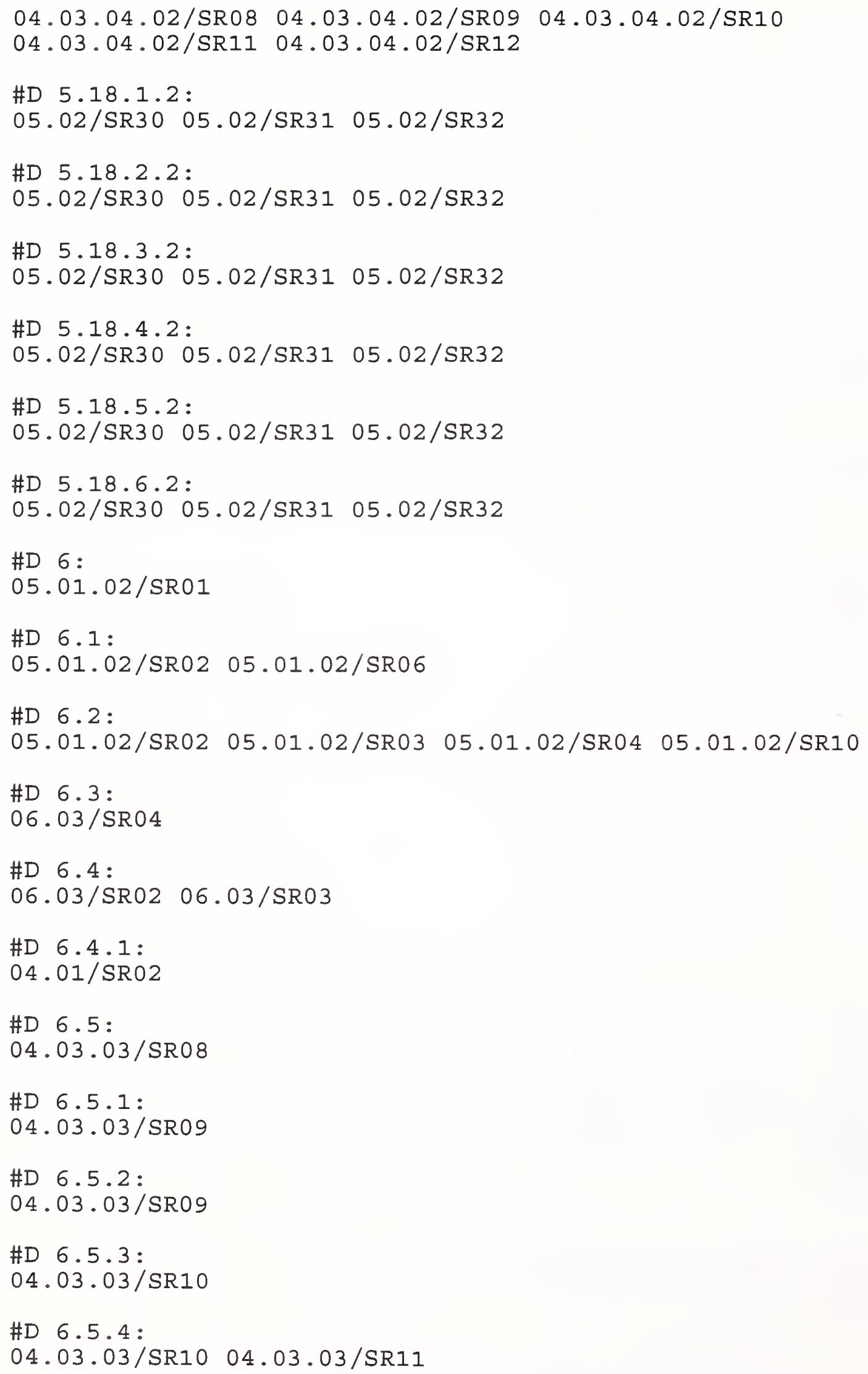




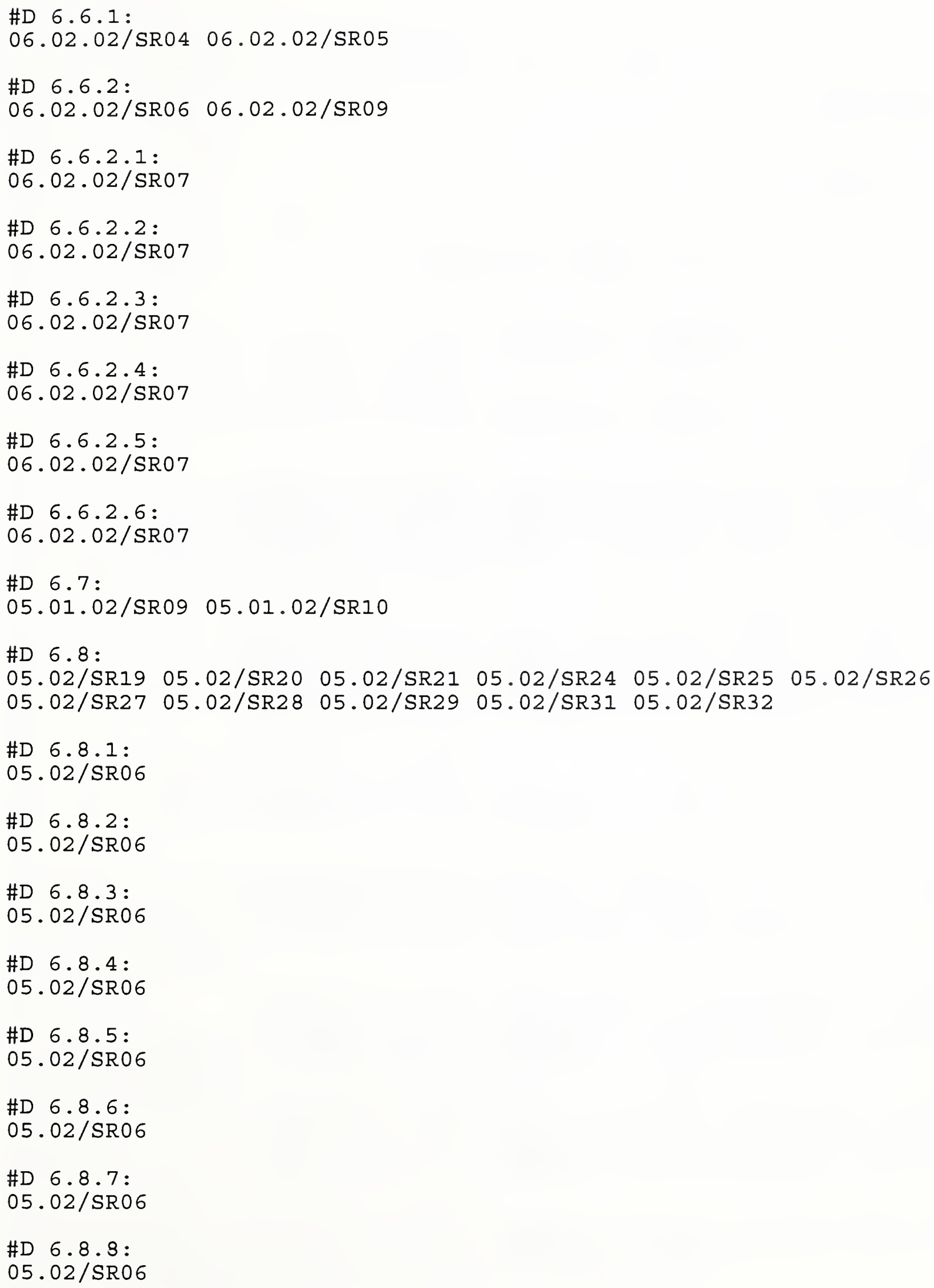




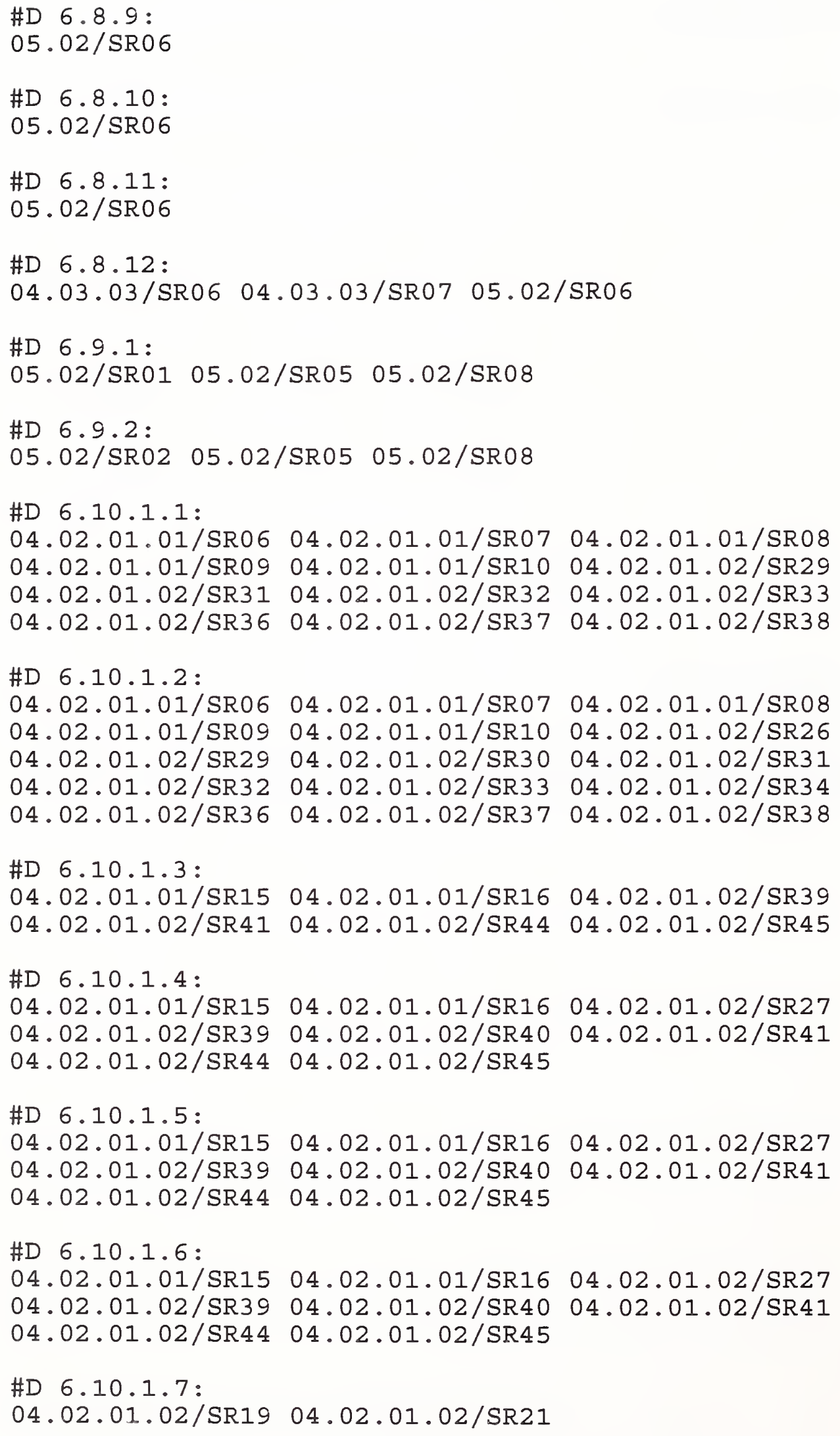


\#D 6.10.2:

$04.02 .01 .02 / S R 20$

$04.02 .01 .02 / \mathrm{SR} 23$

$04.02 .01 .02 /$ SR21 $04.02 .01 .02 /$ SR2 2

$04.02 .01 .02 /$ SR24 $04.02 .01 .02 /$ SR2 5

\#D $6.10 .2 .1:$

$04.02 .01 .02 /$ SR2 6

\#D 6.10.2.2:

$04.02 .01 .02 /$ SR2 7

\#D 6.10.2.3:

$04.02 .01 .02 / S R 28$

\#D 6.11.1.1:

$04.02 .02 .01 / \mathrm{SR} 06$

$04.02 .02 .01 / \mathrm{SR} 09$

$04.02 .02 .02 / \mathrm{SR} 31$

$04.02 .02 .02 / \mathrm{SR} 36$

$04.02 .02 .01 / \operatorname{SR} 0704.02 .02 .01 / \mathrm{SR} 08$

$04.02 .02 .01 /$ SR10 $04.02 .02 .02 /$ SR29

$04.02 .02 .02 / \mathrm{SR} 3204.02 .02 .02 / \mathrm{SR} 33$

$04.02 .02 .02 / \operatorname{SR} 3704.02 .02 .02 /$ SR3 8

\#D 6.11.1.2:

$04.02 .02 .01 /$ SR0 $64.02 .02 .01 /$ SR0 $0404.02 .02 .01 /$ SR0 8

$04.02 .02 .01 /$ SR09 $04.02 .02 .01 /$ SR10 $04.02 .02 .02 / S R 26$

$04.02 .02 .02 /$ SR29 $04.02 .02 .02 /$ SR30 $04.02 .02 .02 / S R 31$

$04.02 .02 .02 / S R 3204.02 .02 .02 / S R 3304.02 .02 .02 / S R 34$

$04.02 .02 .02 /$ SR3 $604.02 .02 .02 /$ SR $3704.02 .02 .02 / S R 38$

\#D 6.11.1.3:

$04.02 .02 .01 /$ SR15 $04.02 .02 .01 /$ SR16 $04.02 .02 .02 /$ SR39

$04.02 .02 .02 /$ SR $4104.02 .02 .02 /$ SR $44 \quad 04.02 .02 .02 / S R 45$

\#D 6.11.1.4:

$04.02 .02 .01 /$ SR15 $04.02 .02 .01 /$ SR16 $04.02 .02 .01 /$ SR17

$04.02 .02 .02 /$ SR2 $704.02 .02 .02 /$ SR39 $04.02 .02 .02 / S R 40$

$04.02 .02 .02 /$ SR41 $04.02 .02 .02 / S R 44 \quad 04.02 .02 .02 / S R 45$

$04.02 .02 .02 / \operatorname{SR} 46$

\#D 6.11.1.5:

$04.02 .02 .01 /$ SR15 $04.02 .02 .01 /$ SR16 $04.02 .02 .01 /$ SR17

$04.02 .02 .02 / S R 2704.02 .02 .02 / S R 3904.02 .02 .02 / S R 40$

$04.02 .02 .02 / S R 4104.02 .02 .02 / S R 44 \quad 04.02 .02 .02 / S R 45$

$04.02 .02 .02 / \mathrm{SR} 46$

\#D 6.11.1.6:

$04.02 .02 .01 /$ SR15 $04.02 .02 .01 /$ SR16 $04.02 .02 .01 /$ SR 17

$04.02 .02 .02 /$ SR2 $704.02 .02 .02 /$ SR39 $04.02 .02 .02 / S R 40$

$04.02 .02 .02 /$ SR4 $104.02 .02 .02 / S R 44 \quad 04.02 .02 .02 / S R 45$

$04.02 .02 .02 / \operatorname{SR} 46$

\#D 6.11.1.7:

$04.02 .02 .02 /$ SR19 $04.02 .02 .02 /$ SR2 1

\#D $6.11 .2:$

$04.02 .02 .02 /$ SR20 $04.02 .02 .02 / S R 2104.02 .02 .02 / S R 22$

$04.02 .02 .02 /$ SR2 $304.02 .02 .02 / S R 2404.02 .02 .02 / S R 25$ 
\#D 6.11.2.1:

$04.02 .02 .02 /$ SR2 6

\#D 6.11.2.2:

$04.02 .02 .02 /$ SR 27

\#D 6.11.2.3:

$04.02 .02 .02 /$ SR2 8

\#D 6.12.1.1:

$04.02 .03 .01 /$ SR19

$04.02 .03 .01 /$ SR2 8

$04.02 .03 .02 /$ SR 47

$04.02 .04 .01 /$ SR14

$04.02 .04 .02 /$ SR13

$04.02 .03 .01 /$ SR21

$04.02 .03 .02 / S R 26$

$04.02 .03 .01 /$ SR22

$04.02 .03 .03 /$ SR01

$04.02 .03 .02 / \mathrm{SR} 40$

$04.02 .04 .01 /$ SR15

$04.02 .04 .01 /$ SR12

$04.02 .04 .02 / S R 20$

\#D 6.12.1.2:

$04.02 .03 .01 /$ SR19

$04.02 .03 .01 /$ SR2 8

$04.02 .03 .02 /$ SR 32

$04.02 .03 .02 / S R 40$

$04.02 .04 .01 /$ SR 12

$04.02 .04 .01 /$ SR2 0

$04.02 .03 .01 / S R 21$

$04.02 .03 .02 /$ SR2 6

$04.02 .03 .02 /$ SR 33

$04.02 .03 .02 /$ SR 47

$04.02 .04 .01 /$ SR14

$04.02 .04 .01 / S R 20$

\#D 6.12.1.2.1:

$04.02 .03 .02 /$ SR31

$04.02 .03 .02 /$ SR 37

$04.02 .04 .02 /$ SRI3

$04.02 .03 .01 /$ SR22

$04.02 .03 .02 /$ SR3 0

$04.02 .03 .02 /$ SR 35

$04.02 .03 .03 /$ SRO1

$04.02 .04 .01 /$ SR15

$04.02 .04 .02 / S R 20$

$04.02 .03 .03 /$ SR1 6

$04.02 .03 .02 /$ SR3 4

$04.02 .03 .02 / S R 36$

$04.02 .03 .03 /$ SR09 $04.02 .03 .03 /$ SR15

\#D 6.12.1.3:

$04.02 .03 .01 / \operatorname{sR2} 9(10)$

$04.02 .04 .02 /$ SRI0

\#D $6.12 .1 .4:$

$04.02 .03 .01 /$ SR29 $04.02 .03 .01 /$ SR30 $04.02 .03 .01 /$ SR61

\#D 6.12.1.5:

$04.02 .03 .01 /$ SR29 $04.02 .03 .01 /$ SR30 $04.02 .03 .01 /$ SR61

\#D 6.12.1.6:

$04.02 .03 .01 /$ SR29 $04.02 .03 .01 /$ SR30 $04.02 .03 .01 /$ SR3 7

$04.02 .03 .01 /$ SR53 $04.02 .03 .01 /$ SR55 $04.02 .03 .01 /$ SR5 7

$04.02 .03 .02 / S R 2704.02 .03 .02 / S R 4804.02 .03 .02 / S R 49$

$04.02 .03 .02 /$ SR51 $04.02 .03 .02 /$ SR53 $04.02 .03 .02 /$ SR5 5

$04.02 .03 .02 /$ SR59 $04.02 .04 .01 / S R 2104.02 .04 .01 / S R 29$

$04.02 .04 .02 /$ SR21 $04.02 .04 .02 /$ SR22 $04.02 .04 .02 /$ SR2 6

\#D 6.12.1.7:

$04.02 .03 .01 /$ SR29 $04.02 .03 .01 /$ SR30 $04.02 .03 .01 /$ SR 37

$04.02 .03 .01 /$ SR53 $04.02 .03 .01 /$ SR54 $04.02 .03 .01 /$ SR5 6

$04.02 .03 .02 /$ SR2 $704.02 .03 .02 /$ SR $4804.02 .03 .02 /$ SR 49

$04.02 .03 .02 /$ SR51 $04.02 .03 .02 /$ SR52 $04.02 .03 .02 /$ SR5 4

$04.02 .03 .02 /$ SR59 $04.02 .04 .01 /$ SR21 $04.02 .04 .01 / S R 29$

$04.02 .04 .02 /$ SR21 $04.02 .04 .02 /$ SR22 $04.02 .04 .02 /$ SR2 6 
\#D $6.12 .1 .8:$

$04.02 .03 .01 /$ SR29 $04.02 .03 .01 /$ SR30 $04.02 .03 .01 /$ SR3 7

$04.02 .03 .01 /$ SR53 $04.02 .03 .01 /$ SR54 $04.02 .03 .01 /$ SR5 6

$04.02 .03 .02 /$ SR27 $04.02 .03 .02 /$ SR4 $804.02 .03 .02 / S R 49$

$04.02 .03 .02 /$ SR51 $04.02 .03 .02 /$ SR52 $04.02 .03 .02 /$ SR5 4

$04.02 .03 .02 /$ SR59 $04.02 .04 .01 /$ SR21 $04.02 .04 .01 /$ SR2 9

$04.02 .04 .02 /$ SR21 $04.02 .04 .02 /$ SR22 $04.02 .04 .02 / S R 26$

\#D 6.12.1.9:

$04.02 .03 .02 /$ SR19 $04.02 .03 .02 /$ SR21

\#D $6.12 .2:$

$04.02 .03 .02 / \mathrm{SR} 2004.02 .03 .02 / \mathrm{SR} 21 \quad 04.02 .03 .02 / \mathrm{SR} 22$

$04.02 .03 .02 /$ SR2 $304.02 .03 .02 / S R 2404.02 .03 .02 / S R 25$

$04.02 .04 .02 /$ SR0 $84.02 .04 .02 /$ SR09

\#D 6.12.2.1:

$04.02 .03 .02 /$ SR2 6

\#D $6.12 .2 .2:$

$04.02 .03 .02 /$ SR2 $604.02 .03 .02 /$ SR2 $704.02 .03 .02 /$ SR2 8

$04.02 .03 .02 /$ SR $3404.02 .04 .02 /$ SR 10

\#D $6.12 .2 .3:$

$04.02 .03 .02 /$ SR2 7

\#D $6.12 .2 .4:$

$04.02 .03 .02 /$ SR2 8

\#D $6.12 .2 .5:$

$04.02 .03 .02 /$ SR2 9

\#D 6.13.1.1:

$04.02 .04 .01 / S R 2104.02 .04 .01 / S R 43 \quad 04.02 .04 .01 / S R 45$

$04.02 .04 .01 /$ SR $4704.02 .04 .01 /$ SR52

\#D $6.13 .1 .2:$

$04.02 .04 .01 /$ SR21 $04.02 .04 .01 /$ SR43 $04.02 .04 .01 / S R 44$

$04.02 .04 .01 /$ SR4 $604.02 .04 .01 /$ SR52

\#D $6.13 .1 .3:$

$04.02 .04 .01 /$ SR21 $04.02 .04 .01 /$ SR43 $04.02 .04 .01 / S R 44$

$04.02 .04 .01 /$ SR $4604.02 .04 .01 /$ SR52

\#D $6.13 .1 .4:$

$04.02 .04 .01 /$ SR77 $04.02 .04 .01 /$ SR78 $04.02 .04 .01 /$ SR79

$04.02 .04 .01 /$ SR81 $04.02 .04 .01 /$ SR82

\#D $6.13 .1 .5:$

$04.02 .04 .01 /$ SR77 $04.02 .04 .01 /$ SR78 $04.02 .04 .01 /$ SR79

$04.02 .04 .01 /$ SR81 $04.02 .04 .01 /$ SR82

\#D 6.14.1.1: 
$04.02 .05 .01 /$ SR06 $04.02 .05 .01 /$ SR08 $04.02 .05 .01 /$ SR09 $04.02 .05 .02 /$ SR 36

\#D 6.14 .1 .2 :

$04.02 .05 .01 /$ SR06 $04.02 .05 .01 /$ SR0 $804.02 .05 .01 /$ SR09

$04.02 .05 .02 /$ SR2 $704.02 .05 .02 /$ SR31 $04.02 .05 .02 /$ SR 32

$04.02 .05 .02 /$ SR $3404.02 .05 .02 /$ SR36 $04.02 .05 .02 /$ SR 42

$04.02 .05 .02 /$ SR $4304.02 .05 .04 /$ SR09 $04.02 .05 .04 /$ SR 14

\#D 6.14.1.2.1:

$04.02 .05 .02 /$ SR33

\#D $6.14 .1 .3:$

$04.02 .05 .01 /$ SR20 $04.02 .05 .01 /$ SR21 $04.02 .05 .01 /$ SR2 2

$04.02 .05 .01 /$ SR2 $304.02 .05 .02 / S R 4204.02 .05 .02 / S R 43$

$04.02 .05 .02 /$ SR $4404.02 .05 .02 /$ SR $4504.02 .05 .02 /$ SR 46

\#D $6.14 .1 .4:$

$04.02 .05 .01 /$ SR20 $04.02 .05 .01 /$ SR21 $04.02 .05 .01 /$ SR2 2

$04.02 .05 .01 /$ SR23 $04.02 .05 .02 /$ SR29 $04.02 .05 .02 /$ SR4 0

$04.02 .05 .02 /$ SR4 $104.02 .05 .02 /$ SR $44 \quad 04.02 .05 .02 / S R 45$

$04.02 .05 .02 /$ SR $4604.02 .05 .02 /$ SR $4704.02 .05 .02 /$ SR 48

$04.02 .05 .02 /$ SR 49

\#D $6.14 .1 .5:$

$04.02 .05 .02 / S R 2004.02 .05 .02 / S R 22$

\#D 6.14.2:

$04.02 .05 .02 /$ SR2 $104.02 .05 .02 /$ SR2 $204.02 .05 .02 /$ SR2 3

$04.02 .05 .02 /$ SR2 $404.02 .05 .02 /$ SR2 $504.02 .05 .02 /$ SR2 6

\#D 6.14.2.1:

$04.02 .05 .02 /$ SR2 $704.02 .05 .02 /$ SR2 $8 \quad 04.02 .05 .02 /$ SR2 9 $04.02 .05 .02 /$ SR 31

\#D 6.14 .2 .2 :

$04.02 .05 .02 /$ SR2 $804.02 .05 .02 /$ SR29

\#D $6.14 .2 .3:$

$04.02 .05 .02 /$ SR30

\#D 6.15.1.1:

$04.02 .05 .01 /$ SR4 $204.02 .05 .01 /$ SR4 $304.02 .05 .01 / S R 44$

$04.02 .05 .01 /$ SR4 $504.02 .05 .01 /$ SR4 $604.02 .05 .03 /$ SR32

$04.02 .05 .03 /$ SR3 $404.02 .05 .03 /$ SR3 $54.02 .05 .03 /$ SR 36

$04.02 .05 .03 /$ SR39 $04.02 .05 .03 /$ SR40 $04.02 .05 .03 /$ SR 41

\#D 6.15 .1 .2 :

$04.02 .05 .01 /$ SR42 $04.02 .05 .01 /$ SR43 $04.02 .05 .01 /$ SR4 4

$04.02 .05 .01 /$ SR $4504.02 .05 .01 /$ SR4 $604.02 .05 .03 /$ SR2 6

$04.02 .05 .03 /$ SR3 $204.02 .05 .03 /$ SR3 $304.02 .05 .03 /$ SR3 4

$04.02 .05 .03 /$ SR3 $504.02 .05 .03 /$ SR3 $64.02 .05 .03 /$ SR3 7

$04.02 .05 .03 /$ SR39 $04.02 .05 .03 /$ SR40 $04.02 .05 .03 /$ SR4 1 


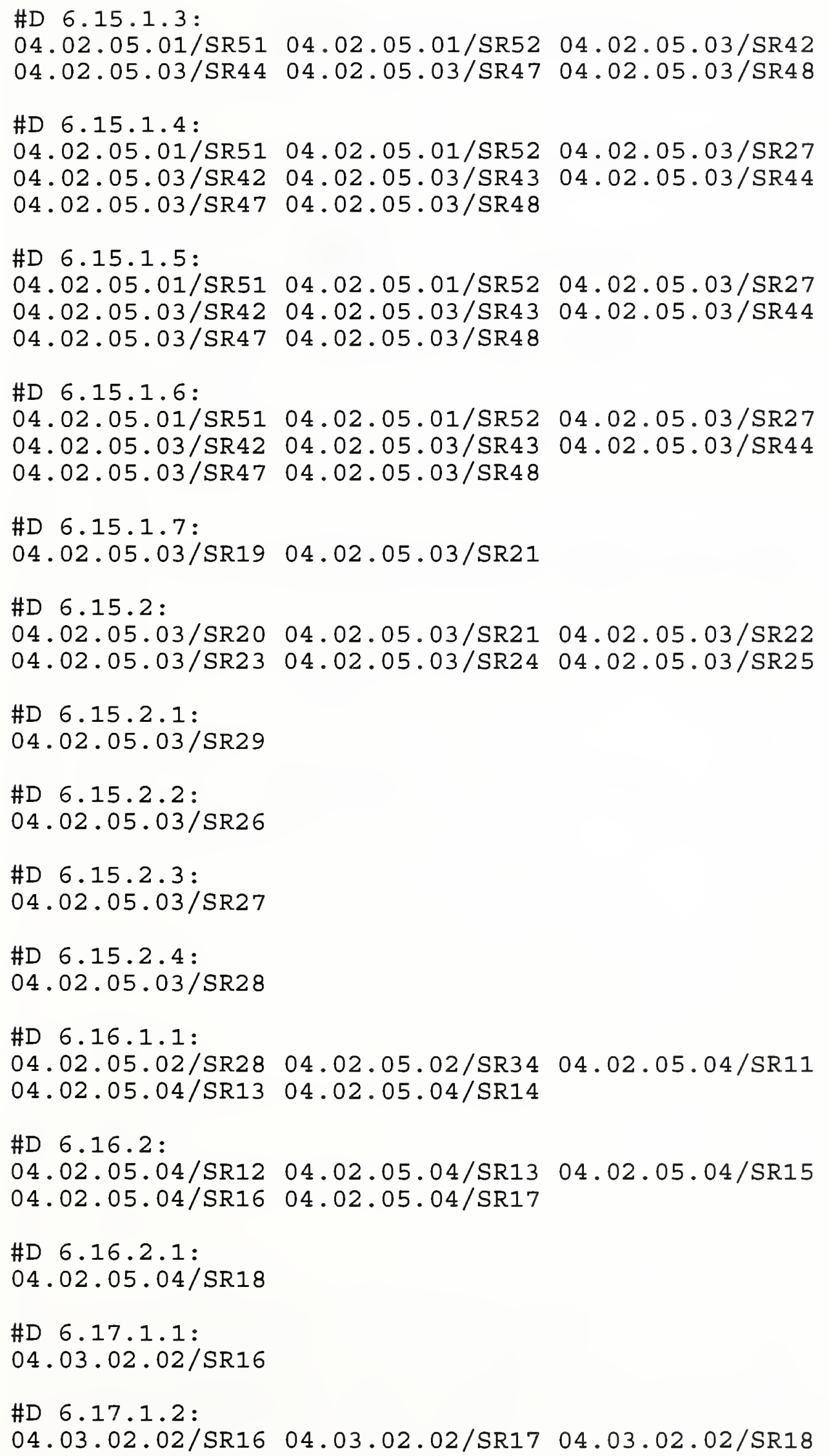




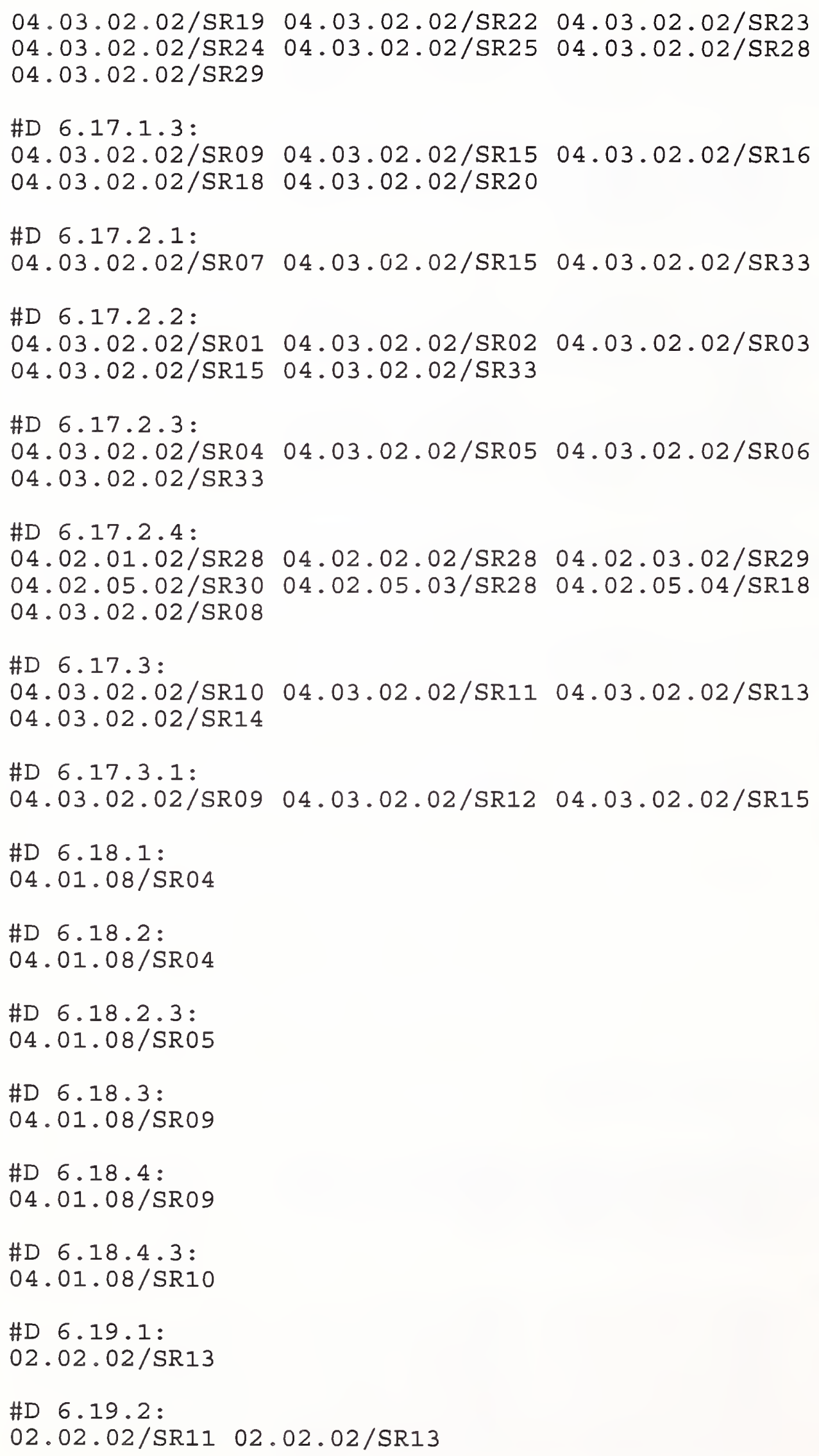


\#D $6.20:$

05.03/SR15 05.03/SR16 05.03/SR17 05.03/SR18

\#D 6.21.1:

$04.02 .01 .02 /$ SR17 $04.02 .01 .02 /$ SR18

\#D $6.21 .2:$

$04.02 .02 .02 /$ SR17 $04.02 .02 .02 /$ SR18

\#D $6.21 .3:$

$04.02 .03 .02 /$ SR17 $04.02 .03 .02 /$ SR18

\#D $6.21 .4:$

$04.02 .05 .02 /$ SR18 $04.02 .05 .02 /$ SR19

\#D 6.21.5:

$04.02 .05 .03 /$ SR17 $04.02 .05 .03 /$ SR18

\#D 6.21.6:

$04.02 .05 .04 /$ SR08 $04.02 .05 .04 /$ SR09 $04.02 .05 .04 /$ SR10

\#D 6.21.7:

$04.03 .02 .01 /$ SR0 $04.03 .02 .01 /$ SR0 8

\#D $6.21 .8:$

$06.02 .02 /$ SR12 $06.02 .02 /$ SR13

\#D $6.22:$

$05.02 /$ SR19 $05.02 /$ SR20 $05.02 /$ SR21 $05.02 / S R 2405.02 / S R 2505.02 / S R 26$

$05.02 /$ SR2 $705.02 /$ SR2 $805.02 / S R 2905.02 /$ SR $3105.02 /$ SR32

\#D 6.22.1:

$05.02 /$ SR0 7

\#D $6.22 .2:$

$05.02 /$ SR0 7

\#D $6.22 .3:$

$05.02 /$ SR0 7

\#D $6.22 .4:$

$05.02 /$ SR0 7

\#D $6.22 .5:$

$05.02 /$ SRO 7

\#D 7:

$02.01 .01 /$ SR08 $02.01 .02 .02 /$ SR01 $02.01 .02 .02 /$ SR02 $02.01 .02 .02 /$ SR03

$02.01 .02 .02 /$ SR0 $402.01 .02 .02 /$ SR0 $502.01 .02 .02 /$ SR0 6

$02.01 .02 .02 /$ SR0 $02.01 .02 .02 /$ SR08 $02.01 .02 .02 /$ SR09

$02.01 .03 .01 /$ SR01 $02.01 .03 .01 /$ SR02 $02.01 .03 .01 /$ SR03

$02.01 .03 .03 /$ SR01 $02.01 .03 .03 /$ SR02 $02.01 .03 .03 /$ SR03 $02.03 .03 /$ SR02

$02.03 .03 /$ SR0 $32.03 .03 /$ SR0 $402.03 .03 /$ SR0 $502.03 .03 /$ SRO 6 
\#D 7.1 :

$02.01 /$ SR02 $02.01 .01 /$ SR01 $02.01 .01 /$ SR02 $02.01 .01 /$ SR03

$02.01 .01 /$ SR04 02.01.01/SR05 02.01.01/SR06 $02.01 .01 /$ SR0 7

$02.01 .01 /$ SR09 02.01.01/SR10 02.01.02.01/SR01 02.01.02.01/SR03

$02.01 .02 .01 /$ SR0 $502.01 .02 .03 /$ SR01 $02.01 .02 .03 /$ SR02

$02.01 .03 .01 /$ SR01 $02.01 .03 .01 /$ SR02 $02.01 .03 .01 /$ SR0 3

$02.01 .03 .02 /$ SR01 $02.01 .03 .02 /$ SR02 $02.01 .03 .02 /$ SR03

$02.01 .03 .02 /$ SR0 $402.01 .03 .03 /$ SR01 $02.01 .03 .03 /$ SR0 2

$02.01 .03 .03 /$ SR03 $02.01 .03 .03 /$ SR0 $702.01 .03 .03 /$ SR0 $02.02 .01 /$ SR0 7

$02.02 .04 /$ SR08 $02.02 .04 /$ SR09 $02.02 .04 /$ SR10 $02.02 .04 /$ SR11

$02.02 .05 /$ SR01 $02.02 .05 /$ SR02 $02.02 .05 /$ SR0 $402.03 .01 /$ SR0 4

$02.03 .01 /$ SR0 $502.03 .01 /$ SR06 $02.03 .02 /$ SR01 $02.03 .02 /$ SR02

$02.03 .02 /$ SR0 $62.03 .03 /$ SR0 $02.03 .03 /$ SR0 $0203.03 /$ SR02 $03.03 /$ SR03

03.03 /SR0 $403.03 /$ SR0 $503.03 /$ SR0 $603.03 /$ SR0 $703.03 /$ SR0 $803.03 /$ SR 12

$03.03 /$ SR13 $03.03 /$ SR14 $03.03 /$ SR15 $03.03 /$ SR16 $03.03 /$ SR17 $03.03 /$ SR1 8

$03.03 /$ SR19 $03.04 /$ SR02 $03.04 /$ SR0 $303.04 /$ SR0 4

\#D 7.2 :

$02.01 .03 .01 /$ SR01 $02.01 .03 .01 /$ SR03 $02.01 .03 .02 /$ SR0 5

$02.01 .03 .02 /$ SR0 $02.01 .03 .03 /$ SR01 $02.01 .03 .03 /$ SR03 $05.03 /$ SR0 6

$05.03 /$ SR09 $05.03 /$ SR10 05.03/SR12

\#D 7.2.1:

05.03/SR01 05.03/SR04 05.03/SR07 05.03/SR11

\#D $7.3:$

$02.01 .02 .01 /$ SR0 $62.01 .02 .01 /$ SR0 $02.01 .02 .02 /$ SR11

$02.01 .02 .02 /$ SR12 $02.02 .01 /$ SR0 $702.02 .03 /$ SR02 $02.02 .03 /$ SR03

$02.02 .03 /$ SR0 $62.02 .04 /$ SR01 $02.02 .04 /$ SR02 $02.02 .04 /$ SR0 3

$02.02 .04 /$ SR0 $402.02 .04 /$ SR0 $502.02 .04 /$ SR0 $02.02 .04 /$ SR0 8

$02.02 .04 /$ SR11 $02.02 .05 /$ SR01 $02.02 .05 /$ SR02 $02.02 .05 /$ SR0 3

$02.02 .05 /$ SR0 $402.03 .01 /$ SR01 $02.03 .01 /$ SR02 $02.03 .01 /$ SR03

$02.03 .01 /$ SR0 $402.03 .01 /$ SR0 $502.03 .01 /$ SR0 $602.03 .02 /$ SR01

$02.03 .02 /$ SR0 $202.03 .02 /$ SR03 $02.03 .02 /$ SR0 $402.03 .02 /$ SR0 5

$02.03 .02 /$ SR0 $62.03 .02 /$ SR0 $02.03 .02 /$ SR08 $02.03 .03 /$ SR0 7

\#D 7.3.1:

$02.03 .03 /$ SR10

\#D 7.3.1.1:

$02.03 .03 /$ SR12 $04.01 .01 /$ SR01 $04.01 .01 /$ SR02 $04.01 .01 /$ SR03

$04.01 .01 /$ SR0 $704.02 .01 .01 /$ SR03 $04.02 .01 .02 /$ SR03 $04.03 .01 /$ SR0 7

$04.03 .04 .01 /$ SR15 $06.01 .02 /$ SR17 $06.01 .02 /$ SR 42

\#D 7.3.1.2:

$02.03 .03 /$ SR12 $04.01 .01 /$ SR04 $04.01 .01 /$ SR05 $04.01 .01 /$ SR0 6

$04.01 .01 /$ SR0 $704.02 .01 .01 /$ SR03 $04.02 .01 .02 /$ SR03 $04.03 .01 /$ SR0 7

$06.01 .02 /$ SR17 $06.01 .02 / S R 42$

\#D $7.3 \cdot 1 \cdot 3:$

$02.03 .03 /$ SR11 $04.01 .02 /$ SR01 $04.01 .02 /$ SR02 $04.01 .02 /$ SR03

$04.01 .02 /$ SR0 $704.02 .02 .01 /$ SR03 $04.02 .02 .02 /$ SR03 $04.03 .01 /$ SR0 7

$06.01 .02 /$ SR17 $06.01 .02 /$ SR 42 
\#D $7.3 .1 .4:$

$02.03 .03 /$ SR11 $04.01 .02 /$ SR0 $404.01 .02 /$ SR05 $04.01 .02 /$ SR0 6

$04.01 .02 /$ SR0 $04.02 .02 .01 /$ SR03 $04.02 .02 .02 /$ SR03 $04.03 .01 /$ SR0 7

$06.01 .02 /$ SR17 $06.01 .02 /$ SR 42

\#D $7.3 .1 .5:$

$02.03 .03 /$ SR15 $04.01 .03 /$ SR01 $04.01 .03 /$ SR02 $04.01 .03 /$ SR03

$04.01 .03 /$ SR09 $04.01 .03 /$ SR11 $04.02 .03 .01 /$ SR01 $04.02 .03 .01 /$ SR02

$04.02 .03 .01 /$ SR11 $04.02 .03 .02 /$ SR03 $04.03 .01 /$ SR0 $06.01 .02 /$ SR 17

$06.01 .02 / \mathrm{SR} 42$

\#D $7.3 .1 .6:$

$02.03 .03 /$ SR15 $04.01 .03 /$ SR04 04.01.03/SR05 $04.01 .03 /$ SR0 6

$04.01 .03 /$ SR09 $04.01 .03 /$ SR10 $04.02 .03 .01 /$ SR01 $04.02 .03 .01 /$ SR02

$04.02 .03 .01 /$ SR11 $04.02 .03 .02 /$ SR03 $04.03 .01 /$ SR0 $06.01 .02 /$ SR17

$06.01 .02 /$ SR 42

\#D 7.3.1.7:

$02.03 .03 /$ SR16 04.01.04/SR01 $04.01 .04 /$ SR02 $04.01 .04 /$ SR03

$04.01 .04 /$ SR0 $04.01 .04 /$ SR09 $04.02 .04 .01 /$ SR01 04.02.04.01/SR02

$04.02 .04 .01 /$ SR0 $54.02 .04 .02 /$ SR01 $04.03 .01 /$ SR0 $06.01 .02 /$ SR 17

$06.01 .02 /$ SR 42

\#D $7.3 .1 .8:$

$02.03 .03 /$ SR16 $04.01 .04 /$ SR04 $04.01 .04 /$ SR05 $04.01 .04 /$ SR0 6

$04.01 .04 /$ SR07 04.01.04/SR08 04.01.04/SR09 04.02.04.01/SR01

$04.02 .04 .01 /$ SR02 $04.02 .04 .01 /$ SR05 $04.02 .04 .02 /$ SR01 $04.03 .01 /$ SR0 7

$06.01 .02 /$ SR17 $06.01 .02 /$ SR 42

\#D 7.3.1.9:

$02.03 .03 /$ SR13 $04.01 .05 /$ SR01 $04.01 .05 /$ SR02 $04.01 .05 /$ SR03

$04.01 .05 /$ SR0 $04.01 .05 /$ SR08 $04.02 .05 .01 /$ SR03 $04.02 .05 .02 /$ SR03

$04.03 .01 /$ SR0 $706.01 .02 /$ SR17 $06.01 .02 /$ SR 42

\#D $7.3 .1 .10:$

$02.03 .03 /$ SR13 $04.01 .05 /$ SR04 04.01.05/SR05 04.01.05/SR0 6

$04.01 .05 /$ SR0 $704.01 .05 /$ SR0 $804.02 .05 .01 /$ SR03 $04.02 .05 .02 /$ SR03

$04.03 .01 /$ SR07 $06.01 .02 /$ SR17 $06.01 .02 /$ SR 42

\#D $7.3 .1 .11:$

$02.03 .03 /$ SR14 $04.01 .06 /$ SR01 $04.01 .06 /$ SR02 $04.01 .06 /$ SR03

$04.01 .06 /$ SR07 $04.01 .06 /$ SR08 $04.02 .05 .01 /$ SR0 $404.02 .05 .02 /$ SR0 4

$04.02 .05 .03 /$ SR03 $04.03 .01 /$ SR0 $06.01 .02 /$ SR17 $06.01 .02 / S R 42$

\#D $7.3 .1 .12:$

$02.03 .03 /$ SR14 $04.01 .06 /$ SR04 $04.01 .06 /$ SR05 $04.01 .06 /$ SR0 6

$04.01 .06 /$ SR0 $704.01 .06 /$ SR08 $04.02 .05 .01 /$ SR0 $404.02 .05 .02 /$ SR0 4

$04.02 .05 .03 /$ SR03 $04.03 .01 /$ SR0 $706.01 .02 /$ SR17 $06.01 .02 /$ SR 42

\#D $7.3 .1 .13:$

$02.03 .03 /$ SR17 $04.01 .07 /$ SR01 $04.01 .07 /$ SR02 $04.01 .07 /$ SR03

$04.01 .07 /$ SR0 $404.01 .07 /$ SR05 $04.01 .07 /$ SR11 $04.01 .07 /$ SR12

$04.01 .07 /$ SR13 04.01.07/SR14 06.01.02/SR17 $06.01 .02 /$ SR4 2 


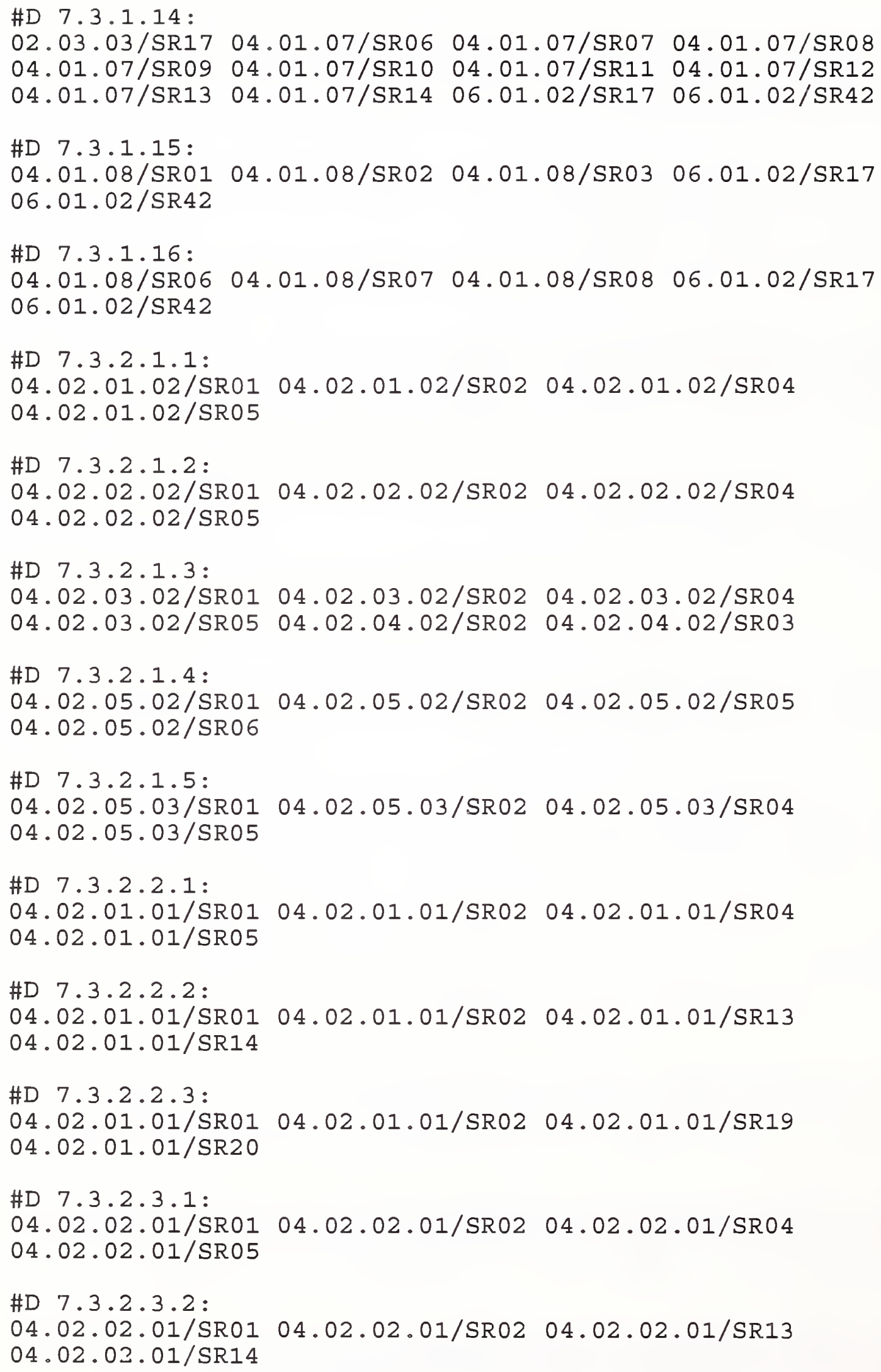


\#D $7 \cdot 3 \cdot 2 \cdot 3 \cdot 3:$

$04.02 .02 .01 /$ SR01 $04.02 .02 .01 /$ SR02 $04.02 .02 .01 /$ SR20

$04.02 .02 .01 /$ SR2 1

\#D 7.3.2.4.1:

$04.02 .03 .01 /$ SR09 $04.02 .03 .01 /$ SR10 $04.02 .03 .01 /$ SR12

$04.02 .03 .01 /$ SR13 $04.02 .04 .01 /$ SR0 6

\#D 7.3.2.4.2:

$04.02 .03 .01 /$ SR09 $04.02 .03 .01 /$ SR10 $04.02 .03 .01 /$ SR2 5

$04.02 .03 .01 /$ SR2 $64.02 .03 .01 /$ SR2 $704.02 .04 .01 /$ SR 18

$04.02 .04 .01 /$ SR 19

\#D $7.3 .2 .4 .3:$

$04.02 .03 .01 /$ SR09 $04.02 .03 .01 /$ SR10 $04.02 .03 .01 /$ SR 34

$04.02 .03 .01 /$ SR $3504.02 .04 .01 /$ SR2 6

\#D $7.3 .2 .4 .4:$

$04.02 .03 .01 /$ SR09 $04.02 .03 .01 /$ SR10 $04.02 .03 .01 /$ SR 40

$04.02 .03 .01 /$ SR $4104.02 .04 .01 /$ SR 32

\#D $7.3 .2 .4 .5:$

$04.02 .03 .01 /$ SR09 $04.02 .03 .01 /$ SR10 $04.02 .03 .01 /$ SR 46

$04.02 .03 .01 /$ SR $4704.02 .04 .01 /$ SR 37

\#D $7.3 .2 .4 .6:$

$02.03 .03 /$ SR15 $04.02 .03 .01 /$ SR09 $04.02 .03 .01 /$ SR10 $04.02 .03 .01 /$ SR52 $04.02 .03 .01 /$ SR5 8

\#D $7.3 \cdot 2 \cdot 4 \cdot 7:$

$02.03 .03 /$ SR15 $04.02 .03 .01 /$ SR09 $04.02 .03 .01 /$ SR10 $04.02 .03 .01 /$ SR79 $04.02 .03 .01 /$ SR 80

\#D $7.3 .2 .4 .8:$

$02.03 .03 /$ SR15 $04.02 .03 .01 /$ SR09 $04.02 .03 .01 /$ SR10 $04.02 .03 .01 /$ SR 64 $04.02 .03 .01 /$ SR $6504.02 .03 .01 /$ SR 66

\#D $7.3 .2 .4 .9:$

$02.03 .03 /$ SR15 $04.02 .03 .01 /$ SR09 $04.02 .03 .01 /$ SR10 $04.02 .03 .01 /$ SR72 $04.02 .03 .01 /$ SR73 $04.02 .03 .01 /$ SR74

\#D 7.3 .2 .5 .1 :

$04.02 .04 .01 /$ SR0 $304.02 .04 .01 /$ SR0 $404.02 .04 .01 / S R 42$

$04.02 .04 .01 /$ SR 48

\#D 7.3.2.5.2:

$04.02 .04 .01 /$ SR03 $04.02 .04 .01 /$ SR04 $04.02 .04 .01 /$ SR 70

$04.02 .04 .01 /$ SR 71

\#D $7.3 \cdot 2 \cdot 5 \cdot 3:$

$04.02 .04 .01 /$ SR0 $304.02 .04 .01 /$ SR0 $404.02 .04 .01 /$ SR55

$04.02 .04 .01 /$ SR56 $04.02 .04 .01 /$ SR 57

\#D 7.3.2.5.4: 


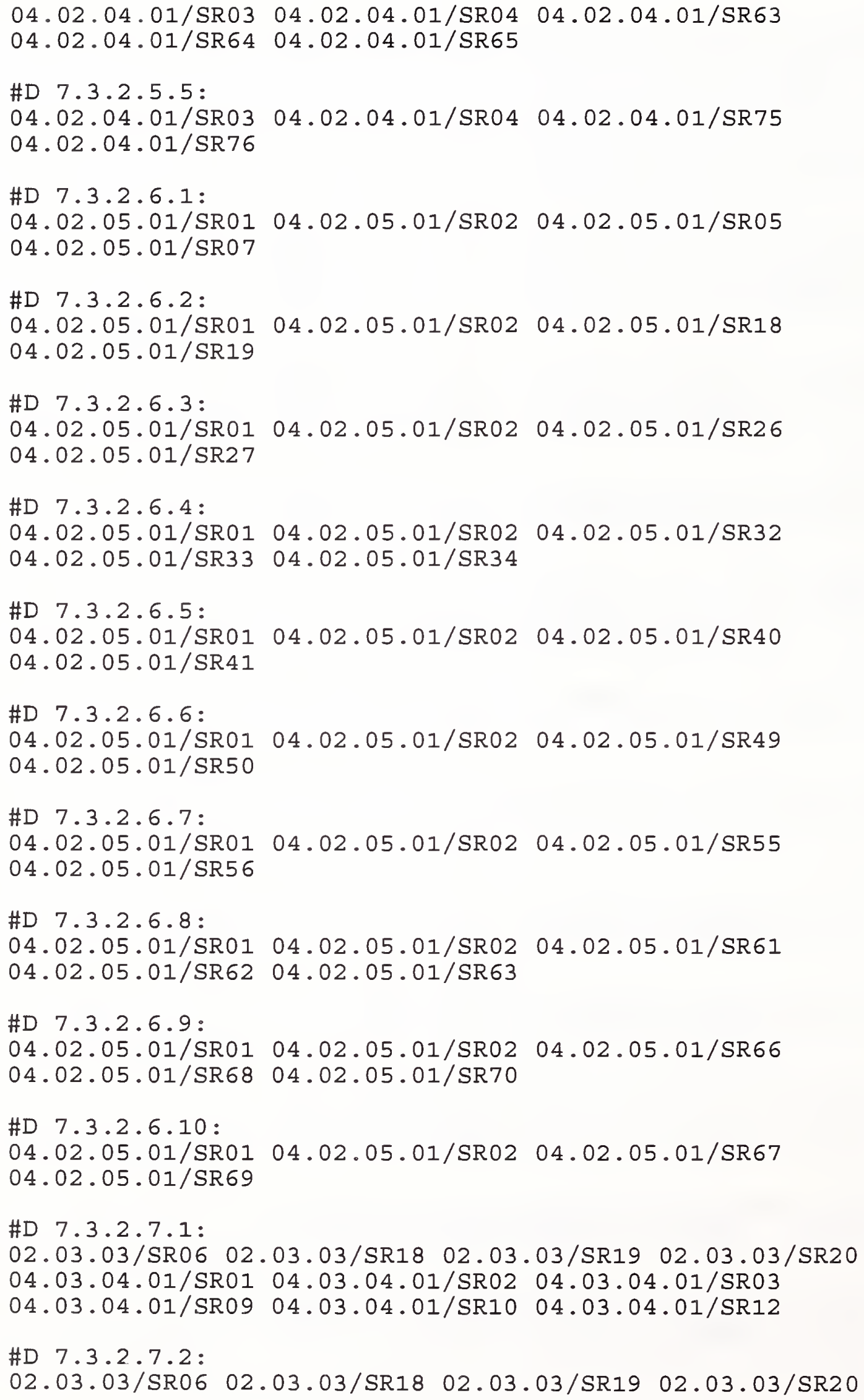


$04.03 .04 .01 /$ SR0 $404.03 .04 .01 /$ SR0 $504.03 .04 .01 /$ SR0 6

$04.03 .04 .01 /$ SR09 $04.03 .04 .01 /$ SR10 $04.03 .04 .01 /$ SR13

\#D 7.3.2.8:

$04.03 .01 /$ SR01 $04.03 .01 /$ SR02 $04.03 .01 /$ SR03 $04.03 .01 /$ SR0 4

$04.03 .01 /$ SR05 $04.03 .01 /$ SR06

\#D $7.3 .2 .9:$

$04.03 .03 /$ SR01 $04.03 .03 /$ SR02 $04.03 .03 /$ SR03

\#D $7.3 .2 .10:$

$06.02 .02 /$ SR01 $06.02 .02 /$ SR02 $06.02 .02 /$ SR03

\#D $7.3 .3 .1:$

$02.03 .03 /$ SR10 06.01.01/SR01 $06.01 .01 /$ SR02 $06.01 .02 /$ SR01 $06.01 .02 /$ SR02 $06.01 .02 /$ SR03 $06.01 .02 /$ SR0 $706.01 .02 /$ SR0 8 $06.01 .02 /$ SR18

\#D $7.3 .3 .2:$

$02.03 .03 /$ SR10 06.01.01/SR01 06.01.01/SR02 $06.01 .02 /$ SR04 $06.01 .02 /$ SR05 $06.01 .02 /$ SR0 $606.01 .02 /$ SR0 $06.01 .02 /$ SR18

\#D $7.3 .3 .3:$

$02.03 .03 /$ SR10 06.01.01/SR01 06.01.01/SR02 $06.01 .02 /$ SR09 06.01.02/SR10 06.01.02/SR11 06.01.02/SR15 $06.01 .02 /$ SR18

\#D $7.3 .3 .4:$

$02.03 .03 /$ SR10 06.01.01/SR01 $06.01 .01 /$ SR02 $06.01 .02 /$ SR12 $06.01 .02 /$ SR13 $06.01 .02 /$ SR14 $06.01 .02 /$ SR18

\#D $7.3 \cdot 3 \cdot 5:$

$02.03 .03 /$ SR10 $06.01 .02 /$ SR19 $06.01 .02 /$ SR24 $06.01 .02 /$ SR2 5

$06.01 .02 /$ SR2 $606.01 .02 /$ SR40 $06.01 .02 / S R 4106.01 .02 / S R 45$

$06.01 .02 /$ SR46 $06.01 .02 /$ SR $4706.01 .02 /$ SR 48

\#D 7.3.3.6:

$02.03 .03 /$ SR10 $06.01 .02 /$ SR19 $06.01 .02 /$ SR2 $706.01 .02 /$ SR2 8

$06.01 .02 /$ SR29 $06.01 .02 /$ SR40 $06.01 .02 /$ SR4 1

\#D $7.3 .3 .7:$

$06.01 .02 /$ SR30 $06.01 .02 /$ SR31 $06.01 .02 /$ SR32 $06.01 .02 /$ SR3 6

\#D $7.3 .3 .8:$

$02.03 .03 /$ SR10 $06.01 .02 /$ SR33 $06.01 .02 /$ SR34 $06.01 .02 /$ SR3 5 $06.01 .02 /$ SR 49

\#D $7.3 .4:$

$02.01 /$ SR01 $02.01 /$ SR03 $02.01 /$ SR04 $02.01 /$ SR0 $502.01 /$ SR0 $602.01 / S R 07$

$02.01 /$ SR0 8 02.01/SR09 $02.01 /$ SR10 $02.01 /$ SR11 $02.01 /$ SR12 $02.01 / S R 13$

$02.01 /$ SR14 02.01.02.01/SR02 02.01.02.01/SR06 02.01.02.01/SR07

$02.01 .02 .02 /$ SR01 $02.01 .02 .02 /$ SR02 $02.01 .02 .02 /$ SR0 3

$02.01 .02 .02 /$ SR0 $402.01 .02 .02 /$ SR0 $502.01 .02 .02 /$ SR0 6

$02.01 .02 .02 /$ SR0 $702.01 .02 .02 /$ SR0 $02.01 .02 .02 /$ SR11

$02.01 .02 .02 /$ SR12 $02.01 .03 .01 /$ SR01 $02.01 .03 .02 /$ SR01 


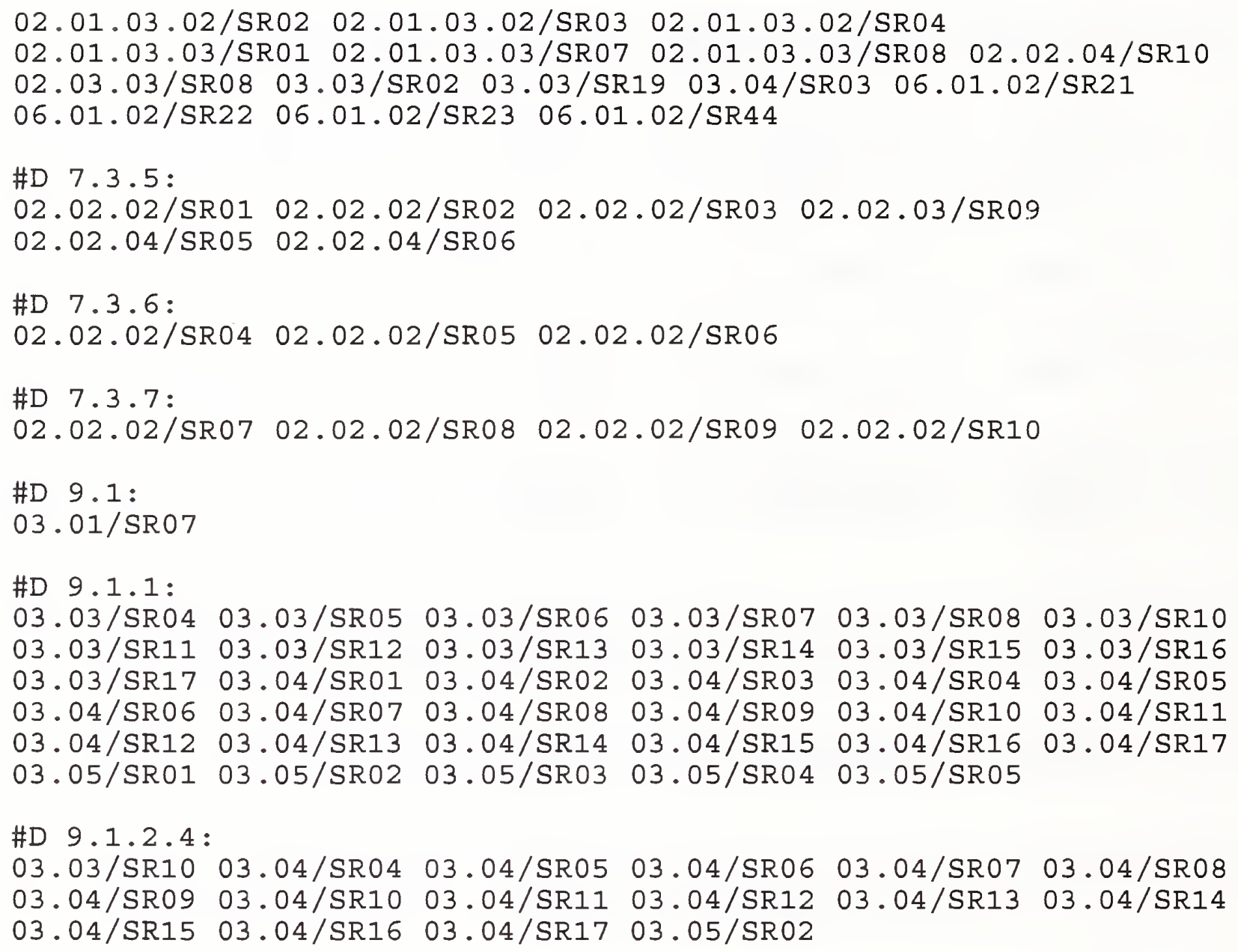




\section{APPENDIX I}

\section{STANDARD SPECIFICATIONS CROSS-REFERENCE}

\#S $4.1 .1 / 15 / 1:$

$04.02 .01 .01 /$ SR10 $04.02 .01 .02 /$ SR3 $804.02 .02 .01 /$ SR10

$04.02 .02 .02 / S R 3804.02 .03 .01 / S R 2104.02 .03 .02 / S R 40$

$04.02 .04 .01 /$ SR14 $04.02 .04 .01 /$ SR $8204.02 .04 .02 /$ SR13

$04.02 .05 .01 /$ SR11 $04.02 .05 .01 /$ SR23 $04.02 .05 .01 /$ SR 46

$04.02 .05 .02 /$ SR49 $04.02 .05 .03 /$ SR4 $104.03 .02 .02 /$ SR2 9

\#S $4.1 .2 / 15 / 2:$

$04.02 .01 .01 /$ SR09 $04.02 .01 .02 /$ SR37 $04.02 .02 .01 /$ SR0 9

$04.02 .02 .02 /$ SR3 $704.02 .03 .01 /$ SR2 $104.02 .03 .02 / S R 40$

$04.02 .04 .01 /$ SR14 $04.02 .04 .01 /$ SR81 $04.02 .04 .02 /$ SR13

$04.02 .05 .01 /$ SR22 $04.02 .05 .01 / S R 4504.02 .05 .03 / S R 40$

\#S $4.4 .1 / 22 / 1:$

$02.01 .01 /$ SR09

\#S $4.4 .1 / 22 / 3:$

$04.01 .01 /$ SR01 $04.01 .01 /$ SR04 $04.01 .02 /$ SR01 $04.01 .02 /$ SR0 4

$04.01 .03 /$ SR01 $04.01 .03 /$ SR0 $404.01 .04 /$ SR01 $04.01 .04 /$ SR0 4

$04.01 .05 /$ SR01 $04.01 .05 /$ SR04 $04.01 .06 /$ SR01 $04.01 .06 /$ SR0 4

04.01.07/SR01 04.01.07/SR06 04.01.08/SR01 04.01.08/SR06

\#S $4.4 .1 / 23 / 1$ :

$04.02 .01 .01 /$ SR0 $404.02 .01 .01 /$ SR13 $04.02 .01 .01 /$ SR19

$04.02 .01 .02 /$ SR0 $404.02 .01 .02 /$ SR0 $504.02 .02 .01 /$ SR0 4

$04.02 .02 .01 /$ SR13 $04.02 .02 .01 /$ SR20 $04.02 .02 .02 /$ SR0 4

$04.02 .02 .02 /$ SR05 $04.02 .03 .01 /$ SR12 $04.02 .03 .01 /$ SR2 5

$04.02 .03 .01 /$ SR34 $04.02 .03 .01 /$ SR $4004.02 .03 .01 /$ SR 46

$04.02 .03 .01 /$ SR52 $04.02 .03 .01 /$ SR $6404.02 .03 .01 /$ SR72

$04.02 .03 .01 /$ SR79 $04.02 .03 .02 /$ SR0 $404.02 .03 .02 /$ SR0 5

$04.02 .04 .01 /$ SR42 $04.02 .04 .01 /$ SR55 $04.02 .04 .01 /$ SR 63

$04.02 .04 .01 /$ SR70 $04.02 .04 .01 /$ SR75 $04.02 .04 .02 /$ SR02

$04.02 .04 .02 /$ SR03 $04.02 .05 .01 /$ SR0 $504.02 .05 .01 /$ SR18

$04.02 .05 .01 /$ SR2 $604.02 .05 .01 /$ SR32 $04.02 .05 .01 /$ SR4 0

$04.02 .05 .01 /$ SR49 $04.02 .05 .01 /$ SR55 $04.02 .05 .01 /$ SR61

$04.02 .05 .01 /$ SR6 $64.02 .05 .01 /$ SR $6704.02 .05 .02 /$ SR0 5

$04.02 .05 .02 /$ SR0 $64.02 .05 .03 /$ SR0 $404.02 .05 .03 /$ SR0 $504.03 .03 /$ SR01

$04.03 .04 .01 /$ SR01 $04.03 .04 .01 /$ SR0 4 
\#S $4.4 .2 / 24 / 3:$

$02.01 /$ SRO1

\#S $4.4 .2 / 25 / 2$ :

$02.01 .01 /$ SR01 02.01.01/SR02 $02.01 .01 /$ SR03 $02.01 .01 /$ SR0 4

$02.01 .01 /$ SR05 02.01.01/SR06 02.01.01/SR07 $02.01 .01 /$ SR0 8

$02.01 .01 /$ SR10 $03.03 /$ SR19

\#S $4.4 .2 / 25 / 3:$

$02.01 .02 .01 / \mathrm{SR} 03$

\#S $4.4 .3 / 25 / 4:$

$05.02 /$ SR10 $05.03 /$ SR01 $05.03 /$ SR02

\#S $4.4 .3 / 25 / 5:$

$02.01 .01 /$ SR04 05.03/SR04 05.03/SR05 05.03/SR06 05.03/SR07

$05.03 /$ SR0 8

\#S $4.4 .3 / 25 / 8:$

05.03 /SR03 $05.03 /$ SR14

\#S $4.4 .3 / 26 / 2:$

$04.02 .01 .01 /$ SR11 $04.02 .01 .01 /$ SR17 $04.02 .01 .01 /$ SR2 3

$04.02 .01 .02 /$ SR0 8 $04.02 .02 .01 /$ SR11 $04.02 .02 .01 /$ SR18

$04.02 .02 .01 /$ SR2 $404.02 .02 .02 /$ SR0 $804.02 .03 .01 /$ SR2 3

$04.02 .03 .01 /$ SR32 $04.02 .03 .01 /$ SR3 $804.02 .03 .01 /$ SR 44

$04.02 .03 .01 /$ SR50 $04.02 .03 .01 /$ SR62 $04.02 .03 .01 /$ SR70

$04.02 .03 .01 /$ SR7 $704.02 .03 .01 /$ SR82 $04.02 .03 .02 /$ SR0 8

$04.02 .04 .01 /$ SR16 $04.02 .04 .01 /$ SR2 $404.02 .04 .01 /$ SR3 0

$04.02 .04 .01 /$ SR3 $504.02 .04 .01 /$ SR40 $04.02 .04 .01 /$ SR5 3

$04.02 .04 .01 /$ SR61 $04.02 .04 .01 /$ SR68 $04.02 .04 .01 /$ SR73

$04.02 .04 .01 /$ SR83 $04.02 .04 .02 /$ SR06 $04.02 .05 .01 /$ SR 16

$04.02 .05 .01 /$ SR2 $404.02 .05 .01 /$ SR3 $004.02 .05 .01 /$ SR 38

$04.02 .05 .01 /$ SR $4704.02 .05 .01 /$ SR53 $04.02 .05 .01 /$ SR59

$04.02 .05 .01 /$ SR64 $04.02 .05 .01 /$ SR80 $04.02 .05 .01 /$ SR8 2

$04.02 .05 .02 /$ SR09 $04.02 .05 .03 /$ SR0 $804.03 .01 /$ SR 10

\#S 4.4.3/28/1:

$04.02 .01 .01 /$ SR11

$04.02 .01 .01 /$ SR18

$04.02 .01 .02 /$ SR0 8

$04.02 .02 .01 /$ SR12

$04.02 .02 .01 /$ SR2 4

$04.02 .02 .02 /$ SR09

$04.02 .03 .01 /$ SR 32

$04.02 .03 .01 /$ SR39

$04.02 .03 .01 /$ SR50

$04.02 .03 .01 /$ SR 63

$04.02 .03 .01 /$ SR77

$04.02 .03 .01 /$ SR8 3

$04.02 .04 .01 /$ SR16

$04.02 .04 .01 /$ SR2 5

$04.02 .04 .01 /$ SR 35
$04.02 .01 .01 /$ SR12

$04.02 .01 .01 /$ SR2 3

$04.02 .01 .02 /$ SR09

$04.02 .02 .01 /$ SR18

$04.02 .02 .01 /$ SR2 5

$04.02 .03 .01 /$ SR2 3

$04.02 .03 .01 /$ SR3 3

$04.02 .03 .01 / S R 44$

$04.02 .03 .01 /$ SR5 1

$04.02 .03 .01 /$ SR70

$04.02 .03 .01 /$ SR78

$04.02 .03 .02 /$ SR0 8

$04.02 .04 .01 /$ SR1 7

$04.02 .04 .01 /$ SR3 0

$04.02 .04 .01 /$ SR 36
$04.02 .01 .01 /$ SR1 7

$04.02 .01 .01 /$ SR2 4

$04.02 .02 .01 /$ SR11

$04.02 .02 .01 /$ SR19

$04.02 .02 .02 /$ SR0 8

$04.02 .03 .01 / \mathrm{SR} 24$

$04.02 .03 .01 /$ SR3 8

$04.02 .03 .01 /$ SR 45

$04.02 .03 .01 /$ SR62

$04.02 .03 .01 /$ SR71

$04.02 .03 .01 /$ SR 82

$04.02 .03 .02 /$ SR09

$04.02 .04 .01 / \mathrm{SR} 24$

$04.02 .04 .01 /$ SR3 1

$04.02 .04 .01 /$ SR 40 


\begin{abstract}
$04.02 .04 .01 /$ SR41 $04.02 .04 .01 /$ SR53 $04.02 .04 .01 /$ SR5 4
$04.02 .04 .01 /$ SR61 $04.02 .04 .01 /$ SR62 $04.02 .04 .01 /$ SR 68

$04.02 .04 .01 /$ SR69 $04.02 .04 .01 /$ SR73 $04.02 .04 .01 /$ SR74

$04.02 .04 .01 /$ SR83 $04.02 .04 .01 /$ SR8 $404.02 .04 .02 /$ SR0 6

$04.02 .04 .02 /$ SR0 $704.02 .05 .01 /$ SR16 $04.02 .05 .01 /$ SR 17

$04.02 .05 .01 /$ SR2 $404.02 .05 .01 /$ SR2 $504.02 .05 .01 /$ SR30

$04.02 .05 .01 /$ SR31 $04.02 .05 .01 /$ SR3 $04.02 .05 .01 /$ SR39

$04.02 .05 .01 /$ SR4 $704.02 .05 .01 /$ SR48 $04.02 .05 .01 /$ SR5 3

$04.02 .05 .01 /$ SR54 $04.02 .05 .01 /$ SR59 $04.02 .05 .01 /$ SR60

$04.02 .05 .01 /$ SR64 $04.02 .05 .01 /$ SR65 $04.02 .05 .01 /$ SR 80

$04.02 .05 .01 /$ SR81 $04.02 .05 .01 /$ SR82 $04.02 .05 .01 /$ SR83

$04.02 .05 .02 /$ SR09 $04.02 .05 .02 /$ SR10 $04.02 .05 .03 /$ SR0 8

$04.02 .05 .03 /$ SR09 $04.03 .01 /$ SR10 $04.03 .01 /$ SR11 $04.03 .04 .01 /$ SR 14

$06.01 .02 /$ SR21 $06.01 .02 / S R 44$
\end{abstract}

\#S $4.4 .4 / 28 / 4$ :

$02.03 .02 / \mathrm{SR} 04$

\#S $4.4 .4 / 28 / 5$ :

$02.01 .01 /$ SR01 $02.02 .01 /$ SR01 $02.02 .01 /$ SR02 $02.02 .01 /$ SR0 4

$02.02 .01 /$ SR05 $02.02 .01 /$ SR06

\#S $4.4 .4 / 28 / 6$ :

$02.02 .01 /$ SR03 $02.02 .01 /$ SR08

\#S $4.4 .4 / 28 / 7$ :

$02.02 .02 /$ SR01 $02.02 .03 /$ SR07 $02.02 .03 /$ SR08 $02.02 .03 /$ SR09

$02.02 .03 /$ SR 10

\#S $4.4 .4 / 29 / 1$ :

$02.02 .03 /$ SR01 $02.02 .03 /$ SR02 $02.02 .03 /$ SR03 $02.02 .03 /$ SR0 4

\#S $4.4 .4 / 29 / 2:$

$02.02 .05 /$ SR01 $02.02 .05 /$ SR02 $02.02 .05 /$ SR03 $02.02 .05 /$ SR0 4

\#S $4.4 .4 / 29 / 3:$

$02.02 .04 /$ SR01 $02.02 .04 /$ SR02 $02.02 .04 /$ SR03 $02.02 .04 /$ SR0 4

$02.02 .04 /$ SR05 $02.02 .04 /$ SR06 $02.02 .04 /$ SR0 7

\#S $4.4 .4 / 29 / 4:$

$02.01 .01 /$ SR02 $02.02 .04 /$ SR08 $02.02 .04 /$ SR09 $02.02 .04 /$ SR 10 $02.02 .04 /$ SR 11

\#S $4.4 .4 / 29 / 5:$

$02.02 .02 / \mathrm{SR} 02$

$02.02 .02 / \mathrm{SRO} 3$

$02.02 .02 /$ SR0 $502.02 .02 /$ SR0 6

$02.02 .02 /$ SRO 9

$02.02 .02 /$ SR 10

$02.03 .01 /$ SRO 1

$02.03 .01 /$ SRO2

$02.03 .01 / \mathrm{SRO} 3$

$04.01 .01 /$ SRO2

$04.01 .01 /$ SR0 3

$04.01 .01 /$ SR0 5

$04.01 .01 /$ SR0 6

$04.01 .02 /$ SRO2

$04.01 .02 /$ SR03

$04.01 .02 /$ SR0 5

$04.01 .02 /$ SR0 6

$04.01 .03 /$ SR02

$04.01 .03 /$ SR03

$04.01 .03 /$ SR0 5

$04.01 .03 /$ SR06

$04.01 .04 /$ SRO2

$04.01 .04 /$ SR03

$04.01 .04 /$ SR0 5

$04.01 .04 /$ SR0 6

$04.01 .05 /$ SR0 2

$04.01 .05 /$ SR03

$04.01 .05 /$ SR0 5

$04.01 .05 /$ SR0 6

$04.01 .06 /$ SR0 2

$04.01 .06 /$ SR0 3

$04.01 .06 /$ SR0 5

$04.01 .06 /$ SR0 6

$04.01 .07 /$ SR02

$04.01 .07 /$ SR0 3

$04.01 .07 /$ SR0 7

$04.01 .07 /$ SR0 8

$04.01 .08 /$ SRO 2

$04.01 .08 /$ SR0 3

$04.01 .08 /$ SR0 7 
04.01.08/SR08 04.02.01.01/SR01 04.02.01.01/SR02 04.02.01.02/SR01 $04.02 .01 .02 /$ SR02 $04.02 .02 .01 /$ SR01 $04.02 .02 .01 /$ SR02

$04.02 .02 .02 /$ SR01 $04.02 .02 .02 /$ SR02 $04.02 .03 .01 /$ SR0 9

$04.02 .03 .01 /$ SR10 $04.02 .03 .02 /$ SR01 $04.02 .03 .02 /$ SR0 2

$04.02 .04 .01 /$ SR0 3 $04.02 .04 .01 /$ SR0 $404.02 .05 .01 /$ SR01

$04.02 .05 .01 /$ SR02 $04.02 .05 .02 /$ SR01 $04.02 .05 .02 /$ SR02

$04.02 .05 .03 /$ SR01 $04.02 .05 .03 /$ SR02 $04.03 .01 /$ SR02 $04.03 .01 /$ SR03

$04.03 .03 /$ SR02 04.03.03/SR03 04.03.04.01/SR02 04.03.04.01/SR03

$04.03 .04 .01 /$ SR05 $04.03 .04 .01 /$ SR0 $606.01 .02 /$ SR02 $06.01 .02 /$ SR03

$06.01 .02 /$ SR05 $06.01 .02 /$ SR06 $06.01 .02 /$ SR10 $06.01 .02 /$ SR11

$06.01 .02 /$ SR13 06.01.02/SR14 $06.01 .02 /$ SR25 $06.01 .02 /$ SR2 6

$06.01 .02 /$ SR28 $06.01 .02 /$ SR29 $06.01 .02 /$ SR31 $06.01 .02 /$ SR32

$06.01 .02 /$ SR3 $406.01 .02 /$ SR35 $06.02 .02 /$ SR02 $06.02 .02 /$ SR0 3

\#S $4.4 .5 / 29 / 7$ :

$02.01 .02 .01 /$ SR01 $02.01 .02 .01 /$ SR02 $02.01 .02 .01 /$ SR0 6

$02.01 .02 .01 /$ SR07 $02.01 .02 .02 /$ SRI1 $02.01 .02 .02 /$ SR12

$02.01 .02 .03 /$ SR01 $02.01 .02 .03 /$ SR02

\#S $4.4 .5 / 29 / 8$ :

$02.01 .02 .02 /$ SR01 $02.01 .02 .02 /$ SR02 $02.01 .02 .02 /$ SR03

$02.01 .02 .02 /$ SR0 $402.01 .02 .02 /$ SR0 $502.01 .02 .02 /$ SR0 6

$02.01 .02 .02 /$ SR07 $02.01 .02 .02 /$ SR0 $802.01 .02 .02 /$ SR0 9

$02.01 .02 .02 /$ SR11 $02.01 .02 .02 /$ SR12

\#S $4.4 .5 / 29 / 9$ :

$02.01 .02 .01 /$ SR0 $402.01 .02 .02 /$ SR10

\#S $4.4 .5 / 30 / 1$ :

$02.01 .01 /$ SR05 $02.01 .01 /$ SR07 $02.01 .03 .01 /$ SR01 $02.01 .03 .01 / S R 02$

$02.01 .03 .01 /$ SR03 $02.01 .03 .02 /$ SR01 $02.01 .03 .02 /$ SR02

$02.01 .03 .02 /$ SR03 $02.01 .03 .02 /$ SR0 $402.01 .03 .02 /$ SR0 5

$02.01 .03 .02 /$ SR0 $62.01 .03 .03 /$ SR01 $02.01 .03 .03 /$ SR02

$02.01 .03 .03 /$ SR03 $02.01 .03 .03 /$ SR0 $02.01 .03 .03 /$ SR0 8

\#S $4.4 .5 / 30 / 2:$

$02.01 /$ SR02 $02.01 .01 /$ SR09

\#S $4.4 .6 / 30 / 4:$

$02.03 .01 /$ SR0 $402.03 .01 /$ SR05 $02.03 .01 /$ SR0 6

\#S $4.4 .6 / 30 / 5:$

02.01/SR03 02.01/SR04 02.01/SR05 02.01/SR06 02.01/SR09 02.01/SR10

$02.01 /$ SR11 $02.01 /$ SR12 $02.01 /$ SR13 $02.01 /$ SR14

\#S $4.4 .6 / 30 / 6:$

$02.03 .02 /$ SR01 $02.03 .02 /$ SR02 $02.03 .02 /$ SR03 $02.03 .02 /$ SR0 4

02.03 .02 /SR05 $02.03 .02 /$ SR0 $602.03 .02 /$ SR0 $02.03 .02 /$ SR0 8

\#S $4.4 .6 / 30 / 9:$

$02.03 .03 /$ SR02 $02.03 .03 /$ SR03 $02.03 .03 /$ SR0 $402.03 .03 /$ SR0 6

$02.03 .03 /$ SR0 7

\#S $4.4 .6 / 31 / 1$ : 
$02.03 .03 /$ SR0 $602.03 .03 /$ SR09

\#S 4.4.6/31/2:

$02.03 .03 /$ SR 15

\#S $4.4 .6 / 31 / 3$ :

$02.03 .03 /$ SR16

\#S $4.4 .6 / 31 / 4$ :

$02.03 .03 /$ SR0 4

\#S $4.4 .6 / 31 / 5$ :

$02.03 .03 /$ SR04 $02.03 .03 /$ SR0 8

\#S 4.4.6/31/6:

$02.03 .03 /$ SR0 5

\#S 4.4.6/31/9:

$02.03 .03 /$ SR2 0

\#S 4.4.6/31/11:

$02.03 .03 /$ SR18 $02.03 .03 /$ SR19

\#S $4.4 .7 / 32 / 3$ :

03.03/SR01 03.03/SR02 03.03/SR03 03.03/SR09 03.03/SR10 03.03/SR11

$03.03 /$ SR12 $03.05 /$ SR01 $03.05 /$ SR02 $03.05 /$ SR03

\#S $4.4 .7 / 32 / 4$ :

$03.01 /$ SR01 $03.01 /$ SR0 $703.03 /$ SR0 $403.05 /$ SR0 $403.05 /$ SR0 5

\#S $4.4 .7 / 32 / 5$ :

03.01/SR05 $03.01 /$ SR08 $03.01 /$ SR10 $03.01 /$ SR11 $03.04 /$ SR01

\#S $4.4 .7 / 32 / 6$ :

03.04/SR05 03.04/SR0 $03.04 /$ SR0 $703.04 /$ SR0 $803.04 / S R 1203.04 /$ SR13

03.04/SR14 $03.04 /$ SR15 $03.04 /$ SR16 $03.04 /$ SR17

\#S $4.4 .7 / 32 / 7$ :

03.02/SR02 03.02/SR03 03.03/SR06 03.03/SR07 03.03/SR08

\#S $4.4 .7 / 33 / 1$ :

03.03/SR06 03.03/SR07 03.03/SR08 03.03/SR14 03.03/SR15 03.03/SR16 $03.03 /$ SR 17

\#S $4.4 .7 / 33 / 2$ :

$03.04 /$ SR02 $03.04 /$ SR0 $303.04 /$ SR0 4

\#S $4.4 .8 / 33 / 3$ :

$02.02 .02 /$ SR 07

\#S $4.4 .9 / 33 / 4$ :

$02.02 .02 /$ SR0 4

\#S $4.5 .1 / 34 / 1$ : 


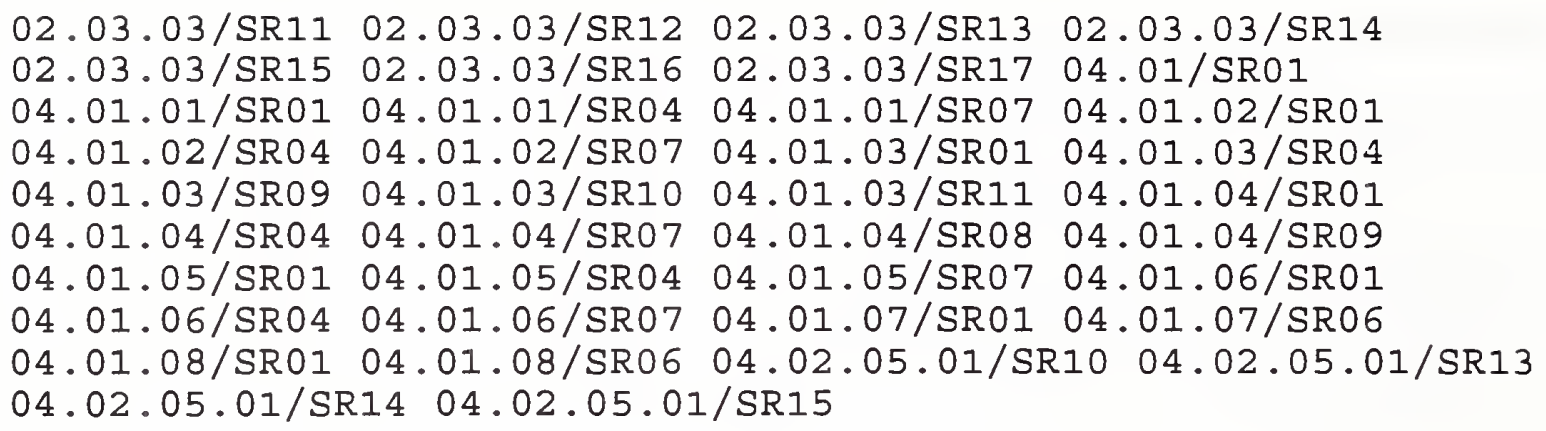


$04.02 .03 .01 / S R 27$ $04.02 .03 .01 / S R 40$ $04.02 .03 .01 / S R 47$ $04.02 .03 .01 /$ SR 64 $04.02 .03 .01 /$ SR 74 $04.02 .03 .02 /$ SR03 $04.02 .04 .01 /$ SR0 5 $04.02 .04 .01 /$ SR2 6 $04.02 .04 .01 / S R 42$ $04.02 .04 .01 /$ SR5 7 $04.02 .04 .01 / S R 70$ $04.02 .04 .01 /$ SR76 $04.02 .04 .02 /$ SR03 $04.02 .05 .01 /$ SR0 5 $04.02 .05 .01 /$ SR19 $04.02 .05 .01 /$ SR 32 $04.02 .05 .01 / S R 41$ $04.02 .05 .01 /$ SR55 $04.02 .05 .01 /$ SR 62 $04.02 .05 .01 /$ SR 68 $04.02 .05 .02 /$ SR0 4 $04.02 .05 .03 /$ SR0 3 $04.03 .04 .01 /$ SR15

$04.02 .03 .01 /$ SR3 4 $04.02 .03 .01 /$ SR 41 $04.02 .03 .01 /$ SR52 $04.02 .03 .01 /$ SR 66 $04.02 .03 .01 /$ SR79 $04.02 .03 .02 /$ SR0 4 $04.02 .04 .01 /$ SR0 6 $04.02 .04 .01 /$ SR32 $04.02 .04 .01 /$ SR4 8 $04.02 .04 .01 /$ SR 63 $04.02 .04 .01 /$ SR71 $04.02 .04 .02 /$ SR01 $04.02 .05 .01 /$ SR03 $04.02 .05 .01 /$ SR0 7 $04.02 .05 .01 / S R 26$ $04.02 .05 .01 /$ SR 34 $04.02 .05 .01 / S R 49$ $04.02 .05 .01 /$ SR 56 $04.02 .05 .01 /$ SR6 6 $04.02 .05 .01 /$ SR69 $04.02 .05 .02 /$ SRO 5 $04.02 .05 .03 /$ SR0 4
$04.02 .03 .01 /$ SR 35 $04.02 .03 .01 /$ SR 46 $04.02 .03 .01 /$ SR5 8 $04.02 .03 .01 /$ SR72 $04.02 .03 .01 /$ SR80 $04.02 .03 .02 /$ SR0 5 $04.02 .04 .01 /$ SR19 $04.02 .04 .01 /$ SR3 7 $04.02 .04 .01 /$ SR55 $04.02 .04 .01 /$ SR 65 $04.02 .04 .01 /$ SR75 $04.02 .04 .02 /$ SR0 2 $04.02 .05 .01 /$ SR0 4 $04.02 .05 .01 /$ SR18 $04.02 .05 .01 /$ SR2 7 $04.02 .05 .01 / S R 40$ $04.02 .05 .01 /$ SR50 $04.02 .05 .01 /$ SR61 $04.02 .05 .01 /$ SR 67 $04.02 .05 .02 / \mathrm{SR} 03$ $04.02 .05 .02 /$ SR0 6 $04.02 .05 .03 /$ SR05 $04.03 .01 /$ SR0 7

\#S $4.5 .2 / 38 / 7:$

$04.02 .01 .02 /$ SR0 $64.02 .01 .02 /$ SR0 $04.02 .02 .02 /$ SR0 6

$04.02 .02 .02 /$ SR0 $704.02 .03 .02 /$ SR0 $04.02 .03 .02 /$ SR0 7

$04.02 .04 .02 /$ SR0 $404.02 .04 .02 /$ SR0 $504.02 .05 .02 /$ SR0 7

$04.02 .05 .02 /$ SR08 $04.02 .05 .03 /$ SR06 $04.02 .05 .03 /$ SR0 $704.03 .01 /$ SR09

\#S $4.5 .2 / 39 / 2:$

$04.03 .01 /$ SR08 $04.03 .01 /$ SR09

\#S $4.5 .2 / 39 / 3:$

$04.03 .02 .02 /$ SR 15

\#S $4.5 .2 / 39 / 5$ :

$04.03 .01 /$ SR0 $404.03 .01 /$ SR05 $04.03 .01 /$ SR0 $64.03 .01 /$ SR 10

\#S $4.5 .2 / 39 / 6:$

$04.03 .01 /$ SR01

\#S $4.5 .2 / 39 / 7$ :

$04.02 .01 .01 /$ SR0 6

$04.02 .01 .01 /$ SR16

$04.02 .01 .02 /$ SR0 6

$04.02 .01 .02 / S R 43$

$04.02 .01 .02 /$ SR 47

$04.02 .02 .01 /$ SR0 6

$04.02 .02 .01 /$ SR16

$04.02 .02 .02 /$ SR0 6

$04.02 .02 .02 /$ SR 43

$04.02 .02 .02 /$ SR 48

$04.02 .03 .01 /$ SR2 1

$04.02 .01 .01 / \mathrm{SR} 07$

$04.02 .01 .01 / S R 21$

$04.02 .01 .02 /$ SR0 7

$04.02 .01 .02 /$ SR 44

$04.02 .01 .02 / S R 48$

$04.02 .02 .01 /$ SR0 7

$04.02 .02 .01 /$ SR2 2

$04.02 .02 .02 /$ SR0 7

$04.02 .02 .02 / S R 44$

$04.02 .02 .02 / S R 49$

$04.02 .03 .01 /$ SR2 2
$04.02 .01 .01 /$ SR15

$04.02 .01 .01 / \mathrm{SR} 22$

$04.02 .01 .02 /$ SR 35

$04.02 .01 .02 /$ SR 45

$04.02 .01 .02 / S R 49$

$04.02 .02 .01 /$ SR 15

$04.02 .02 .01 /$ SR2 3

$04.02 .02 .02 / S R 35$

$04.02 .02 .02 / S R 45$

$04.02 .02 .02 / S R 50$

$04.02 .03 .01 /$ SR2 8 
$04.02 .03 .01 /$ SR3 7

$04.02 .03 .01 /$ SR 49

$04.02 .03 .02 /$ SR3 8

$04.02 .03 .02 /$ SR 57

$04.02 .03 .02 /$ SR 65

$04.02 .04 .01 /$ SR15

$04.02 .04 .01 /$ SR 34

$04.02 .04 .02 /$ SR0 4

$04.02 .04 .02 /$ SR 12

$04.02 .04 .02 /$ SR2 7

$04.02 .04 .02 /$ SR 31

$04.02 .05 .01 /$ SR10

$04.02 .05 .01 /$ SR15

$04.02 .05 .01 /$ SR2 8

$04.02 .05 .01 /$ SR 42

$04.02 .05 .01 /$ SR52

$04.02 .05 .02 /$ SR 07

$04.02 .05 .02 /$ SR 39

$04.02 .05 .02 /$ SR5 3

$04.02 .05 .03 /$ SR 30

$04.02 .05 .03 /$ SR 47

$04.02 .05 .03 /$ SR51
$04.02 .03 .01 / S R 43$

$04.02 .03 .02 /$ SRO 6

$04.02 .03 .02 /$ SR39

$04.02 .03 .02 /$ SR 61

$04.02 .03 .02 /$ SR 66

$04.02 .04 .01 /$ SR20

$04.02 .04 .01 /$ SR3 8

$04.02 .04 .02 /$ SR0 5

$04.02 .04 .02 /$ SR 13

$04.02 .04 .02 /$ SR2 9

$04.02 .05 .01 /$ SR0 8

$04.02 .05 .01 /$ SR 13

$04.02 .05 .01 / S R 20$

$04.02 .05 .01 /$ SR29

$04.02 .05 .01 /$ SR 43

$04.02 .05 .01 /$ SR 57

$04.02 .05 .02 /$ SR0 8

$04.02 .05 .02 /$ SR 51

$04.02 .05 .03 /$ SR0 6

$04.02 .05 .03 /$ SR3 8

$04.02 .05 .03 /$ SR 48

$04.02 .05 .03 /$ SR 52
$04.02 .03 .01 / S R 48$

$04.02 .03 .02 /$ SR0 7

$04.02 .03 .02 / S R 40$

$04.02 .03 .02 /$ SR 64

$04.02 .04 .01 /$ SR14

$04.02 .04 .01 /$ SR29

$04.02 .04 .01 /$ SR39

$04.02 .04 .02 /$ SRI1

$04.02 .04 .02 /$ SR2 4

$04.02 .04 .02 /$ SR30

$04.02 .05 .01 /$ SR09

$04.02 .05 .01 /$ SR14

$04.02 .05 .01 / S R 21$

$04.02 .05 .01 /$ SR 35

$04.02 .05 .01 /$ SR51

$04.02 .05 .01 /$ SR 58

$04.02 .05 .02 /$ SR 35

$04.02 .05 .02 /$ SR52

$04.02 .05 .03 /$ SR0 7

$04.02 .05 .03 /$ SR 46

$04.02 .05 .03 /$ SR 50

$04.03 .01 /$ SR08 $04.03 .01 /$ SR0 9

\#S 4.5.2/40/1:

$04.01 /$ SR01 $04.03 .03 /$ SR01 $04.03 .03 /$ SR0 4

\#S $4.5 .2 / 40 / 3:$

$04.02 .01 .01 /$ SR03 $04.02 .01 .02 /$ SR03 $04.02 .02 .01 /$ SR0 3

$04.02 .02 .02 /$ SR03 $04.02 .03 .01 /$ SR11 $04.02 .05 .03 /$ SR03

$04.03 .01 /$ SR0 $704.03 .04 .01 /$ SRI5

\#S $4.5 .2 / 41 / 2$ :

$04.02 .04 .01 /$ SR0 5

\#S $4.5 .2 / 41 / 3$ :

$04.02 .05 .01 /$ SR03

\#S $4.5 .2 / 41 / 4$ :

$04.02 .05 .01 /$ SR0 4

\#S $4.5 .2 / 42 / 2:$

$04.03 .01 /$ SR0 $84.03 .01 /$ SR09

\#S $4.5 .2 / 42 / 3:$

$04.02 .01 .02 /$ SR 10

$04.02 .01 .02 / \mathrm{SR} 24$

$04.02 .02 .02 /$ SRI1

$04.02 .02 .02 /$ SR2 5

$04.02 .03 .02 /$ SR 22

$04.02 .04 .02 /$ SR09

$04.02 .05 .02 /$ SR2 3

$04.02 .01 .02 /$ SR11

$04.02 .01 .02 / \mathrm{SR} 25$

$04.02 .01 .02 /$ SR 22

$04.02 .02 .02 / \mathrm{SR} 22$

$04.02 .02 .02 /$ SR10

$04.02 .03 .02 /$ SR10

$04.02 .02 .02 / S R 24$

$04.02 .03 .02 /$ SR2 4

$04.02 .03 .02 /$ SRI1

$04.02 .05 .03 /$ SR 10

$04.02 .05 .02 /$ SR11

$04.02 .05 .02 / S R 12$

$04.02 .05 .02 / S R 25$

$04.02 .05 .02 / S R 26$

$04.02 .05 .03 /$ SR2 5

$04.02 .05 .03 /$ SR22

$04.02 .05 .03 /$ SR2 4

$04.02 .05 .04 /$ SR1 7

$04.02 .05 .04 /$ SRO 2

$04.02 .05 .04 /$ SR 16

$04.03 .02 .01 /$ SR0 2

$04.03 .02 .02 /$ SRI2 
$04.03 .02 .02 /$ SR13 $04.03 .02 .02 /$ SR14

\#S $4.5 .2 / 43 / 2$ :

$04.02 .01 .02 /$ SR10 $04.02 .01 .02 /$ SR11 $04.02 .02 .02 /$ SR10 $04.02 .02 .02 /$ SR11 $04.02 .03 .02 /$ SR10 $04.02 .05 .02 / S R 11$ $04.02 .05 .03 /$ SR10 $04.03 .02 .01 /$ SR02

\#S $4.5 .2 / 43 / 3:$

$04.02 .01 .01 /$ SR0 7 $04.02 .01 .02 / \mathrm{SR} 48$ $04.02 .02 .01 / \mathrm{SR} 23$ $04.02 .02 .02 /$ SR 50 $04.02 .03 .02 /$ SRO 7 $04.02 .04 .01 /$ SR39 $04.02 .04 .02 /$ SR31 $04.02 .05 .01 /$ SR58 $04.02 .05 .03 /$ SR0 7 $04.02 .01 .01 /$ SR22 $04.02 .01 .02 /$ SR 49 $04.02 .02 .02 /$ SR0 7 $04.02 .03 .01 /$ SR2 2 $04.02 .03 .02 /$ SR6 6 $04.02 .04 .01 /$ SR79 $04.02 .05 .01 /$ SR21 $04.02 .05 .02 /$ SR0 8 $04.02 .05 .03 /$ SR51

$04.02 .01 .02 /$ SR0 7 $04.02 .02 .01 /$ SR0 7 $04.02 .02 .02 /$ SR 49 $04.02 .03 .01 /$ SR 49 $04.02 .04 .01 /$ SR 15 $04.02 .04 .02 /$ SR0 5 $04.02 .05 .01 /$ SR29 $04.02 .05 .02 /$ SR 53 $04.02 .05 .03 /$ SR 52

\#S $4.5 .3 / 43 / 4$ :

$04.02 .01 .02 /$ SR0 $64.02 .01 .02 /$ SR0 $704.02 .01 .02 /$ SR3 5 $04.02 .01 .02 / S R 4304.02 .01 .02 / S R 4404.02 .01 .02 / S R 45$ $04.02 .01 .02 /$ SR $4704.02 .01 .02 /$ SR $48 \quad 04.02 .01 .02 / S R 49$

\#S $4.5 .3 / 43 / 5:$

$04.02 .01 .01 /$ SR0 $64.02 .01 .01 /$ SR08 $04.02 .01 .01 /$ SR0 9 $04.02 .01 .01 /$ SR10 $04.02 .01 .02 /$ SR30 $04.02 .01 .02 /$ SR 34 $04.02 .01 .02 /$ SR36 $04.02 .01 .02 /$ SR $3704.02 .01 .02 /$ SR 38

\#S $4.5 .3 / 43 / 6$ :

$04.02 .01 .01 /$ SR15 $04.02 .01 .01 /$ SR16 $04.02 .01 .02 /$ SR 44 $04.02 .01 .02 / \mathrm{SR} 45$

\#S $4.5 .4 / 43 / 7:$

$04.02 .02 .02 /$ SR0 $604.02 .02 .02 /$ SR0 $704.02 .02 .02 / S R 35$

$04.02 .02 .02 /$ SR $4304.02 .02 .02 / S R 44 \quad 04.02 .02 .02 / S R 45$

$04.02 .02 .02 /$ SR $4804.02 .02 .02 /$ SR $4904.02 .02 .02 / S R 50$

\#S $4.5 .4 / 44 / 2:$

$04.02 .02 .01 /$ SR0 $604.02 .02 .01 /$ SR0 $0404.02 .02 .01 /$ SR0 9

$04.02 .02 .01 /$ SR10 $04.02 .02 .02 /$ SR30 $04.02 .02 .02 /$ SR3 4

$04.02 .02 .02 /$ SR3 $64.02 .02 .02 /$ SR3 $704.02 .02 .02 /$ SR3 8

\#S $4.5 .4 / 44 / 3:$

$04.02 .02 .01 /$ SR15 $04.02 .02 .01 /$ SR16 $04.02 .02 .01 /$ SR17

$04.02 .02 .02 /$ SR $4404.02 .02 .02 /$ SR $4504.02 .02 .02 /$ SR 46

\#S $4.5 .5 / 44 / 6:$

$04.02 .03 .01 /$ SR01 $04.02 .03 .01 /$ SR02

\#S $4.5 .5 / 44 / 7$ :

$04.02 .03 .01 /$ SR01 $04.02 .03 .01 /$ SR02

\#S $4.5 .5 / 44 / 9$ :

$04.02 .03 .01 /$ SR60 $04.02 .03 .03 /$ SR09 $04.02 .04 .01 /$ SR50 
\#S $4.5 .5 / 44 / 10:$

$04.02 .03 .01 /$ SR21 $04.02 .03 .01 /$ SR22 $04.02 .03 .01 /$ SR3 $704.02 .03 .01 /$ SR 43 $04.02 .03 .01 /$ SR49 $04.02 .03 .02 /$ SR0 6 $04.02 .03 .02 /$ SR 38 $04.02 .03 .02 /$ SR 57 $04.02 .03 .02 /$ SR 65 $04.02 .04 .01 /$ SR 15 $04.02 .04 .01 /$ SR 34 $04.02 .04 .02 / \mathrm{SR} 04$ $04.02 .04 .02 /$ SR 12 $04.02 .04 .02 /$ SR2 7 $04.02 .04 .02 /$ SR 31

\#S $4.5 .5 / 45 / 2$ :

$04.02 .03 .01 /$ SR 36 $04.02 .03 .01 /$ SR61 $04.02 .03 .01 /$ SR 68 $04.02 .03 .02 /$ SR 58 $04.02 .03 .03 /$ SR0 8 $04.02 .04 .01 /$ SR2 7 $04.02 .04 .01 /$ SR52 $04.02 .04 .01 /$ SR59 $04.02 .04 .02 / S R 25$

\#S $4.5 .5 / 45 / 3$ : $04.02 .03 .01 /$ SR 42 $04.02 .04 .01 /$ SR33

\#S $4.5 .5 / 45 / 4$ :

$04.02 .03 .01 /$ SR 36 $04.02 .03 .03 /$ SR0 6 $04.02 .03 .03 /$ SR16 $04.02 .04 .02 /$ SR2 5
$04.02 .03 .01 /$ SR59 $04.02 .03 .01 /$ SR 65 $04.02 .03 .01 /$ SR 69 $04.02 .03 .03 /$ SR0 1 $04.02 .03 .03 /$ SR09 $04.02 .04 .01 /$ SR 49 $04.02 .04 .01 /$ SR5 6 $04.02 .04 .01 /$ SR 60 $04.02 .03 .01 /$ SR60 $04.02 .03 .01 /$ SR 67 $04.02 .03 .01 /$ SR81 $04.02 .03 .03 /$ SR0 6 $04.02 .03 .03 /$ SR10 $04.02 .04 .01 /$ SR50 $04.02 .04 .01 /$ SR58 $04.02 .04 .01 / \mathrm{SR} 72$

$04.02 .03 .02 /$ SR 62 $04.02 .04 .02 / \mathrm{SR} 28$

$04.02 .03 .01 /$ SR2 8 $04.02 .03 .01 /$ SR 48 $04.02 .03 .02 /$ SR0 7 $04.02 .03 .02 / S R 40$ $04.02 .03 .02 /$ SR 64 $04.02 .04 .01 /$ SR14 $04.02 .04 .01 /$ SR29 $04.02 .04 .01 /$ SR 39 $04.02 .04 .02 /$ SR11 $04.02 .04 .02 / S R 24$ $04.02 .04 .02 /$ SR 30

$04.02 .03 .01 /$ SR75 $04.02 .03 .02 /$ SR58 $04.02 .03 .03 /$ SR11 $04.02 .03 .03 /$ SR15 $04.02 .04 .01 /$ SR2 $704.02 .04 .01 /$ SR6 6

\#S $4.5 .5 / 45 / 5$ :

$04.02 .03 .01 /$ SR81 $04.02 .04 .01 /$ SR72

\#S $4.5 .5 / 45 / 6:$

$04.02 .03 .01 /$ SR73 $04.02 .03 .01 /$ SR75 $04.02 .03 .03 /$ SR 11 $04.02 .04 .01 /$ SR $6404.02 .04 .01 /$ SR 66

\#S $4.5 .5 / 46 / 1$ :

$04.02 .03 .01 /$ SR59 $04.02 .03 .01 /$ SR73 $04.02 .03 .01 /$ SR75 $04.02 .03 .03 /$ SR0 $804.02 .03 .03 /$ SR11 $04.02 .04 .01 /$ SR 49 $04.02 .04 .01 /$ SR6 $404.02 .04 .01 /$ SR 66

\#S $4.5 .5 / 46 / 3:$

$04.02 .03 .01 /$ SR76 $04.02 .03 .03 /$ SR $12 \quad 04.02 .04 .01 /$ SR 67

\#S $4.5 .5 / 47 / 1$ :

$04.02 .03 .01 /$ SR76 $04.02 .03 .03 /$ SR12 $04.02 .04 .01 /$ SR67 
\#S $4.5 .5 / 47 / 2$ :

$04.02 .03 .01 /$ SR 62

$04.02 .03 .01 /$ SR 82

$04.02 .03 .01 /$ SR70

$04.02 .03 .01 /$ SR 77

$04.02 .04 .01 /$ SR 68

$04.02 .04 .01 /$ SR53

$04.02 .04 .01 /$ SR61

\#S $4.5 .5 / 47 / 4:$

$04.01 .03 /$ SR0 $804.02 .03 .01 /$ SR14 $04.02 .03 .01 /$ SR15 $04.02 .03 .01 /$ SR20

$04.02 .03 .01 /$ SR21 $04.02 .03 .02 /$ SR30 $04.02 .03 .02 /$ SR 31

$04.02 .03 .02 / \mathrm{SR} 40$

$04.02 .03 .02 / \mathrm{SR} 46$

$04.02 .04 .01 /$ SR13

$04.02 .03 .02 / S R 4104.02 .03 .02 / S R 42$

$04.02 .04 .01 /$ SR0 $04.02 .04 .01 /$ SR0 8

$04.02 .04 .02 /$ SR14

$04.02 .04 .01 /$ SR14 $04.02 .04 .02 /$ SR13

$04.02 .04 .02 /$ SR15 $04.02 .04 .02 /$ SR19

\#S $4.5 .5 / 47 / 5:$

$04.02 .03 .01 /$ SR2 $64.02 .03 .01 /$ SR29 $04.02 .03 .01 /$ SR30

$04.02 .03 .01 /$ SR3 $04.02 .03 .01 /$ SR61 $04.02 .03 .02 /$ SR3 4

$04.02 .03 .02 /$ SR4 $804.02 .03 .02 /$ SR49 $04.02 .03 .02 /$ SR59

$04.02 .04 .01 /$ SR18 $04.02 .04 .01 /$ SR21 $04.02 .04 .01 /$ SR2 2

$04.02 .04 .01 /$ SR29 $04.02 .04 .01 /$ SR52 $04.02 .04 .02 /$ SR 10

$04.02 .04 .02 /$ SR2 $104.02 .04 .02 /$ SR2 $204.02 .04 .02 / S R 26$

\#S $4.5 .5 / 48 / 1$ :

$04.02 .03 .01 /$ SR31 $04.02 .03 .02 /$ SR50 $04.02 .03 .03 /$ SR 14

$04.02 .04 .01 /$ SR23 $04.02 .04 .02 /$ SR23

\#S $4.5 .5 / 48 / 3:$

$04.02 .03 .01 / \mathrm{SR} 03$

$04.02 .03 .01 /$ SRO 7

$04.02 .03 .01 /$ SR0 4

$04.02 .03 .01 /$ SR0 6

$04.02 .03 .02 / S R 48$

$04.02 .03 .01 /$ SR29

$04.02 .03 .01 /$ SR 30

$04.02 .04 .02 /$ SR 22

$04.02 .04 .02 /$ SR2 1

\#S $4.5 .5 / 48 / 4:$

$04.02 .03 .01 /$ SR0 $504.02 .03 .01 /$ SR08

\#S $4.5 .5 / 48 / 5:$

$04.02 .03 .01 /$ SR14 $04.02 .03 .01 /$ SR15

$04.02 .03 .01 /$ SR19

$04.02 .03 .02 /$ SR 36

$04.02 .03 .01 /$ SR2 8

$04.02 .03 .02 /$ SR 41

$04.02 .03 .02 /$ SR 47

$04.02 .03 .02 / S R 45$

$04.02 .04 .01 /$ SR0 7

$04.02 .04 .01 /$ SR0 8

$04.02 .04 .01 /$ SR2 0

$04.02 .03 .01 /$ SR 18

$04.02 .03 .02 /$ SR 31

$04.02 .03 .02 /$ SR 42

$04.02 .03 .03 /$ SR0 4

$04.02 .04 .01 /$ SRI2

$04.02 .04 .01 /$ SR11

$04.02 .04 .02 /$ SR15

$04.02 .04 .02 /$ SR1 8

$04.02 .04 .02 /$ SR14

$04.02 .04 .02 / S R 20$

\#S $4.5 .5 / 48 / 6$ :

$04.02 .03 .01 /$ SR 16

$04.02 .03 .02 /$ SR 40

$04.02 .03 .03 /$ SR02

$04.02 .03 .01 /$ SR 17

$04.02 .03 .01 / S R 21$

$04.02 .03 .02 /$ SR 43

$04.02 .03 .02 / S R 44$

$04.02 .04 .01 /$ SR09

$04.02 .03 .03 /$ SR0 3

$04.02 .03 .03 /$ SR0 4

$04.02 .04 .02 /$ SR13

$04.02 .04 .01 /$ SR 10

$04.02 .04 .01 /$ SR14

$04.02 .04 .02 /$ SR16 $04.02 .04 .02 /$ SR 17

\#S $4.5 .5 / 48 / 7$ :

$04.02 .03 .01 /$ SR03 $04.02 .03 .01 /$ SR0 $404.02 .03 .01 /$ SR0 6

$04.02 .03 .01 /$ SR0 $704.02 .03 .01 /$ SR16 $04.02 .03 .01 /$ SR59

$04.02 .03 .02 /$ SR43 $04.02 .03 .03 /$ SR02 $04.02 .03 .03 /$ SR0 8 


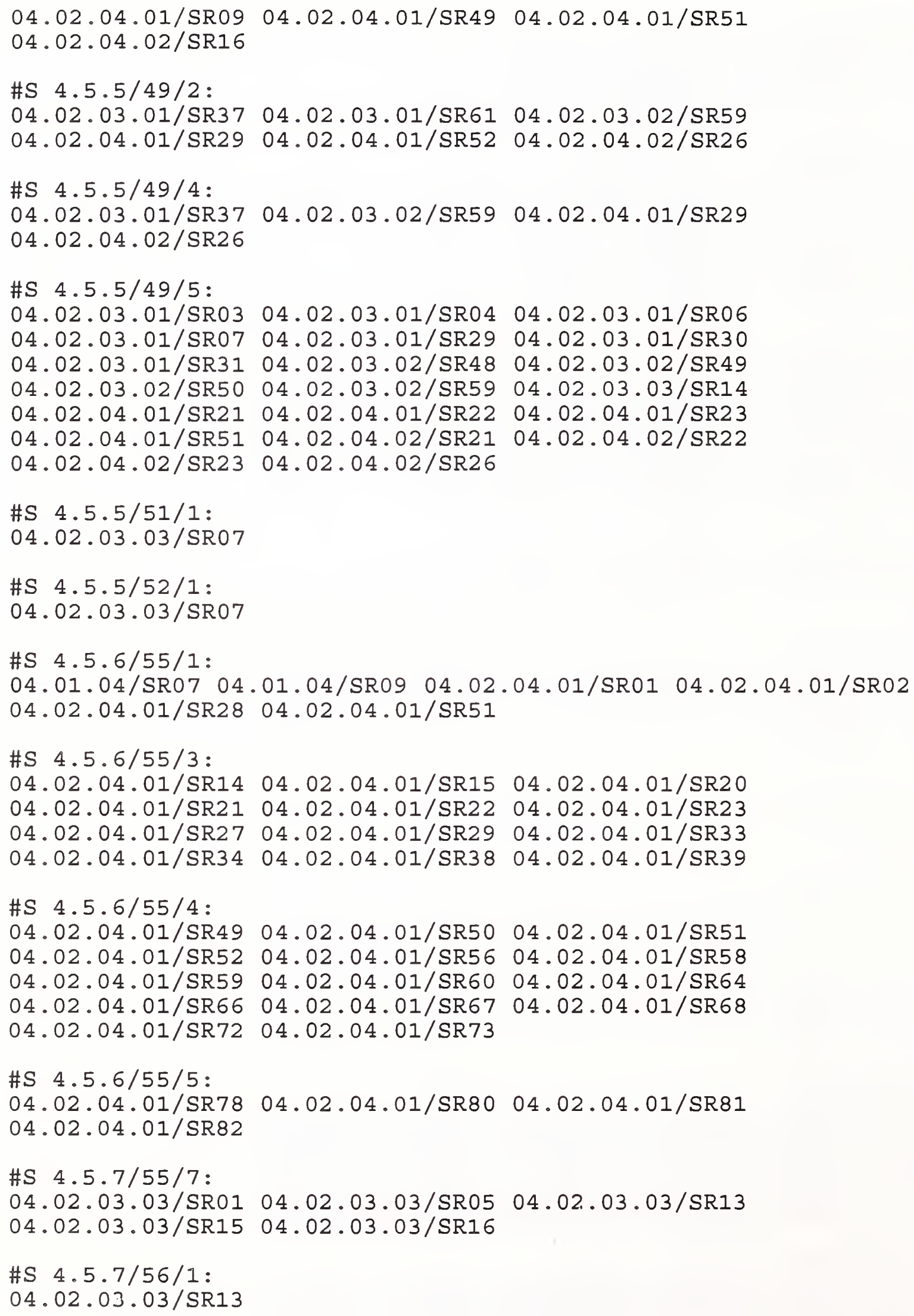


\#S $4.5 .7 / 56 / 2$ :

$04.02 .03 .03 /$ SR15 $04.02 .03 .03 /$ SR16

\#S $4.5 .7 / 56 / 3$ :

$04.02 .03 .03 /$ SR17 $04.02 .03 .03 /$ SR18

\#S $4.5 .7 / 56 / 4:$

$04.02 .03 .03 /$ SR17

\#S $4.5 .7 / 57 / 1$ :

$04.02 .03 .03 /$ SR07 $04.02 .03 .03 /$ SRI7 $04.02 .03 .03 /$ SR18

\#S $4.5 .8 / 58 / 2:$

$04.02 .05 .02 /$ SR0 $704.02 .05 .02 /$ SR0 $804.02 .05 .02 /$ SR 35

$04.02 .05 .02 /$ SR39 $04.02 .05 .02 /$ SR51 $04.02 .05 .02 /$ SR 52

$04.02 .05 .02 /$ SR5 3

\#S $4.5 .8 / 58 / 3:$

$04.02 .05 .01 /$ SR0 $604.02 .05 .01 /$ SR0 $804.02 .05 .01 /$ SR 10

$04.02 .05 .01 /$ SR11 $04.02 .05 .01 /$ SR12 $04.02 .05 .01 /$ SR13

$04.02 .05 .01 /$ SR14 $04.02 .05 .01 /$ SR15 $04.02 .05 .01 /$ SR2 0

$04.02 .05 .01 /$ SR22 $04.02 .05 .01 /$ SR23 $04.02 .05 .02 /$ SR 31

$04.02 .05 .02 /$ SR 36

\#S $4.5 .8 / 59 / 1$ :

$04.02 .05 .01 /$ SR71 $04.02 .05 .01 /$ SR72 $04.02 .05 .01 /$ SR73

$04.02 .05 .01 /$ SR75 $04.02 .05 .01 /$ SR76

\#S $4.5 .8 / 60 / 1$ :

$04.02 .05 .01 /$ SR71 $04.02 .05 .01 /$ SR74

\#S $4.5 .8 / 60 / 2:$

$04.02 .05 .01 /$ SR71 $04.02 .05 .01 /$ SR75 $04.02 .05 .01 / S R 76$

$04.02 .05 .01 /$ SR77 $04.02 .05 .01 /$ SR78 $04.02 .05 .01 /$ SR79

\#S $4.5 .8 / 61 / 2:$

$04.02 .05 .02 /$ SR $4704.02 .05 .02 /$ SR4 8

\#S $4.5 .8 / 61 / 3:$

$04.02 .05 .01 /$ SR20 $04.02 .05 .01 /$ SR2 2

\#S $4.5 .8 / 62 / 1$ :

$04.02 .05 .01 /$ SR23 $04.02 .05 .02 /$ SR 49

\#S $4.5 .8 / 62 / 2$ :

$04.02 .05 .02 /$ SR 32

\#S $4.5 .9 / 62 / 4:$

$04.02 .05 .03 /$ SR11

\#S $4.5 .9 / 62 / 5:$

$04.02 .05 .01 /$ SR33 $04.02 .05 .01 /$ SR35 $04.02 .05 .01 /$ SR 36

$04.02 .05 .03 /$ SR0 $64.02 .05 .03 /$ SR07 $04.02 .05 .03 /$ SR29

$04.02 .05 .03 /$ SR30 $04.02 .05 .03 /$ SR31 $04.02 .05 .03 /$ SR 38 
$04.02 .05 .03 / S R 4604.02 .05 .03 / S R 4704.02 .05 .03 / S R 48$ $04.02 .05 .03 /$ SR50 $04.02 .05 .03 /$ SR51 04.02.05.03/SR52

\#S $4.5 .9 / 62 / 6:$

$04.02 .05 .01 /$ SR3 7

\#S $4.5 .9 / 62 / 7$ :

$04.02 .05 .01 /$ SR 42

$04.02 .05 .01 / \mathrm{SR} 45$

$04.02 .05 .03 /$ SR3 7

$04.02 .05 .01 /$ SR4 $304.02 .05 .01 / S R 44$

$04.02 .05 .01 /$ SR $4604.02 .05 .03 /$ SR3 3

$04.02 .05 .03 /$ SR 41

$04.02 .05 .03 /$ SR39 $04.02 .05 .03 /$ SR40

\#S $4.5 .9 / 62 / 8:$

$04.02 .05 .01 /$ SR51 $04.02 .05 .01 /$ SR52 $04.02 .05 .03 /$ SR 47

$04.02 .05 .03 / \mathrm{SR} 48$

\#S $4.5 .9 / 62 / 9:$

$04.02 .05 .01 / \operatorname{SR} 37$

\#S $4.5 .12 / 63 / 4:$

$04.02 .01 .01 /$ SR21 $04.02 .01 .02 /$ SR $4804.02 .01 .02 / S R 49$

$04.02 .02 .01 /$ SR22 $04.02 .02 .02 /$ SR $4904.02 .02 .02 /$ SR50

$04.02 .03 .01 /$ SR $4804.02 .03 .02 /$ SR65 $04.02 .03 .02 /$ SR 66

$04.02 .04 .01 /$ SR3 $84.02 .04 .02 /$ SR30 $04.02 .04 .02 /$ SR3 1

$04.02 .05 .01 /$ SR28 $04.02 .05 .01 /$ SR5 $704.02 .05 .02 /$ SR5 2

$04.02 .05 .02 /$ SR53 $04.02 .05 .03 /$ SR51 $04.02 .05 .03 /$ SR52

\#S $4.5 .12 / 63 / 5:$

$04.03 .02 .01 /$ SR0 $804.03 .02 .02 /$ SR10 $04.03 .02 .02 /$ SR11

$04.03 .02 .02 /$ SR $1204.03 .02 .02 /$ SR $30 \quad 04.03 .02 .02 /$ SR 31

\#S $4.5 .12 / 63 / 6:$

$04.03 .02 .02 / \mathrm{SR} 20$

\#S $4.5 .12 / 63 / 7:$

$04.03 .02 .01 /$ SR01 $04.03 .02 .01 /$ SR02 $04.03 .02 .01 /$ SRO 4

$04.03 .02 .01 /$ SR0 $54.03 .02 .02 / S R 2704.03 .02 .02 / S R 32$

\#S $4.5 .12 / 63 / 8$ :

$04.03 .02 .01 /$ SR02 $04.03 .02 .01 /$ SR0 $504.03 .02 .01 /$ SR09

$04.03 .02 .01 /$ SR10 $04.03 .02 .02 /$ SR21 $04.03 .02 .02 /$ SR 27

$04.03 .02 .02 /$ SR3 2

\#S $4.5 .12 / 64 / 1$ :

$04.03 .02 .02 /$ SR16 $04.03 .02 .02 /$ SR17 $04.03 .02 .02 /$ SR18

$04.03 .02 .02 /$ SR19 $04.03 .02 .02 /$ SR22 $04.03 .02 .02 /$ SR2 3

$04.03 .02 .02 /$ SR2 $404.03 .02 .02 /$ SR2 $504.03 .02 .02 / S R 26$

$04.03 .02 .02 /$ SR2 $804.03 .02 .02 /$ SR2 9

\#S $4.5 .12 / 64 / 2$ :

$04.03 .02 .02 /$ SR3 3

\#S $4.5 .12 / 64 / 3:$

$04.03 .02 .02 /$ SR0 $404.03 .02 .02 /$ SR0 $504.03 .02 .02 /$ SR0 6 
\#S $4.5 .13 / 64 / 4:$

$04.01 /$ SRO1

\#S $4.5 .15 / 64 / 7:$

$04.03 .04 .01 /$ SR01 $04.03 .04 .01 /$ SR0 $404.03 .04 .01 /$ SR12

$04.03 .04 .01 /$ SR13

\#S $4.5 .15 / 64 / 8:$

$04.03 .04 .02 /$ SR01 $04.03 .04 .02 /$ SR0 $404.03 .04 .02 /$ SR0 5

$04.03 .04 .02 /$ SR0 8

\#S $4.5 .15 / 65 / 2$ :

$04.03 .04 .01 /$ SR12 $04.03 .04 .01 /$ SR13

\#S $4.5 .15 / 65 / 4:$

$04.03 .04 .01 /$ SR0 $704.03 .04 .01 /$ SR0 $04.03 .04 .01 /$ SR09

$04.03 .04 .02 /$ SR10 $04.03 .04 .02 /$ SR11

\#S $4.5 .16 / 65 / 5$ :

$04.01 .07 /$ SR14 $04.02 .03 .02 /$ SR31

\#S $4.6 .1 / 67 / 2$ :

$04.01 /$ SR02 $05.01 .01 /$ SR08 $05.01 .02 /$ SR01

\#S $4.6 .1 / 67 / 3$ :

$04.01 /$ SRO2

\#S 4.6.1/68/1:

$05.01 .02 /$ SR02

\#S $4.6 .2 / 68 / 7$ :

05.01.01/SR06 05.01.01/SR08 05.01.02/SR01 05.01.02/SR06

\#S $4.6 .3 / 69 / 2:$

$04.03 .03 /$ SR0 $04.03 .03 /$ SR0 $05.02 /$ SR0 $05.02 /$ SR0 7

\#S $4.6 .3 / 69 / 3:$

$05.02 /$ SR19 $05.02 / S R 2005.02 / S R 21$

\#S $4.6 .3 / 70 / 1$ :

$05.02 /$ SR0 3

\#S $4.6 .3 / 70 / 2$ :

$05.02 /$ SR11 $05.02 /$ SR12 $05.02 /$ SR13 $05.02 /$ SR14 $05.02 /$ SR15

\#S $4.6 .3 / 70 / 3:$

$05.02 /$ SR0 4

\#S $4.6 .3 / 70 / 4:$

$05.02 /$ SR01

\#S $4.6 .3 / 70 / 5$ :

$05.02 /$ SR22 
\#S $4.6 .3 / 70 / 6$ :

$05.02 /$ SR2 9

\#S $4.6 .3 / 70 / 7:$

$05.02 /$ SR $3005.02 /$ SR $3105.02 /$ SR 32

\#S $4.6 .3 / 70 / 9:$

$04.03 .03 /$ SR0 $64.03 .03 /$ SR0 $0505.02 /$ SR16 $05.02 /$ SR17 $05.02 /$ SR2 3

05.02 /SR2 $405.02 /$ SR2 $505.02 /$ SR2 $605.02 /$ SR2 $705.02 /$ SR2 8

\#S $4.6 .3 / 71 / 1$ :

$04.03 .03 /$ SR0 $64.03 .03 /$ SR0 $05.02 /$ SR02 $05.02 /$ SR2 $305.02 /$ SR2 5

05.02 /SR2 $65.02 /$ SR2 $705.02 /$ SR2 8

\#S $4.6 .3 / 72 / 1$ :

$05.02 /$ SR16 $05.02 /$ SR17 $05.02 /$ SR2 $205.02 /$ SR2 $305.02 /$ SR2 $405.02 / S R 25$

$05.02 /$ SR2 $605.02 /$ SR2 $705.02 /$ SR2 8 05.02/SR29 $05.02 /$ SR31 05.02/SR32

\#S $4.6 .3 / 73 / 1$ :

$05.02 /$ SR16 $05.02 /$ SR17 $05.02 /$ SR22 $05.02 /$ SR23 $05.02 /$ SR24 $05.02 /$ SR2 5

$05.02 /$ SR2 $605.02 /$ SR2 $705.02 /$ SR2 $805.02 /$ SR2 $905.02 /$ SR $3105.02 /$ SR 32

\#S $4.6 .3 / 74 / 1:$

$05.02 /$ SR19

\#S $4.6 .3 / 75 / 1$ :

$05.02 /$ SR19

\#S $4.6 .3 / 75 / 3:$

$05.02 /$ SR2 3

\#S $4.6 .6 / 76 / 3$ :

$04.03 .03 /$ SR01 $04.03 .03 /$ SR04 04.03.03/SR09 $04.03 .03 /$ SR10

\#S $4.6 .6 / 76 / 4$ :

$04.03 .04 .02 /$ SR09

\#S $4.7 .2 / 77 / 3:$

$04.01 /$ SR01 06.01.01/SR01 06.01.01/SR02 06.01.02/SR16

$06.01 .02 /$ SR17 $06.02 .01 /$ SR01 $06.02 .01 /$ SR02

\#S $4.7 .2 / 77 / 4:$

$06.01 .02 /$ SR2 0

\#S $4.7 .2 / 77 / 6$ :

$06.01 .02 /$ SR2 1

\#S $4.7 .2 / 77 / 7$ :

$06.01 .02 /$ SR01 $06.01 .02 /$ SR04 06.01.02/SR07 06.01.02/SR08

\#S $4.7 .2 / 78 / 2$ :

$06.01 .02 /$ SR09 $06.01 .02 /$ SR12 $06.01 .02 /$ SR15 
\#S $4.7 .2 / 78 / 3:$

$06.01 .02 /$ SR 42

\#S $\quad 4.7 .2 / 78 / 4:$

$06.01 .02 /$ SR2 $406.01 .02 /$ SR2 $706.01 .02 /$ SR $4506.01 .02 /$ SR 46 $06.01 .02 /$ SR 48

\#S $4.7 .2 / 78 / 5:$

$06.01 .02 /$ SR 47

\#S $4.7 .2 / 78 / 6:$

$06.01 .02 / \operatorname{SR} 46$

\#S $4.7 .2 / 78 / 7:$

$06.01 .02 /$ SR 48

\#S $4.7 .2 / 79 / 1$ :

$06.01 .02 /$ SR 38

\#S $4.7 .2 / 79 / 2:$

$06.01 .02 /$ SR33 $06.01 .02 /$ SR 49

\#S $4.7 .2 / 79 / 3$ :

$06.01 .02 /$ SR30 $06.01 .02 /$ SR3 6

\#S $4.7 .3 / 79 / 4:$

06.01.01/SR03 06.01.01/SR04 06.01.01/SR05 $06.01 .01 /$ SR06

$06.01 .01 /$ SR07 06.01.01/SR08 06.01.01/SR09 $06.01 .01 /$ SR10

$06.01 .01 /$ SR11 $06.01 .01 /$ SR12 $06.01 .01 /$ SR13 $06.01 .01 /$ SR14

$06.01 .01 /$ SR15 $06.01 .01 /$ SR16 $06.01 .01 /$ SR17 $06.01 .01 /$ SR18

\#S $4.7 .3 / 80 / 1$ :

$02.03 .03 /$ SR10 $04.01 /$ SR01

\#S $\quad 4.7 .4 / 82 / 1:$

$06.02 .01 /$ SR01

\#S $4.7 .4 / 83 / 3$ :

$06.02 .02 /$ SR0 8

\#S $4.7 .4 / 83 / 4:$

$06.02 .02 /$ SR0 7

\#S $4.7 .4 / 83 / 6:$

$06.02 .02 /$ SR09 $06.02 .02 /$ SR10 $06.02 .02 /$ SRI1 $06.02 .02 /$ SR14

$06.02 .02 /$ SR 15

\#S $4.7 .4 / 83 / 7$ :

$06.02 .02 /$ SR 16

\#S $4.7 .4 / 84 / 1$ :

$06.02 .02 /$ SR2 3

\#S $4.7 .4 / 84 / 2:$ 
$06.02 .02 /$ SR2 $506.02 .02 /$ SR2 $606.02 .02 /$ SR2 7

\#S $4.7 .4 / 84 / 3:$

$06.02 .02 /$ SR2 9

\#S $4.7 .4 / 84 / 4:$

$06.02 .02 /$ SR2 8

\#S $4.7 .4 / 84 / 5$ :

$06.02 .02 /$ SR19 $06.02 .02 /$ SR20

\#S $4.7 .4 / 84 / 6:$

$06.02 .02 /$ SRO1

\#S $4.7 .5 / 84 / 7:$

$06.02 .01 /$ SR01

\#S $4.7 .5 / 84 / 8$ :

$06.02 .01 /$ SR01

\#S $4.7 .5 / 84 / 9$ :

$06.02 .01 /$ SR03

\#S $4.7 .5 / 84 / 10:$

$06.02 .01 /$ SR0 4

\#S $4.7 .5 / 85 / 3:$

$06.02 .01 /$ SR0 5

\#S $4.7 .5 / 85 / 4:$

$06.02 .01 /$ SR0 6

\#S $4.7 .5 / 86 / 4:$

$06.02 .01 /$ SR0 7

\#S $4.7 .5 / 86 / 5$ :

$06.02 .01 /$ SR09

\#S $4.7 .5 / 86 / 6$ :

$06.02 .01 /$ SR0 8

\#S $4.7 .5 / 86 / 9$ :

$06.02 .01 /$ SR 10

\#S $4.7 .5 / 89 / 1$ :

$06.02 .01 /$ SR11

\#S $4.7 .5 / 89 / 2$ :

$06.02 .01 /$ SR13

\#S $4.7 .5 / 89 / 3$ :

$06.02 .01 /$ SR12

\#S $4.7 .6 / 90 / 5$ : 
$04.01 /$ SR01 $06.03 /$ SR01

\#S $4.7 .6 / 90 / 6$ :

$04.02 .04 .01 /$ SR2 $804.02 .04 .01 /$ SR51 $06.03 /$ SR0 4

\#S $4.7 .6 / 91 / 2$ :

$05.02 /$ SR10 $06.03 /$ SR01 $06.03 /$ SR0 $806.03 /$ SR11

\#S $4.7 .6 / 91 / 3$ :

06.03/SR06 06.03/SR07 06.03/SR09 06.03/SR10

\#S $4.7 .6 / 91 / 4$ :

$06.03 /$ SR14 $06.03 /$ SR15

\#S 4.7.6/91/5:

$06.03 /$ SR16 $06.03 /$ SR18

\#S $4.7 .6 / 91 / 6$ :

$06.03 /$ SR2 0

\#S $4.7 .6 / 91 / 7$ :

$06.03 /$ SR1 $706.03 /$ SR19

\#S $4.7 .6 / 91 / 9$ :

$04.02 .04 .01 /$ SR2 $804.02 .04 .01 /$ SR51

\#S $4.7 .6 / 92 / 4:$

$06.03 /$ SR0 5

\#S $4.10 / 107 / 2$ :

01/SR01 01/SR02

\#S $4.10 / 107 / 3$ :

05.01.01/SR03 05.01.01/SR04 05.01.01/SR05

\#S $4.10 / 107 / 4$ :

$02.02 .01 /$ SR09

\#S $4.10 / 107 / 6$ :

$02.02 .01 /$ SR09 $05.01 .01 /$ SR02

\#S $4.10 / 107 / 8$ :

$02.01 .01 /$ SR08

\#S 4.11/108/1:

$04.02 .01 .02 /$ SR14 $04.02 .01 .02 /$ SR15 $04.02 .02 .02 /$ SR14

$04.02 .02 .02 /$ SR15 $04.02 .03 .02 /$ SR14 $04.02 .03 .02 /$ SR15

$04.02 .05 .02 /$ SR15 $04.02 .05 .02 /$ SR16 $04.02 .05 .03 /$ SR14

$04.02 .05 .03 /$ SR15 $04.02 .05 .04 /$ SR05 $04.02 .05 .04 /$ SR0 6

$04.03 .02 .01 /$ SR0 $504.03 .02 .02 /$ SR32 $04.03 .03 /$ SR0 5

\#S $4.14 / 113 / 2:$

$04.02 .03 .02 /$ SR 30 
\#S $4.14 / 113 / 3:$

$04.02 .01 .02 /$ SR2 1

$04.02 .02 .02 /$ SR2 1

$04.02 .03 .01 /$ SR18

$04.02 .03 .02 /$ SR 45

$04.02 .04 .01 /$ SR7 7

$04.02 .05 .02 /$ SR2 3

$04.02 .05 .03 /$ SR2 2

$06.01 .02 /$ SR39
$04.02 .01 .02 / S R 2204.02 .01 .02 / S R 30$

$04.02 .02 .02 /$ SR2 $204.02 .02 .02 /$ SR30

$04.02 .03 .02 /$ SR2 $104.02 .03 .02 / S R 22$

$04.02 .03 .03 /$ SR0 $404.02 .04 .01 /$ SR11

$04.02 .04 .02 /$ SR18 $04.02 .05 .02 /$ SR2 2

$04.02 .05 .02 /$ SR32 $04.02 .05 .03 /$ SR2 1

$04.02 .05 .03 /$ SR33 $04.03 .02 .02 /$ SR19

$06.01 .02 /$ SR3 8

\#S $4.14 / 114 / 2$ :

$02.03 .03 /$ SR21 $02.03 .03 /$ SR22 $03.01 /$ SR02 $04.02 .01 .02 /$ SR18

$04.02 .01 .02 /$ SR21 $04.02 .01 .02 /$ SR2 $204.02 .01 .02 /$ SR 30

$04.02 .01 .02 /$ SR41 $04.02 .02 .02 /$ SR18 $04.02 .02 .02 /$ SR2 1

$04.02 .02 .02 /$ SR2 $204.02 .02 .02 /$ SR30 $04.02 .02 .02 /$ SR 41

$04.02 .03 .01 /$ SR55 $04.02 .03 .02 /$ SR18 $04.02 .03 .02 /$ SR2 1

$04.02 .03 .02 /$ SR2 $204.02 .03 .02 /$ SR $3104.02 .03 .02 /$ SR 32

$04.02 .03 .02 /$ SR3 $304.02 .03 .02 /$ SR53 $04.02 .03 .02 /$ SR5 9

$04.02 .04 .01 /$ SR $4504.02 .04 .02 /$ SR2 $6 \quad 04.02 .05 .02 /$ SR 19

$04.02 .05 .02 /$ SR2 $204.02 .05 .02 /$ SR23 $04.02 .05 .02 /$ SR 34

$04.02 .05 .02 /$ SR $4204.02 .05 .03 /$ SR18 $04.02 .05 .03 /$ SR2 1

$04.02 .05 .03 /$ SR2 $204.02 .05 .03 /$ SR3 $304.02 .05 .03 /$ SR 44

$04.02 .05 .04 /$ SR10 $04.02 .05 .04 /$ SR13 $04.02 .05 .04 /$ SR14

$04.03 .02 .02 /$ SR19 $05.01 .02 /$ SR0 $405.03 /$ SR16 $06.01 .02 /$ SR3 8

$06.01 .02 /$ SR39 $06.02 .02 /$ SR0 $506.02 .02 /$ SR13

\#S $5.4 .2 / 138 / 6$ :

$04.02 .03 .01 /$ SR3 6

$04.02 .03 .02 /$ SR 59

$04.02 .03 .01 /$ SR3 $704.02 .03 .02 /$ SR58

$04.02 .03 .02 /$ SR59 $04.02 .03 .03 /$ SR0 6

$04.02 .04 .01 /$ SR2 $704.02 .04 .01 /$ SR29

$04.02 .04 .02 /$ SR 25

$04.02 .04 .02 /$ SR2 $604.02 .04 .02 /$ SR2 6

\#S 5.4.2/140/1:

$04.02 .03 .01 /$ SR59 $04.02 .03 .01 /$ SR60 $04.02 .03 .01 /$ SR61

$04.02 .03 .03 /$ SR0 $804.02 .03 .03 /$ SR09

\#S $5.4 .2 / 142 / 1$ :

$04.02 .04 .01 /$ SR49 $04.02 .04 .01 /$ SR50 $04.02 .04 .01 /$ SR52

\#S $5.12 .1 / 232 / 1$ :

$04.03 .02 .02 /$ SR 15

\#S $5.12 .1 / 232 / 2$ :

$04.02 .01 .02 /$ SR2 6

$04.02 .02 .02 /$ SR2 7

$04.02 .01 .02 /$ SR2 $704.02 .02 .02 /$ SR2 6

$04.02 .03 .02 / S R 28$

$04.02 .03 .02 /$ SR 26

$04.02 .05 .02 /$ SR2 7

$04.02 .03 .02 / S R 27$

$04.02 .05 .03 /$ SR2 $704.02 .05 .04 /$ SR18

$04.02 .05 .03 /$ SR2 6

\#S $6.1 / 306 / 6$ :

$05.03 /$ SR03 $05.03 /$ SR14

\#S $6.1 / 306 / 8$ :

$04.03 .02 .02 /$ SR2 $204.03 .02 .02 /$ SR23 $04.03 .02 .02 /$ SR2 4

$04.03 .02 .02 /$ SR2 $504.03 .02 .02 /$ SR2 6 
\#S $6.1 / 306 / 9$ :

$04.03 .02 .02 /$ SR0 7

\#S $6.1 / 307 / 2$ :

11/SRO1 11/SRO2

\#S $6.1 / 307 / 3$ :

11/SRO1 11/SRO2

\#S $6.2 / 308 / 1$ :

$03.01 /$ SR03 $05.01 .01 /$ SR02

\#S $6.2 / 308 / 2$ :

$05.01 .01 /$ SR04 $05.01 .01 /$ SR0 5

\#S $6.3 / 309 / 1$ :

$02.03 .03 /$ SR21 $02.03 .03 /$ SR2 $203.01 /$ SR02 $04.01 .03 /$ SR0 8

$04.02 .01 .01 /$ SR11 $04.02 .01 .01 /$ SR17 $04.02 .01 .01 /$ SR23

$04.02 .01 .02 /$ SR0 $804.02 .02 .01 /$ SR11 $04.02 .02 .01 /$ SR18

$04.02 .02 .01 /$ SR2 $404.02 .02 .02 /$ SR0 $804.02 .03 .01 / S R 23$

$04.02 .03 .01 /$ SR32 $04.02 .03 .01 /$ SR3 $84.02 .03 .01 /$ SR 44

$04.02 .03 .01 /$ SR50 $04.02 .03 .01 /$ SR62 $04.02 .03 .01 /$ SR70

$04.02 .03 .01 /$ SR77 $04.02 .03 .01 /$ SR82 $04.02 .03 .02 /$ SR0 8

$04.02 .04 .01 /$ SR16 $04.02 .04 .01 /$ SR2 $404.02 .04 .01 /$ SR30

$04.02 .04 .01 /$ SR3 $504.02 .04 .01 /$ SR40 $04.02 .04 .01 /$ SR53

$04.02 .04 .01 /$ SR73 $04.02 .04 .02 /$ SR06 $04.03 .01 /$ SR10 $05.01 .01 /$ SR 10

$05.01 .01 /$ SR11 $05.01 .02 /$ SR0 7

\#S $6.3 / 310 / 1:$

$02.02 .02 /$ SR11 $04.02 .04 .01 /$ SR61 $04.02 .04 .01 /$ SR68 $04.02 .04 .01 /$ SR83

$04.02 .05 .01 /$ SR16 $04.02 .05 .01 /$ SR24 $04.02 .05 .01 /$ SR30

$04.02 .05 .01 /$ SR3 $84.02 .05 .01 /$ SR4 $704.02 .05 .01 /$ SR5 3

$04.02 .05 .01 /$ SR59 $04.02 .05 .01 /$ SR64 $04.02 .05 .01 /$ SR80

$04.02 .05 .01 /$ SR82 $04.02 .05 .02 /$ SR09 $04.02 .05 .03 /$ SR08 $04.03 .03 /$ SR09

$04.03 .04 .01 /$ SR11 $06.01 .02 /$ SR20 $06.01 .02 /$ SR39 $06.01 .02 / S R 43$

\#S $6.3 / 311 / 4:$

$06.01 .02 /$ SR3 8

\#S $6.4 / 313 / 1$ :

$04.03 .04 .01 /$ SR11

\#S $6.5 / 314 / 1$ :

02.01.01/SR08 02.01.01/SR09 02.02.03/SR05 03.01/SR04 03.02/SR01 $03.02 /$ SR02 $05.01 .01 /$ SR01

\#S $6.6 / 316 / 1$ :

06.02.02/SR09 $06.02 .02 /$ SR10 $06.02 .02 /$ SR11 $06.03 /$ SR05 $06.03 /$ SR0 $06.03 /$ SR15

\#S $6.6 / 316 / 4$ :

$04.03 .03 /$ SR10 $04.03 .03 /$ SR $1204.03 .03 /$ SR13 $04.03 .03 /$ SR14

$04.03 .03 /$ SR15 
\#S $6.6 / 317 / 1$ :

$04.02 .01 .02 / \mathrm{SR} 24$

$04.02 .02 .02 /$ SR2 5

$04.02 .04 .02 /$ SR09

$04.02 .05 .03 / S R 24$

$04.02 .05 .04 / S R 16$

$05.02 /$ SR02 $05.02 /$ SR08 $05.02 /$ SR09
$04.02 .01 .02 / S R 25$

$04.02 .03 .02 /$ SR2 4

$04.02 .05 .02 /$ SR2 5

$04.02 .05 .03 / S R 25$

$04.02 .05 .04 /$ SRI 7
$04.02 .02 .02 / S R 24$

$04.02 .03 .02 /$ SR2 5

$04.02 .05 .02 /$ SR2 6

$04.02 .05 .03 /$ SR2 9

$04.03 .02 .02 /$ SR20 $05.02 / S R 01$

\#S $6.6 / 318 / 1$ :

$04.03 .02 .02 /$ SR11 $04.03 .02 .02 /$ SR13 $04.03 .02 .02 /$ SR14

$04.03 .04 .02 /$ SR03 $04.03 .04 .02 /$ SR0 7

\#S $6.7 / 320 / 1$ :

$06.02 .02 /$ SR0 $506.03 /$ SR0 4

\#S $6.7 / 321 / 1$ :

$04.02 .01 .02 /$ SR21 $04.02 .01 .02 / S R 2204.02 .01 .02 / S R 23$

$04.02 .01 .02 /$ SR2 $404.02 .01 .02 /$ SR2 $504.02 .01 .02 /$ SR2 8

$04.02 .01 .02 /$ SR29 $04.02 .01 .02 /$ SR30 $04.02 .01 .02 / S R 40$

$04.02 .01 .02 /$ SR41 $04.02 .02 .02 /$ SR2 $104.02 .02 .02 / S R 22$

$04.02 .02 .02 /$ SR23 $04.02 .02 .02 /$ SR2 $4 \quad 04.02 .02 .02 /$ SR2 5

$04.02 .02 .02 /$ SR2 $804.02 .02 .02 /$ SR29 $04.02 .02 .02 /$ SR30

$04.02 .02 .02 /$ SR $4004.02 .02 .02 /$ SR $4105.02 /$ SR01 $05.02 /$ SR02

\#S $6.7 / 322 / 1$ :

$04.02 .03 .01 /$ SR5 $404.02 .03 .01 /$ SR55 $04.02 .03 .01 /$ SR 57

$04.02 .03 .02 /$ SR21 $04.02 .03 .02 /$ SR2 $204.02 .03 .02 /$ SR2 3

$04.02 .03 .02 /$ SR2 $404.02 .03 .02 /$ SR2 $504.02 .03 .02 /$ SR2 9

$04.02 .03 .02 /$ SR52 $04.02 .03 .02 /$ SR53 $04.02 .03 .02 /$ SR55

$04.02 .04 .01 / S R 4404.02 .04 .01 / S R 4504.02 .04 .01 / S R 47$

$04.02 .04 .02 /$ SR09 $04.02 .05 .01 /$ SR22 $04.02 .05 .01 /$ SR2 3

$04.02 .05 .02 /$ SR22 $04.02 .05 .02 /$ SR23 $04.02 .05 .02 / S R 24$

$04.02 .05 .02 /$ SR2 $504.02 .05 .02 /$ SR2 $604.02 .05 .02 /$ SR2 8

$04.02 .05 .02 /$ SR29 $04.02 .05 .02 /$ SR30 $04.02 .05 .02 /$ SR 31

$04.02 .05 .02 /$ SR42 $04.02 .05 .02 / S R 44 \quad 04.02 .05 .03 /$ SR2 1

$04.02 .05 .03 /$ SR32 $04.02 .05 .03 /$ SR33 $04.02 .05 .03 /$ SR 44

\#S $6.7 / 323 / 1$ :

$02.02 .02 /$ SR11 $04.01 .08 /$ SR04 04.01.08/SR05 $04.01 .08 /$ SR09

$04.01 .08 /$ SR10 $04.02 .05 .03 /$ SR2 $204.02 .05 .03 /$ SR23 $04.02 .05 .03 / S R 24$

$04.02 .05 .03 /$ SR25 $04.02 .05 .03 /$ SR2 $804.02 .05 .03 /$ SR2 9

$04.02 .05 .03 /$ SR43 $04.02 .05 .04 /$ SR14 $04.02 .05 .04 /$ SRI5

$04.02 .05 .04 /$ SR16 $04.02 .05 .04 /$ SR17 $04.03 .02 .02 /$ SR02

$04.03 .02 .02 /$ SR03 $04.03 .02 .02 /$ SR0 $404.03 .02 .02 /$ SR0 7

$04.03 .02 .02 /$ SR10 $04.03 .02 .02 /$ SR12 $04.03 .02 .02 /$ SR13

$04.03 .02 .02 /$ SRI4 $05.03 /$ SR16 $05.03 /$ SR17 $05.03 /$ SRI 8

\#S $6.7 / 324 / 1$ :

$04.02 .01 .02 /$ SR18 $04.02 .02 .02 /$ SR18 $04.02 .03 .02 /$ SR1 8

$04.02 .05 .02 /$ SR19 $04.02 .05 .03 /$ SR18 $04.02 .05 .04 /$ SR09

$04.02 .05 .04 /$ SR10 $04.03 .02 .01 /$ SR0 $806.02 .02 /$ SR13 
APPENDIX J

MODULE CROSS-REFERENCE

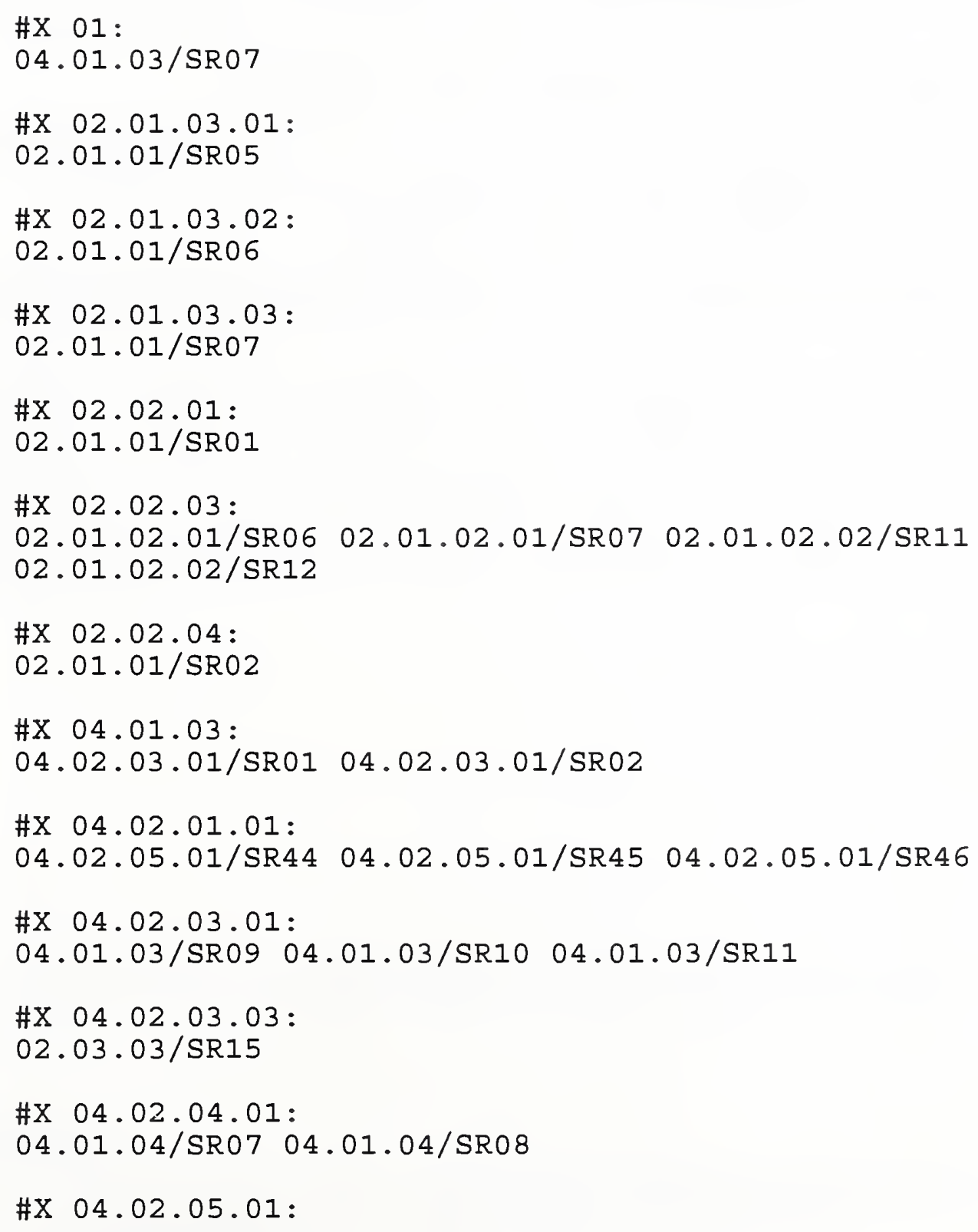




\section{MODULE CROSS-REFERENCE}

$04.02 .05 .04 /$ SRO2

\#X $04.02 .05 .02:$

$04.02 .05 .04 /$ SR13 $04.02 .05 .04 /$ SR14

\#X $04.02 .05 .04:$

$04.02 .05 .01 /$ SR15 $04.02 .05 .01 /$ SR20 $04.02 .05 .01 /$ SR2 1

$04.02 .05 .01 /$ SR75 $04.02 .05 .01 /$ SR76 $04.02 .05 .01 /$ SR7 7

$04.02 .05 .01 /$ SR78 $04.02 .05 .02 /$ SR34 $04.02 .05 .02 / S R 41$

\#X 04.03.04.01:

$02.03 .03 / \mathrm{SR} 20$

\#X $05.01 .02:$

$04.02 .05 .03 /$ SR2 $404.02 .05 .03 /$ SR2 5

\#X $05.02:$

$04.03 .03 /$ SR0 $64.03 .03 /$ SR0 $706.02 .02 /$ SR2 $706.02 .02 /$ SR2 8

$06.03 /$ SR18 $06.03 /$ SR19

\#X $05.03:$

$02.01 .01 /$ SR04 02.01.02.01/SR04 02.01.02.02/SR10 02.01.03.01/SR01

$02.01 .03 .02 /$ SR0 $502.01 .03 .02 /$ SR0 $02.01 .03 .03 /$ SR0 1

\#X $06.01 .02:$

$02.03 .03 /$ SR 10 


\begin{tabular}{|c|c|}
\hline \multirow{3}{*}{$\begin{array}{l}\text { U.S. DEPARTMENT OF COMMERCE } \\
\text { NATIONAL INSTITUTE OF STANDARDS AND TECHNOLOGY }\end{array}$} & $\begin{array}{l}\text { 1. PUBUCATIOM OR REPOAT MUMBEA } \\
\text { N I S T IR } 4953\end{array}$ \\
\hline & 2. PERFOAMIMG OAQANIZATION AEPORT MUMBEA \\
\hline & $\begin{array}{l}\text { 3. PUBLCATION DATE } \\
\text { OCTOBER } 1992\end{array}$ \\
\hline \multicolumn{2}{|l|}{$\begin{array}{l}\text { 4. TITLE AND SUBTITIE } \\
\text { User's Guide for the PHIGS Validation Tests (Version 2) }\end{array}$} \\
\hline \multicolumn{2}{|l|}{$\begin{array}{l}\text { 5. AUTHOR(S) } \\
\text { John V. Cugini, Mary T. Gunn, and Lynne S. Rosenthal }\end{array}$} \\
\hline \multirow{2}{*}{$\begin{array}{l}\text { 6. PERFOALINO ORQANIZATION (IF JOINT OA OTHER THAN MIST, SEE IMSTAUCTIONS) } \\
\text { U.S. DEPARTMENT OF COMMERCE } \\
\text { MATIONAL INSTITUTE OF STANDARDS AND TECHNOLOQY } \\
\text { GAITHEASBUAG, MD 20890 }\end{array}$} & 7. CONTAACT/GRANT NUMBER \\
\hline & 6. TYPE OF REPOAT AND PEAIOD COVEAED \\
\hline
\end{tabular}

9. SPONSORING ORGANIZATION MAME AND COMPLETE ADDAESS (STAEET, CITY, STATE, ZIP)

10. SUPPLEMENTARY NOTES

11. ABSTAACT (A 20O-WOAD OA LESS FACTUAL SUMMAAY OF MOST SIOMIFICANT IMFOAMATION. IF DOCUMENT IMCLUDES A SIONIFICANT BIBLOGRAPHY OA UTERATUAE SUAVEY, MENTION IT HERE,

The PHIGS Validation Tests (PVT), developed by NIST, consist of a large set of Fortran programs which may be used to test how well implementations of PHIGS conform to the standard. The tests are organized into a hierarchical structure of modules which corresponds to the conceptual overview of the standard. The tests are associated with the standard via a set of semantic requirements which are derived directiy from the standard. Cross-reference tables allow the user to find tests relating to specific PHIGS functions and data structures. Directions for installation and operation of the tests are included.

12. KEY WORDS (6 TO 12 ENTAIES; ALPHABETICAL ORDER; CAPITALIE OMLY PROPER MAMES; AMD SEPARATE KEY WORDS BY SEMICOLONS) conformance testing; graphics standards; PHIGS; testing of software; validation of software. 



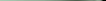

